\title{
HIDDEN ATTRACTORS IN DYNAMICAL SYSTEMS. FROM HIDDEN OSCILLATIONS IN HILBERT-KOLMOGOROV, AIZERMAN, AND KALMAN PROBLEMS TO HIDDEN CHAOTIC ATTRACTOR IN CHUA CIRCUITS
}

\author{
G. A. LEONOV*,‡ and N. V. KUZNETSOV*,† \\ *Department of Applied Cybernetics, \\ Mathematics and Mechanics Faculty, \\ Saint-Petersburg State University, Russia \\ ${ }^{\dagger}$ Department of Mathematical Information Technology, \\ University of Jyväskylä, Finland \\ ${ }^{\dagger}$ nkuznetsov239@gmail.com \\ $\ddagger l e o n o v @ m a t h . s p b u . r u$
}

Received November 21, 2012

From a computational point of view, in nonlinear dynamical systems, attractors can be regarded as self-excited and hidden attractors. Self-excited attractors can be localized numerically by a standard computational procedure, in which after a transient process a trajectory, starting from a point of unstable manifold in a neighborhood of equilibrium, reaches a state of oscillation, therefore one can easily identify it. In contrast, for a hidden attractor, a basin of attraction does not intersect with small neighborhoods of equilibria. While classical attractors are self-excited, attractors can therefore be obtained numerically by the standard computational procedure. For localization of hidden attractors it is necessary to develop special procedures, since there are no similar transient processes leading to such attractors.

At first, the problem of investigating hidden oscillations arose in the second part of Hilbert's 16th problem (1900). The first nontrivial results were obtained in Bautin's works, which were devoted to constructing nested limit cycles in quadratic systems, that showed the necessity of studying hidden oscillations for solving this problem. Later, the problem of analyzing hidden oscillations arose from engineering problems in automatic control. In the 50-60s of the last century, the investigations of widely known Markus-Yamabe's, Aizerman's, and Kalman's conjectures on absolute stability have led to the finding of hidden oscillations in automatic control systems with a unique stable stationary point. In 1961, Gubar revealed a gap in Kapranov's work on phase locked-loops (PLL) and showed the possibility of the existence of hidden oscillations in PLL. At the end of the last century, the difficulties in analyzing hidden oscillations arose in simulations of drilling systems and aircraft's control systems (anti-windup) which caused crashes.

Further investigations on hidden oscillations were greatly encouraged by the present authors' discovery, in 2010 (for the first time), of chaotic hidden attractor in Chua's circuit.

This survey is dedicated to efficient analytical-numerical methods for the study of hidden oscillations. Here, an attempt is made to reflect the current trends in the synthesis of analytical and numerical methods.

\footnotetext{
${ }^{\dagger}$ Address for correspondence
} 
Keywords: Hidden oscillation; hidden attractor; large (normal amplitude) and small limit cycle; Lienard equation; quadratic system; Lyapunov focus values (Lyapunov quantities, PoincaréLyapunov constants, Lyapunov coefficients); 16th Hilbert problem; Aizerman conjecture; Kalman conjecture; absolute stability; nonlinear control system; harmonic balance; describing function method; phase-locked loop (PLL); drilling system; induction motor; Chua circuits.

1. Introduction. Self-Excited and Hidden Oscillations . . . . . . . . . . . . . . . . . . 3

2. 16th Hilbert's Problem . . . . . . . . . . . . . . . . . . . . . . . . . . . . . . . . 7

2.1. Limit cycles of two-dimensional quadratic systems. Hilbert-Kolmogorov's

problem . . . . . . . . . . . . . . . . . . . . . 7

2.2. Quadratic systems reduction . . . . . . . . . . . . . . . . . . . . 8

2.3. Transformation of two-dimensional quadratic system to discontinuous

Lienard system . . . . . . . . . . . . . . . . . . . . . . . . . . . . . . . . . 9

2.4. Asymptotic integration method for discontinuous Lienard equation . . . . . . . . 9

2.5. Global analysis: Boundedness of solutions, existence of one and two limit cycles . . . . . . . . . . . . . . . . . . . . . . . . . . 13

2.6. Visualization of limit cycles in quadratic system . . . . . . . . . . . . . . . . . 14

2.7. Local analysis: Computation of Lyapunov values and small limit cycles . . . . . . 16

2.7.1. Lyapunov values definition . . . . . . . . . . . . . . . . . . . . . . . 16

2.7.2. Direct method for computation of Lyapunov values in Euclidean coordinates and in the time domain . . . . . . . . . . . . . . . . . . . . 18

2.7.3. Poincaré method based on Lyapunov function construction . . . . . . . . 20

2.7.4. Lyapunov values of Lienard system . . . . . . . . . . . . . . . . . . . . 21

2.7.5. Lyapunov values and small limit cycles in quadratic systems . . . . . . . . 22

2.8. Large and small limit cycles . . . . . . . . . . . . . . . . . . . . . . . . . . . 23

2.9. Nonlocal theory on the existence of nested large limit cycles

in quadratic system . . . . . . . . . . . . . . . . . . . . . . . . . . . . 24

2.10. Solution of Kolmogorov's problem. Visualization of four limit cycles . . . . . . . 28

3. Hidden Oscillations in Applied Models . . . . . . . . . . . . . . . . . . . . . . . . 28

3.1. Phase-locked-loop circuits . . . . . . . . . . . . . . . . . . . . . . . . . . . . 28

3.2. Electrical machines . . . . . . . . . . . . . . . . . . . . . . . . . . . . . . 32

3.2.1. Two-mass mathematical model of drilling system . . . . . . . . . . . . . 32

3.2.2. Mathematical model of drilling system actuated by induction motor . . . . 33

4. Aizerman's and Kalman's Conjectures on Absolute Stability of Control System . . . . 34

4.1. Analytical-numerical procedure for hidden attractors localization . . . . . . . . . 36

4.2. Small parameter and describing function method . . . . . . . . . . . . . . . . 37

4.3. Describing function method justification . . . . . . . . . . . . . . . . . . . . . 38

4.3.1. System reduction . . . . . . . . . . . . . . . . . . . . . . . . . . . . . 38

4.3.2. Poincaré map for harmonic linearization in the noncritical case . . . . . . 38

4.3.3. Poincaré map for harmonic linearization in the critical case . . . . . . . . 42

4.4. Hidden oscillations in counterexamples to Aizerman's and

Kalman's conjectures . . . . . . . . . . . . . . . . . . . . . . . . . . . . . . 47

5. Hidden Attractor in Chua's Circuits . . . . . . . . . . . . . . . . . . . . . . . . . 60

6. Conclusions . . . . . . . . . . . . . . . . . . . . . . . . . . 62

Acknowledgments . . . . . . . . . . . . . . . . . . . . . . . . . . 62

References .. . . . . . . . . . . . . . . . . . . . . . 63 


\section{Introduction. Self-Excited and Hidden Oscillations}

In the first half of the last century during the initial period of the development of the theory of nonlinear oscillations [Timoshenko, 1928; Krylov, 1936; Andronov et al., 1966; Stoker, 1950], much attention was given to the analysis and synthesis of oscillating systems, for which the problem of the existence of oscillations can be solved with relative ease. These investigations were encouraged by the applied research on periodic oscillations in mechanics, electronics, chemistry, biology and so on (see, e.g. [Andronov et al., 1966; Strogatz, 1994]; (at the end of 19th century, this research was begun in Rayleigh's works devoted to the study of string oscillations in musical instruments [Rayleigh, 1877]). The structure of many applied systems considered was such that the existence of oscillations was "almost obvious" - the oscillation was excited from an unstable equilibrium (so called selfexcited oscillation). From a computational point of view this allows one to use a standard computational procedure, in which after a transient process, a trajectory, starting from a point of unstable manifold in a neighborhood of equilibrium, reaches a state of oscillation, therefore one can easily identify it.

Later, in the middle of 20th century, in applied systems except for self-excited periodic oscillations, numerically chaotic oscillations [Ueda et al., 1973; Lorenz, 1963] were found to be also excited from an unstable equilibrium and can be computed by the standard computational procedure. Nowadays, thousands of publications have been devoted to the computation and analysis of self-excited chaotic oscillations.

Note that for the computation of oscillations by the standard computational procedure, it is necessary that the oscillation has an attraction domain. By such property of domain, this computational procedure can reach the oscillation and identify it. An attracting oscillation and an attracting set of oscillations below will be called an attractor. Here the ideology of transient process is transferred naturally from the control theory into computational mathematics and the computational process of selfexcited attractors.

A further study showed that the self-excited periodic and chaotic oscillations did not give exhaustive information about the possible types of oscillations. In the middle of 20th century, the examples of periodic and chaotic oscillations of another type were found, later called [Leonov et al., 2011c] hidden oscillations and hidden attractors, of which the basin of attraction does not intersect with small neighborhoods of equilibria. Numerical localization, computation, and analytical investigation of hidden attractors are much more challenging problems, since here there is no possibility to use information about equilibria for organization of similar transient processes in the standard computational procedure. Thus, the hidden attractors cannot be computed by using this standard procedure. Furthermore, in this case it is unlikely that the integration of trajectories with random initial data furnishes hidden attractor localization since a basin of attraction can be very small and the dimension of hidden attractor itself can be much less than the dimension of the considered system.

At first, the problem of analyzing hidden oscillations arose in the second part of Hilbert's 16th problem (1900) for two-dimensional polynomial systems [Hilbert, 1901-1902]. The first nontrivial results were obtained in Bautin's works [Bautin, 1939, 1949, 1952], which were devoted to constructing nested limit cycles in quadratic systems and showed the necessity of studying hidden oscillations for solving this problem.

Later, the problem of analyzing hidden oscillations arose from engineering problems in automatic control. In the middle of the last century, Kapranov studied [Kapranov, 1956] qualitative behavior of PLL systems, widely used nowadays in telecommunications and computer architectures, and estimated stability domains. In these investigations, Kapranov assumed that in PLL systems there were self-excited oscillations only. However, in 1961, Gubar' [1961] revealed a gap in Kapranov's work and showed analytically the possibility of the existence of hidden oscillations in two-dimensional system of phase-locked loop: thus, from a computational point of view, the system considered was globally stable (all the trajectories tend to equilibria), but, in fact, there was a bounded domain of attraction only.

In 1950-60's the investigations of widely known Markus-Yamabe [1960], Aizerman [1949], and Kalman [1957] conjectures on absolute stability have led to the finding of hidden oscillations in automatic control systems with a unique stable stationary point and with a nonlinearity, which belongs 
to the sector of linear stability (see, e.g. [Krasovsky, 1952; Pliss, 1958; Fitts, 1966; Bernat \& Llibre, 1996; Bragin et al., 2011; Leonov \& Kuznetsov, 2013a]).

At the end of the last century the difficulties of numerical analysis of hidden oscillations arose [Lauvdal et al., 1997] in simulation of aircraft's control systems (anti-windup scheme) and caused aircraft crashes.

In the second half of the twentieth century, the problems considered stimulated a large number of various investigations. Hilbert's sixteenth problem stimulated the development of bifurcation theory and the theory of normal forms and Aizerman problem stimulated the development of the theory of absolute stability. The most complete bibliography is available in [Reyn, 1994; Chavarriga \& Grau, 2003; Li, 2003; Liberzon, 2006], involving more than two thousand references.

Further investigations of hidden oscillations were greatly encouraged by the authors' discovery, in 2010 (for the first time), of chaotic hidden attractor in generalized Chua's circuit [Kuznetsov et al., 2010; Leonov et al., 2010c] and later discovery of chaotic hidden attractor in classical Chua's circuit [Leonov et al., 2011c]. It should be remarked that for the last thirty years, several thousand publications, in which a few hundreds of attractors were discussed, have been devoted to Chua's circuit and its various modifications. However, up to now these Chua's attractors were self-excited.

The present survey is dedicated to some efficient analytical-numerical methods for the study of oscillations. Here, an attempt is made to reflect the current trends in synthesis of analytical and numerical methods.

The analytical methods considered are focused on the creation of constructive computational algorithms and applying the powerful computer techniques to solve complex mathematical problems. Here, following Poincaré's advice "to construct the curves defined by differential equations" [Poincaré, 1881], which after the appearance of modern computers became even more actual and assumed a new sense, the main attention is focused on the development of constructive methods for scientific visualization [Earnshaw \& Wiseman, 1992] "to gain understanding and insight into the data" and "to promote a deeper level of understanding of the data under investigation and to foster new insight into the underlying processes".
With this aim, in this work, new approaches are developed.

- The method of asymptotic integration of Lienard equation. This method is based on substantial extension to the classical method, in which smooth mappings of phase plane on the Poincaré sphere are used. Here, we use various classes of such mappings, each of which acts on a separate part of the phase plane. Such an approach permits one to obtain new results, by far smaller number of analytical formulas, and preserve geometric visualization, and explains the main steps of the proof by a few pictures.

- The modification of harmonic linearization and describing function methods for the critical case (when the generalized Routh-Hurwitz conditions are satisfied). In engineering practice, for the analysis of the existence of periodic solutions, classical harmonic linearization and describing function methods are widely used. However, these classical methods are not strictly mathematically reasonable and can lead to incorrect results (e.g. as for the critical case in Aizerman's and Kalman's conjectures). The special modification of these methods, based on the method of small parameter, permits one to obtain the strict justification of the existence of periodic solution and to define the initial data of this solution.

- The effective computational procedures of attractors' localization. The harmonic linearization method, the classical method of small parameter, and numerical methods together allow one to perform the localization of an attractor by a multistep procedure with the use of harmonic linearization method at the first step. The proposed procedure, based on the continuation principle, permits one to follow numerically from the transformation of a starting periodic solution, defined analytically, to a periodic solution or chaotic attractor. Here, it is important for a local attraction domain of the considered solution to be preserved.

Consider classical examples of visualization of self-excited oscillations.

Example 1.1 [Rayleigh's string oscillator]. In studying string oscillations Rayleigh [1877] discovered first that in two-dimensional nonlinear dynamical system can arise undamped vibrations without external periodic action (limit cycles). Consider the 


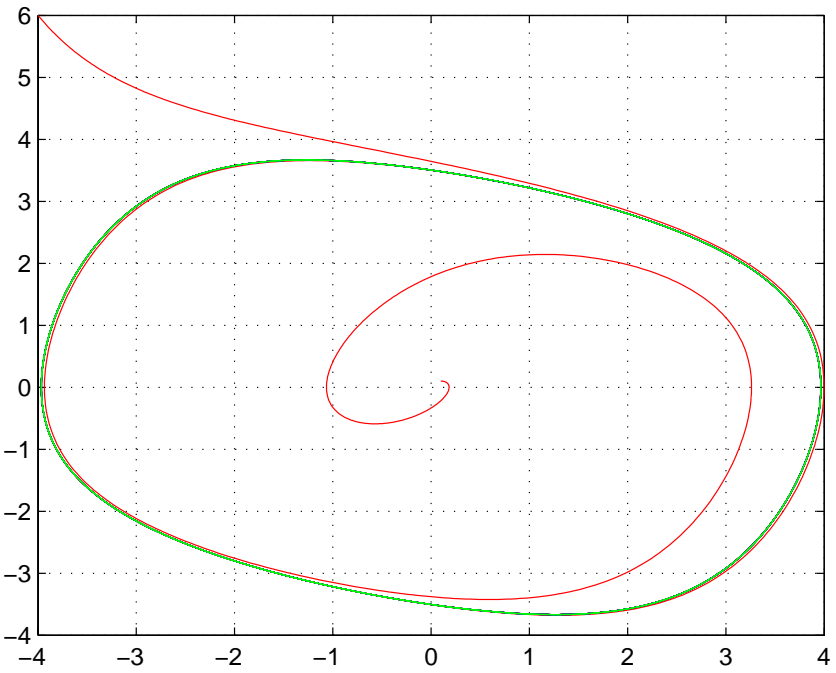

Fig. 1. Localization of limit cycle in Rayleigh system.

localization of limit cycle in Rayleigh system

$$
\ddot{x}-\left(a-b \dot{x}^{2}\right) \dot{x}+x=0,
$$

for $a=1, b=0.1$. In Fig. 1, a limit cycle is localized by two trajectories (each trajectory begins in red, and ends in green), attracting to the limit cycle.

The extension of Eq. (1) is a well-known van der Pol equation.

Example 1.2 [Van der Pol oscillator]. Consider oscillations arising in an electrical circuit - the van der Pol oscillator [van der Pol, 1926]:

$$
\ddot{x}+\mu\left(x^{2}-1\right) \dot{x}+x=0,
$$

and make a computer simulation for the parameter $\mu=2$ (see Fig. 2).

Example 1.3 [Belousov-Zhabotinsky (BZ) reaction]. In 1951, Belousov discovered first oscillations in chemical reactions in a liquid phase [Belousov, 1959]. Consider one of the Belousov-Zhabotinsky dynamic models

$$
\begin{aligned}
\varepsilon \dot{x} & =x(1-x)+\frac{f(q-x)}{q+x} z \\
\dot{z} & =x-z,
\end{aligned}
$$

and fulfill a computer simulation with standard parameters $f=2 / 3, q=8 \times 10^{-4}, \varepsilon=4 \times 10^{-2}$.

Figure 3 shows the effect of stiffness of the system (an abrupt change in the direction of

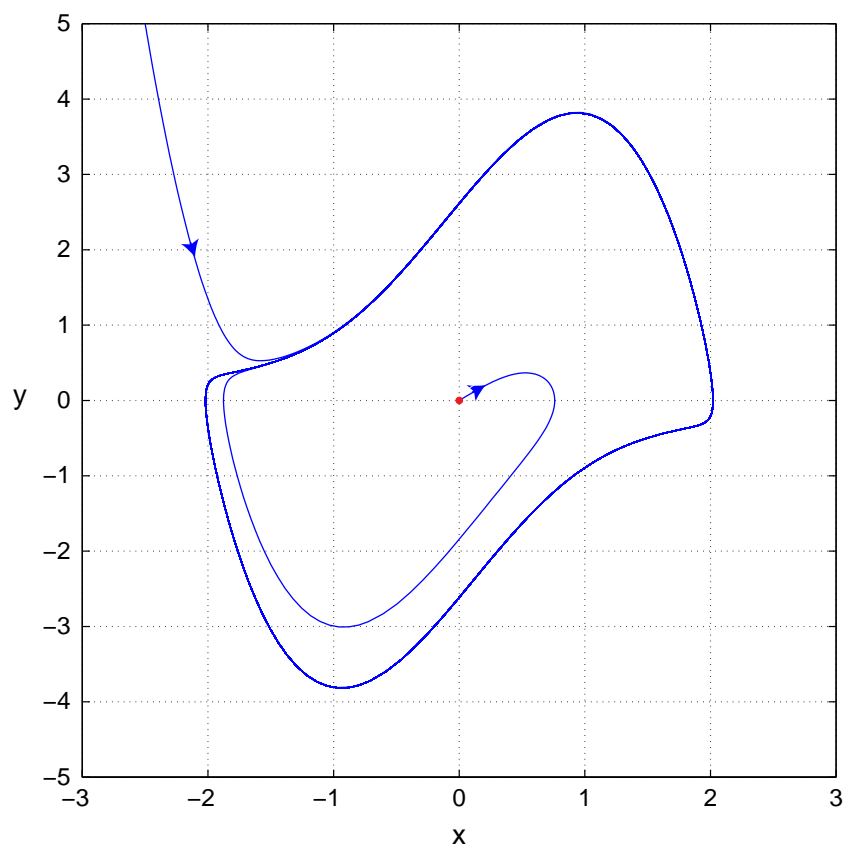

Fig. 2. Numerical localization of limit cycle in van der Pol oscillator.

trajectories), which substantially complicates numerical analysis of such systems [Hairer \& Wanner, 1991].

Now consider classical three-dimensional dynamic models, where unlike in two-dimensional systems, except for the periodic ones the chaotic oscillations can arise.

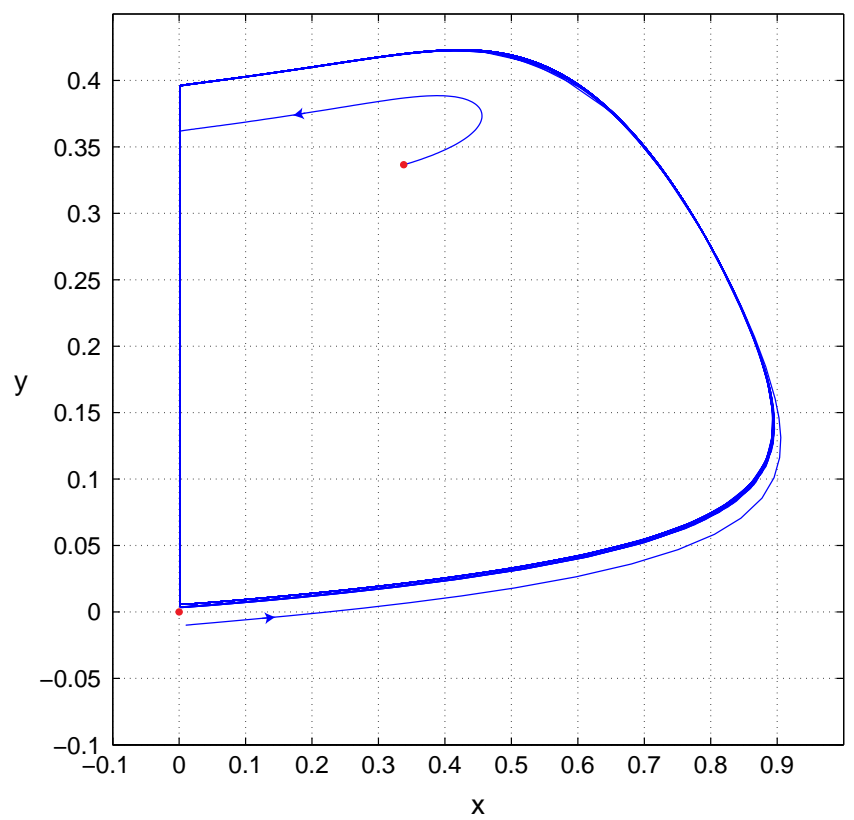

Fig. 3. Numerical localization of limit cycle in BelousovZhabotinsky (BZ) reaction. 


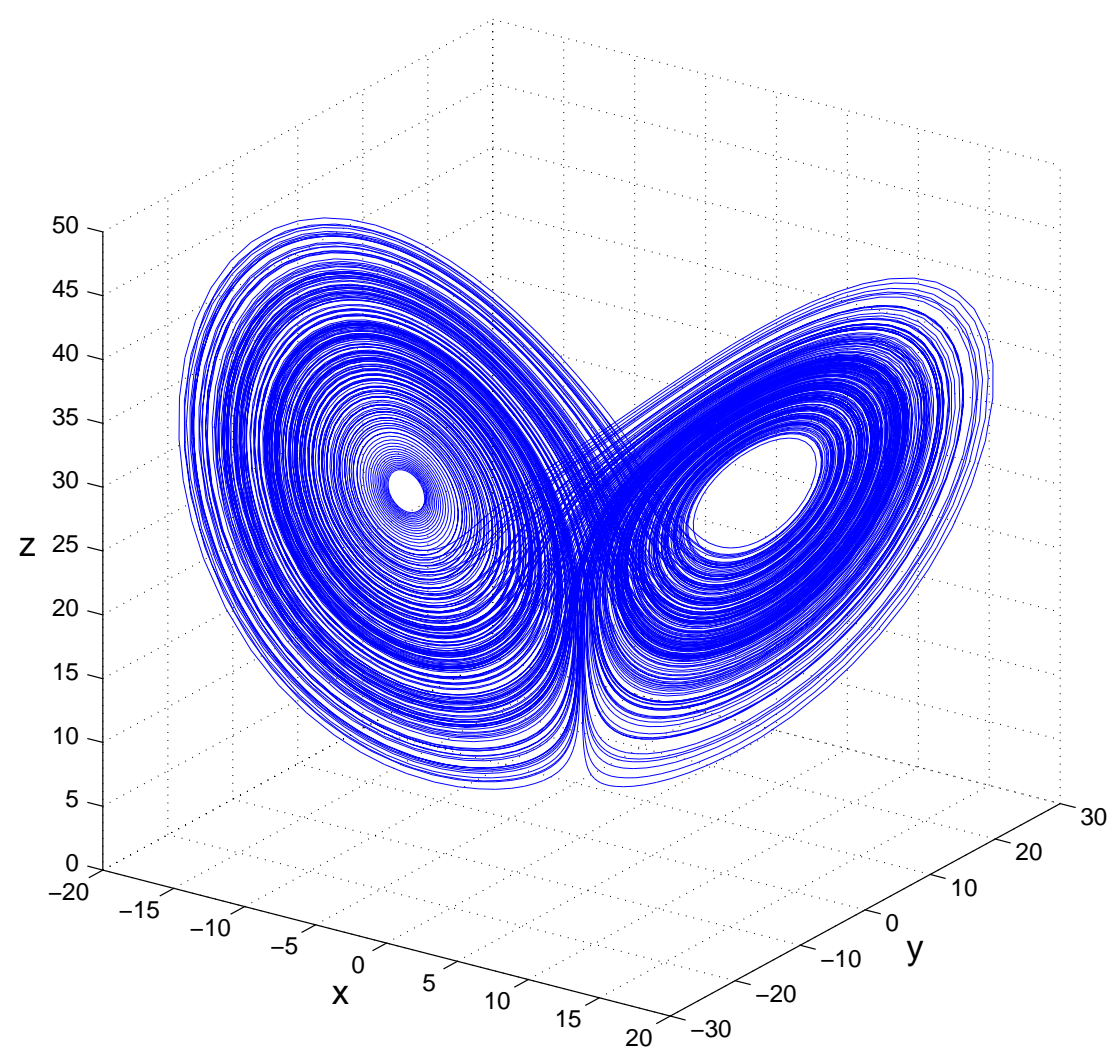

Fig. 4. Numerical localization of chaotic attractor in Lorenz system.

Example 1.4 [Lorenz system]. Consider Lorenz system [Lorenz, 1963]

$$
\begin{aligned}
& \dot{x}=\sigma(y-x), \\
& \dot{y}=x(\rho-z)-y, \\
& \dot{z}=x y-\beta z,
\end{aligned}
$$

and make its simulation with standard parameters $\sigma=10, \beta=8 / 3, \rho=28$ (see Fig. 4).

Example 1.5 [Chua system]. Consider the behavior of classical Chua circuit [Chua, 1992].

In the dimensionless coordinates a dynamic model of this circuit is as follows

$$
\begin{aligned}
& \dot{x}=\alpha(y-x)-\alpha f(x), \\
& \dot{y}=x-y+z, \\
& \dot{z}=-(\beta y+\gamma z) .
\end{aligned}
$$

Here the function

$$
\begin{aligned}
f(x) & =m_{1} x+\left(m_{0}-m_{1}\right) \operatorname{sat}(x) \\
& =m_{1} x+\frac{1}{2}\left(m_{0}-m_{1}\right)(|x+1|-|x-1|)
\end{aligned}
$$

characterizes a nonlinear element, of the system, called Chua's diode; $\alpha, \beta, \gamma, m_{0}, m_{1}$ are parameters of the system. In this system, the strange attractors discovered [Matsumoto, 1990] were called then Chua's attractors. To date all the known classical Chua's attractors are excited from an unstable equilibria. This makes it possible to compute various Chua's attractors [Bilotta \& Pantano, 2008] with relative ease. For simulation of this system, we use the following parameters $\alpha=9.35, \beta=14.79$, $\gamma=0.016, m_{0}=-1.1384, m_{1}=0.7225$ (see Fig. 5).

For all the above examples, the limit cycles and attractors are excited from an unstable equilibrium. From a computational point of view, in this case, it is possible to use a numerical method in which after a transient process, a trajectory, starting from a point of unstable manifold in a neighborhood of unstable equilibrium, reaches a state of attractor, and therefore it can be easily identified.

This series of examples can be extended to the cases of other well-known systems (see [Timoshenko, 1928; Andronov et al., 1966; Stoker, 1950; Chance et al., 1973; Strogatz, 1994; Jones et al., 2010] and others). We now consider problems where hidden oscillations occur. 


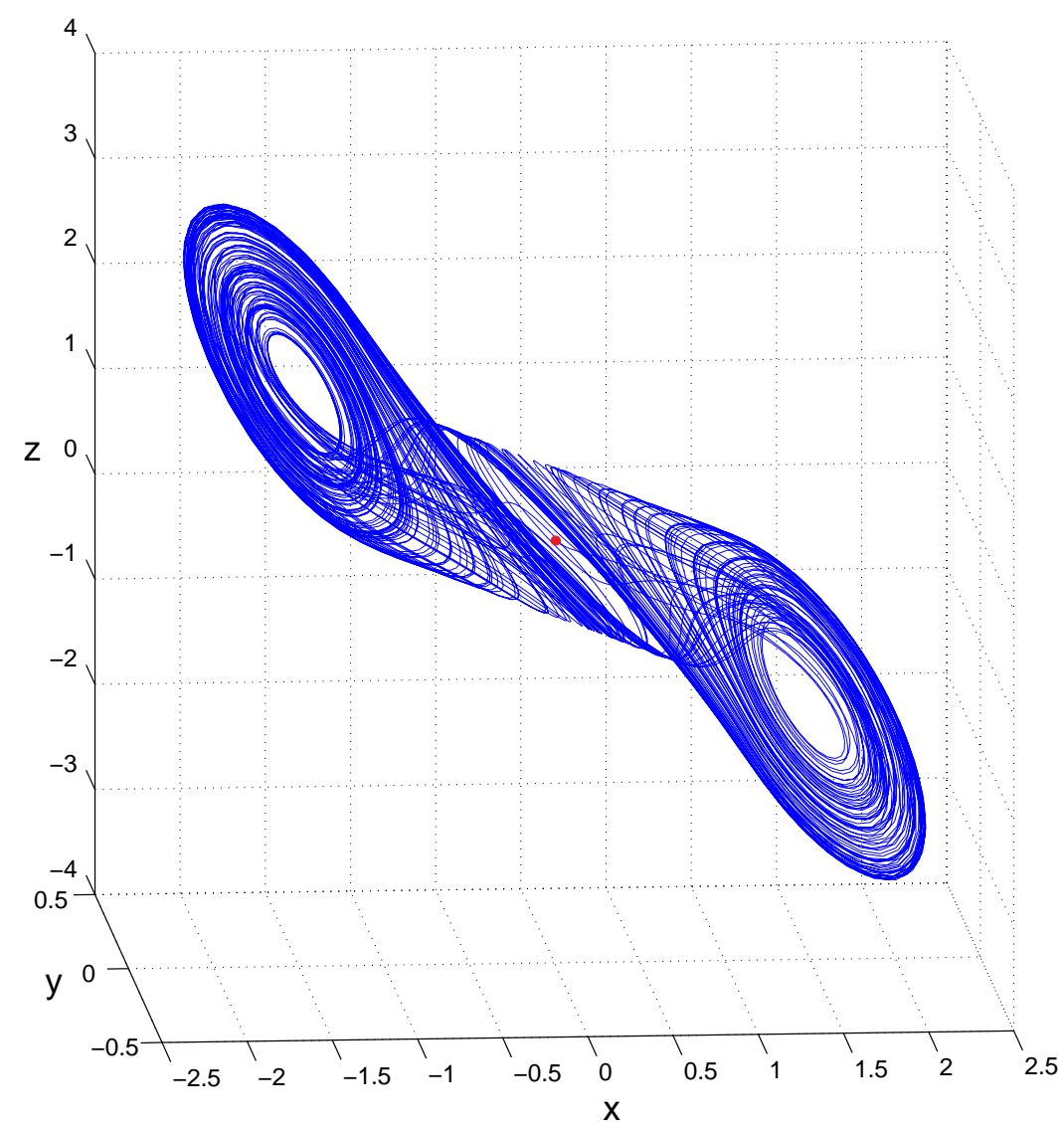

Fig. 5. The numerical localization of chaotic attractor in Chua's circuit.

\section{16th Hilbert's Problem}

\subsection{Limit cycles of two-dimensional quadratic systems. Hilbert-Kolmogorov's problem}

In 1900, David Hilbert posed the problem to investigate the number and possible dispositions of limit cycles in two-dimensional polynomial systems in relation to the degree of the considered polynomials.

... This is the question as to the maximum number and position of Poincaré's boundary cycles (cycles limits) for a differential equation of the first order and degree of the form $d x / d y=Y / X$, where $X$ and $Y$ are rational integral functions of the $n$th degree in $x$ and $y . .$.

This is the second part of 16th Hilbert Problem [Hilbert, 1901-1902], which Smale [1998] reformulated later in the following way: Is there a bound $K=H(n)$ on the number of limit cycles of the form $K<n^{q}$ for the polynomial system

$$
\frac{d x}{d t}=P_{n}(x, y), \quad \frac{d y}{d t}=Q_{n}(x, y),
$$

where $n$ is the maximum of the degree of polynomials $P_{n}$ and $Q_{n}$ and $q$ is a universal constant.

For more than a century, in attempting to solve this problem, numerous analytical results were obtained (see, references in [Reyn, 1994]). But the problem is still far from being resolved even for a simple class of quadratic systems.

The important direction of the study of Hilbert's sixteenth problem is a proof of finiteness of the number of limit cycles. The history of this proof for polynomial systems on a plane is connected with the well-known work of Dulac [1923]. However, later in Dulac's proof, gaps were found [Ilyashenko, 1985]. These gaps were corrected by Bamon [1985] (for quadratic polynomial systems) and, independently, by Ilyashenko [1991] and by Ecalle [1992] (for general polynomial systems).

The creation of effective methods for the construction of systems with limit cycles was initiated by Bautin $[1949,1952]$. In his works, for the construction of nested limit cycles, an effective analytical method was proposed based on determining the sequential symbolic expressions of Lyapunov values 
(called also focus values, Lyapunov quantities, Lyapunov coefficients, Poincaré-Lyapunov constants). The sequential computation of Lyapunov values, used by Bautin, allowed him to discriminate first a class of quadratic systems, in which three nested limit cycles (here inner cycle is obviously a hidden oscillation) can be found in the neighborhood of degenerate focus by small perturbations of system coefficients [Bautin, 1952] (such cycles are naturally called small or small-amplitude limit cycles). Next, Petrovskii and Landis [1955] asserted that quadratic system can have less than or equal to three limit cycles. But later they reported a gap in the proof [Petrovskii \& Landis, 1959]. Quadratic systems were found with four limit cycles [Chen \& Wang, 1979; Shi, 1980] (three nested small limit cycles, obtained by Bautin's technique, and one large (or normalamplitude) limit cycle, surrounding another focus equilibrium).

So, up to now, the best result of possible estimation for number $H(2)$ of limit cycles in quadratic system is $H(2) \geq 4$ and it is finite (for cubic systems $H(3) \geq 13$ [Li et al., 2009], and in the work [Han \& Li, 2012] a lower estimate is given for the Hilbert number $H(n)$ : it grows at least as rapidly as $(2 \ln 2)^{-1}(n+2)^{2} \ln (n+2)$ for all large $\left.n\right)$.

The appearance of modern computers permits one to use numerical simulation of complicated nonlinear dynamical systems and to obtain new information on a structure of their trajectories. However, the possibilities of "simple" approach, based on the construction of trajectories by numerical integration of the considered differential equations, turned out to be highly limited.

In studying the 16th Hilbert problem, the numerical search and the construction of limit cycles are a rather complicated problem by reason of the presence of nested limit cycles (which, as a rule, are constructed analytically with the use of small perturbations and bifurcation analysis), the effects of trajectory rigidity [Leonov et al., 2011a], and a large dimension of the considered space of system's parameters. The latter was shown, for example, in the task posed by academician Kolmogorov and described by Arnold in [Arnold, 2005]: To estimate the number of limit cycles of square vector fields on plane, Kolmogorov had distributed several hundreds of such fields among a few hundreds of students of Mechanics and Mathematics Faculty of Moscow State University as a mathematical practice. Each student had to find the number of limit cycles of a field. The result of this experiment was absolutely unexpected: not a single field had a limit cycle! A limit cycle is conserved when the field coefficients are slightly changed. Hence, systems with one, two, three (and even, as would become known later, four) limit cycles form open sets in a space of coefficients such that in the case of a random choice of polynomial coefficients the probabilities of entering into these sets are positive. The fact that this did not happen suggests that the above-mentioned probabilities are obviously small.

The result of this experiment also demonstrates the need to develop purposeful methods to search periodic oscillations, that is, both analytical and numerical methods with the use of the full power of current computational techniques.

Here, we are concerned with the Kolmogorov problem and will elucidate whether twodimensional quadratic dynamical systems exist for which the students might have revealed and visualize limit cycles in their tutorial exercise described above.

\subsection{Quadratic systems reduction}

A two-dimensional quadratic system may be written as

$$
\begin{aligned}
& \dot{x}=a_{1} x^{2}+b_{1} x y+c_{1} y^{2}+\alpha_{1} x+\beta_{1} y, \\
& \dot{y}=a_{2} x^{2}+b_{2} x y+c_{2} y^{2}+\alpha_{2} x+\beta_{2} y,
\end{aligned}
$$

where $a_{j}, b_{j}, c_{j}, \alpha_{j}, \beta_{j}$ are real numbers.

System (8) can be reduced to a more convenient form. For this purpose, the following simple assertions will be proved.

Proposition 1. Without loss of generality, it can be assumed that $c_{1}=0$.

For the proof, the linear change $x \rightarrow x+\nu y$, $y \rightarrow y$ is made. Here $\nu$ is a real solution of the following equation

$$
-a_{2} \nu^{3}+\left(a_{1}-b_{2}\right) \nu^{2}+\left(b_{1}-c_{2}\right) \nu+c_{1}=0 .
$$

The equation always has a real solution if $a_{2} \neq 0$.

If $a_{2}=0$, then after the change of variables $\{x, y\} \rightarrow\{y, x\}$ it can be obtained that $c_{1}=0$ and Proposition 1 is proved.

Proposition 2. Suppose, $c_{1}=0, \beta_{1} \neq 0$. Then, without loss of generality, it can be assumed that $\alpha_{1}=0$.

The proof of this assertion is based on the use of the following linear change $x \rightarrow x, y \rightarrow y-\alpha_{1} x / \beta_{1}$. 
Proposition 3. Let $c_{1}=0, \alpha_{1}=0, a_{1} \neq 0, b_{1} \neq 0$, $\beta_{1} \neq 0$. Then, without loss of generality, it can be assumed that

$$
c_{1}=\alpha_{1}=0, \quad a_{1}=b_{1}=\beta_{1}=1 .
$$

The proof is by the following linear change

$$
x \rightarrow \frac{\beta_{1}}{b_{1}} x, \quad y \rightarrow \frac{a_{1} \beta_{1}}{b_{1}^{2}} y, \quad t \rightarrow \frac{b_{1}}{a_{1} \beta_{1}} t .
$$

By Propositions 1-3, it can be assumed that

$$
c_{1}=\alpha_{1}=0, \quad a_{1}=b_{1}=\beta_{1}=1 .
$$

Then in the place of system (8), we can consider

$$
\begin{aligned}
& \dot{x}=x^{2}+x y+y, \\
& \dot{y}=a_{2} x^{2}+b_{2} x y+c_{2} y^{2}+\alpha_{2} x+\beta_{2} y .
\end{aligned}
$$

Further, the indices of coefficients of system (11) will be omitted.

Other widely used reductions of quadratic systems can be found in [Ye et al., 1986].

\subsection{Transformation of two-dimensional quadratic system to discontinuous Lienard system}

Here it will be considered, the nonlinear transformation of quadratic system to Lienard system (or Lienard equation), which in many cases allows to effectively investigate limit cycles (see, e.g. [Lynch, 2010; Borodzik \& Żołądek, 2008; de Maesschalck \& Dumortier, 2011; Yang \& Han, 2012]).

Consider a quadratic system, and reduce it to special Lienard equation. The transformation of quadratic systems to Lienard equation can be found in [Cherkas \& Zhilevich, 1970; Ye et al., 1986; Coppel, 1989, 1991; Albarakati et al., 2000]. Here, the authors follow the works [Leonov, 1997, 1998, 2006].

At first, prove the following

Proposition 4. The half-plane

$$
\Gamma_{x>-1}=\left\{x>-1, y \in \mathbb{R}^{1}\right\}
$$

is positively invariant with respect to system (11).

The assertion follows from the fact that $\dot{x}(t)=$ $x(t)^{2}=1$ for $x(t)=-1$.

System (11) can be reduced to the Lienard system

$$
\dot{x}=u, \quad \dot{u}=-f(x) u-g(x)
$$

by the change of variables

$$
\left(y+\frac{x^{2}}{x+1}\right)|x+1|^{q} \rightarrow u, \quad x \rightarrow x .
$$

Here

$$
\begin{aligned}
f(x)= & \Psi(x)|x+1|^{q-2}, \\
\Psi(x)= & (2 c-b-1) x^{2}-(2+b+\beta) x-\beta, \\
g(x)= & \Phi(x) \frac{|x+1|^{2 q}}{(x+1)^{3}}, \\
\Phi(x)= & -x(x+1)^{2}(a x+\alpha) \\
& +x^{2}(x+1)(b x+\beta)-c x^{4}
\end{aligned}
$$

and $q=-c$.

By the transformation reverse to (13), system (12) takes the form

$$
\begin{aligned}
& \dot{x}=\left(x^{2}+x y+y\right) \frac{|x+1|^{q}}{(x+1)}, \\
& \dot{y}=\left(a x^{2}+b x y+c y^{2}+\alpha x+\beta y\right) \frac{|x+1|^{q}}{(x+1)} .
\end{aligned}
$$

By Proposition 4, the trajectories of this system are also the trajectories of system (11) on the left and right of line $\{x=-1\}$.

Further, a method of asymptotic integration is described [Leonov, 2010a], which permits one to obtain a rather simple existence criteria of limit cycles of system (12) and the corresponding quadratic system.

\subsection{Asymptotic integration method for discontinuous Lienard equation}

Classical analysis of nonlocal qualitative behavior of two-dimensional systems is based on the use of various smooth mappings, of the whole phase space on a sphere, under which the infinitely distant points are mapped on a great circle (Poincaré compactification, Poincaré-Lyapunov compactification) (see, e.g. [Bautin \& Leontovich, 1976; Dumortier et al., 2006]). This allows to consider a system behavior on the closed disk. The analysis of behavior of critical points at infinity (by special changes, infinite points are mapped into finite ones and a standard local analysis can be applied), the local analysis of critical points, and the analysis of invariants permit one to consider the appearance of limit cycles. 
Consider another effective approach for investigation of two-dimensional quadratic systems. This approach is based on the extension of the classical method, in which smooth mappings of phase plane on the Poincaré sphere are applied and in this case, various classes of such maps are used, each of which acts on a separate part of phase plane. Such an approach permits one to obtain new results by far smaller number of analytical formulas and to preserve geometric visualization, so the main steps of the proof are explained by a few figures. Further, this approach will be applied to quadratic systems. It can also be extended to other classes of two-dimensional systems.

Here, a main scheme of applying the method of asymptotic integration to system (12) is presented. For the description of this method, Lienard system (12) with functions (14), where $q \in(-1,0)$, is considered.

Suppose that for large $|x|$ the following relations

$$
\begin{gathered}
\frac{\Psi(x)}{x^{2}}=A+O\left(\frac{1}{|x|}\right), \quad \frac{\Phi(x)}{x^{4}}=B+O\left(\frac{1}{|x|}\right), \\
\Psi(-1)=P, \quad \Phi(-1)=Q
\end{gathered}
$$

are satisfied. It is well known that system (12) is equivalent to the first order equation

$$
F \frac{d F}{d x}+f(x) F+g(x)=0 .
$$

Introduce the following transformations of Eq. (16):

$$
\begin{array}{ll}
\text { (1) } & z=(x+1)^{q+1}:\{x \geq 0\} \rightarrow\{z \geq 1\}, G(z)= \\
& F\left(z^{\frac{1}{q+1}}-1\right), \\
\text { (2) } & z=(x+1)^{q-1}:\{x \in(-1,0]\} \rightarrow\{z \geq 1\}, G(z)= \\
& F\left(z^{\frac{1}{q-1}}-1\right), \\
\text { (3) } & z=|x+1|^{q+1}:\{x \leq-2\} \rightarrow\{z \geq 1\}, G(z)= \\
& F\left(-z^{\frac{1}{q+1}}-1\right), \\
\text { (4) } & z=|x+1|^{q-1}:\{x \in[-2,-1)\} \rightarrow\{z \geq 1\}, \\
& G(z)=F\left(-z^{\frac{1}{q-1}}-1\right) .
\end{array}
$$

In these cases Eq. (16) can be transformed in the following way:

$$
\begin{aligned}
G d G & +\frac{1}{(q+1)} \frac{\Psi\left(z^{\frac{1}{q+1}}-1\right)}{z^{\frac{2}{q+1}}} G d z \\
& +\frac{1}{(q+1)} \frac{\Phi\left(z^{\frac{1}{q+1}}-1\right)}{z^{\frac{4}{q+1}}} z d z=0
\end{aligned}
$$

(2)

$$
\begin{aligned}
G d G & +\frac{1}{(q-1)} \Psi\left(z^{\frac{1}{q-1}}-1\right) G d z \\
& +\frac{1}{(q-1)} \Phi\left(z^{\frac{1}{q-1}}-1\right) z d z=0
\end{aligned}
$$

(3)

$$
\begin{aligned}
G d G & -\frac{1}{(q+1)} \frac{\Psi\left(z^{\frac{1}{q+1}}-1\right)}{z^{\frac{2}{q+1}}} G d z \\
& +\frac{1}{(q+1)} \frac{\Phi\left(z^{\frac{1}{q+1}}-1\right)}{z^{\frac{4}{q+1}}} z d z=0,
\end{aligned}
$$

(4)

$$
\begin{aligned}
G d G & -\frac{1}{(q-1)} \Psi\left(z^{\frac{1}{q-1}}-1\right) G d z \\
& +\frac{1}{(q-1)} \Phi\left(z^{\frac{1}{q-1}}-1\right) z d z=0 .
\end{aligned}
$$

For the large $z$, Eq. (17) is close to the equation

$$
G d G+\frac{A}{(q+1)} G d z+\frac{B}{(q+1)} z d z=0,
$$

Eq. (19) to the equation

$$
G d G-\frac{A}{(q+1)} G d z+\frac{B}{(q+1)} z d z=0,
$$

Eq. (18) to the equation

$$
G d G+\frac{P}{(q-1)} G d z+\frac{Q}{(q-1)} z d z=0,
$$

and Eq. (20) to the equation

$$
G d G-\frac{P}{(q-1)} G d z+\frac{Q}{(q-1)} z d z=0 .
$$

Suppose,

$$
P^{2}>4 Q(q-1), \quad P>0 .
$$

For parameters $A$ and $B$, the following cases are considered:

$$
\begin{aligned}
& \text { (1) } A>0, \quad B>0 \\
& \text { (2) } A<0, \quad B>0, \quad A^{2}<4 B(q+1) .
\end{aligned}
$$

For Eqs. (23) and (24) under condition (25) the disposition of solutions is shown in Fig. 6.

For Eq. (21) under condition (26), the disposition of solutions is shown in Fig. 7 and under condition (27) in Fig. 8.

For Eq. (22) under condition (26) the disposition of solutions is shown in Fig. 9 and under condition (27) in Fig. 10. 

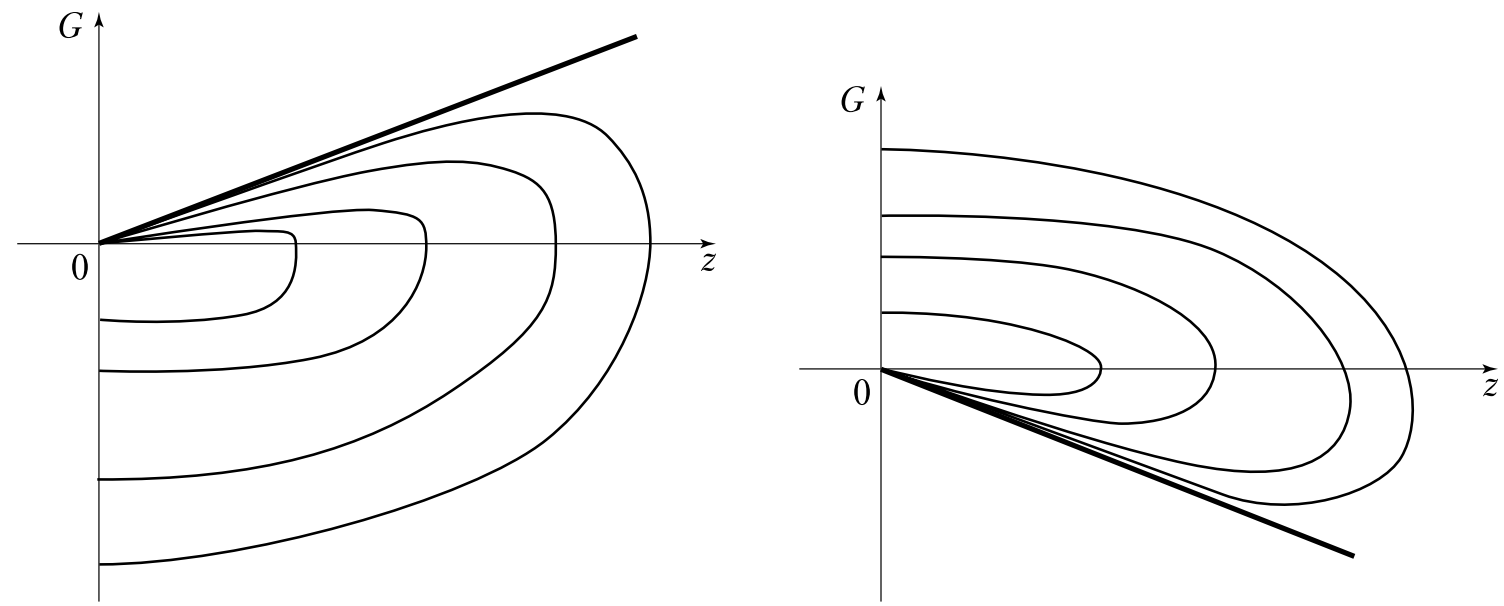

Fig. 6.
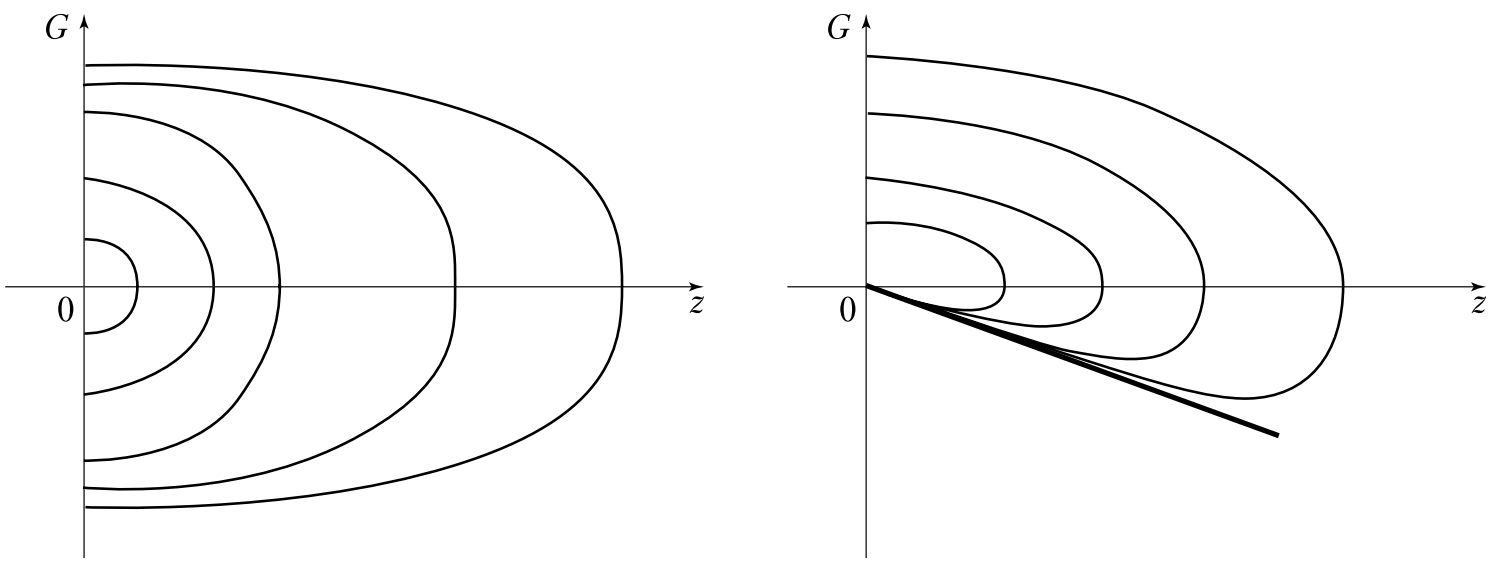

Fig. 7.

The solutions $G(z)$ of Eqs. (17)-(20) with the large initial data $G(1)=R \gg 1$ are close to the solutions of Eqs. (21)-(24). Therefore, from Figs. 610, a behavior of trajectories of system (12) can be obtained, as shown in Fig. 11.

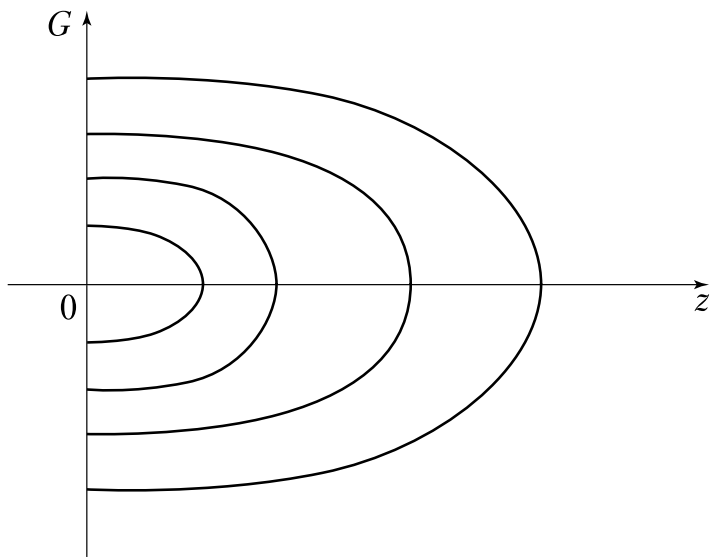

Fig. 8.
Here conditions (25)-(27) are responsible for the behavior of solutions of linear systems (17)-(20). For sufficiently large initial data, a separating solution can be obtained in the bands $-2<$ $x<-1$ and $-1<x<0$ and in the rest of the plane there are trajectories, which make a turn and are clutched by separating solutions in bands.

For trajectories' behavior considered above the following assertions can be proved.

Consider a certain fixed number $\delta>0$. Take sufficiently large number $R>0$, and introduce the following denotations

$$
\lambda=-\frac{A}{2(q+1)}, \quad \omega=\frac{\sqrt{4 B(q+1)-A^{2}}}{2(q+1)} .
$$

Lemma 1. Let conditions (27) be satisfied. Then for the solution of system (12) with the initial data $x(0)=r, u(0)=R$ there exists a number $T>0$ such that 

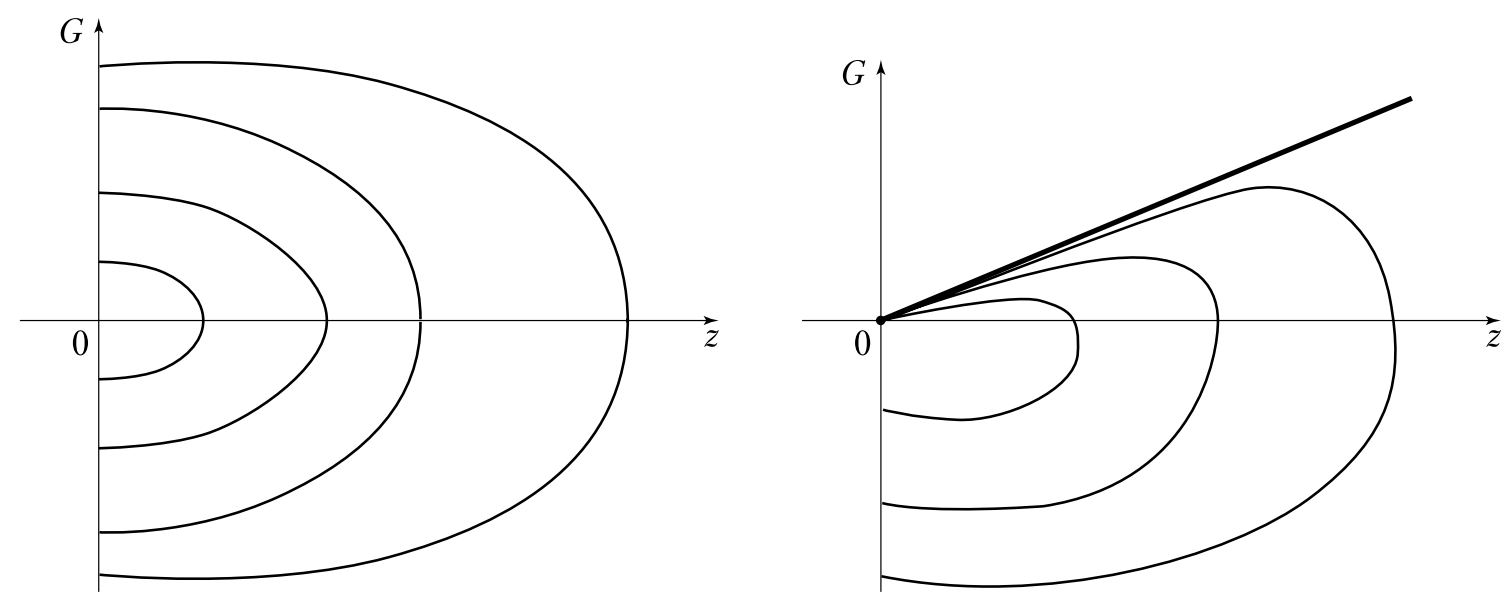

Fig. 9.

$$
\begin{gathered}
x(T)=r, \quad u(T)<0, \quad x(t)>r, \quad \forall t \in(0, T), \\
R \exp \left(\frac{\lambda \pi}{\omega}-\delta\right)<|u(T)|<R \exp \left(\frac{\lambda \pi}{\omega}+\delta\right) .
\end{gathered}
$$

The estimation of negative $F(Z)$ on $[1, Z]$ is similar to that of positive $F(Z)$ on this interval.

Take a certain number $c<-1$.

Lemma 2. Let conditions (27) be satisfied. Then for the solution of system (12) with the initial data $x(0)=r, u(0)=-R$, there exists a number $T>0$ such that

$$
\begin{gathered}
x(T)=r, \quad u(T)>0, \quad x(t)<r, \quad \forall t \in(0, T), \\
R \exp \left(\frac{\lambda \pi}{\omega}-\delta\right)<u(T)<R \exp \left(\frac{\lambda \pi}{\omega}+\delta\right) .
\end{gathered}
$$

Lemma 3. Let conditions (26) be satisfied. Then for the solution of system (12) with the initial data $x(0)=0, u(0)=R$, there exists a number $T>0$ such that

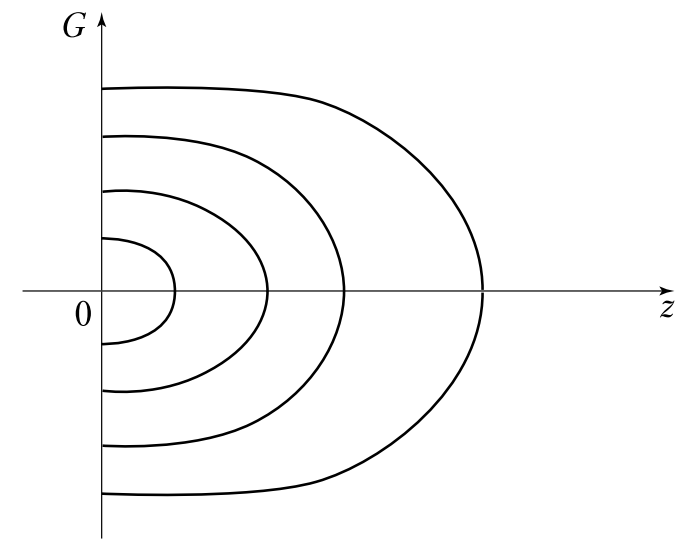

Fig. 10 .

$$
\begin{gathered}
x(T)=0, \quad u(T)<0, \quad x(t)>0, \quad \forall t \in(0, T), \\
-\delta R<u(T)<0 .
\end{gathered}
$$

Lemma 4. Let conditions (26) be satisfied. For the solution of system (12) with the initial data $x(0)=r, u(0)=-R$, there exists a number $T>0$ such that

$$
\begin{gathered}
x(T)=r, \quad u(T)>0, \quad x(t)<r, \quad \forall t \in(0, T), \\
0<u(T)<\delta R .
\end{gathered}
$$

Lemma 5. Let conditions (25) be satisfied. Then for the solution of system (12) with the initial data $x(0)=0, u(0)=-R$, there exists a number $T>0$ such that

$$
\begin{aligned}
& x(T)=0, \quad 0<u(T)<\delta R, \\
& x(t) \in(-1,0), \quad \forall t \in(0, T) .
\end{aligned}
$$

A similar assertion occurs for the case $x<-1$.

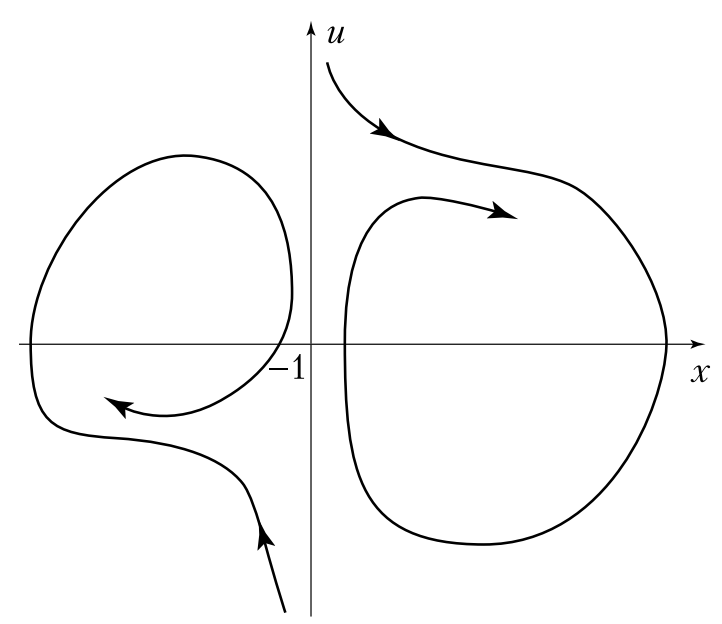

Fig. 11. 
Lemma 6. Let condition (25) be satisfied. Then for the solution of system (12) with the initial data $x(0)=r, u(0)=R$, there exists a number $T>0$ such that

$$
\begin{aligned}
& x(T)=r, \quad-\delta R<u(T)<0, \\
& x(t) \in(r,-1), \quad \forall t \in(0, T) .
\end{aligned}
$$

The proofs of lemmas are based on the qualitative behavior of trajectories, which was considered above (for details, see [Leonov, 2010a; Leonov \& Kuznetsova, 2010]).

Asymptotic analysis, of the existence of separating solutions in the bands $-2<x<-1$ and $-1<x<0$, is an extension of the approach to the study of critical saddle point at infinity under the mapping on the Poincaré sphere [Artes et al., 2008]. The ideas used were proposed in [Leonov, 2009a, 2010a] and further developed in [Leonov \& Kuznetsov, 2010].

\subsection{Global analysis: Boundedness of solutions, existence of one and two limit cycles}

For quadratic system (11) there occur the relations

$$
\begin{gathered}
A=2 c-b-1, \quad B=-a+b-c, \\
P=1+2 c, \quad Q=-c, \quad q=-c .
\end{gathered}
$$

It follows that for any $c>0$, the relation $(25)$ is satisfied.

Using the asymptotic integration method, for the above values $A$ and $B$, the following results can be obtained.

Formulate first the boundedness conditions of trajectories of system (11) [Leonov, 2010b].

Theorem 1. Let $c \neq 0, c \neq-1, c \neq b-a$. Then for the boundedness on $(0,+\infty)$ of any solution of system (11) with initial data from $\Gamma_{x>-1}$, it is necessary that $c \in(0,1)$.

Theorem 2. Let $c \neq 0, c \neq-1, c \neq b-a$. Then for the boundedness on $(0,+\infty)$ of all solutions of system (11) with initial data from $\Gamma_{x>-1}$, it is necessary and sufficient that

$$
c \in(0,1)
$$

and

$$
\begin{aligned}
& \text { either } 2 c>b+1, \quad c<b-a, \\
& \text { either } 2 c \leq b+1, \quad 4 a(c-1)>(b-1)^{2} .
\end{aligned}
$$

Here, conditions (29) of Theorem 2 correspond to conditions (26), and conditions (30) to conditions (27). Therefore, the behavior of trajectories is the same as shown in Fig. 11.

By the conditions of the boundedness of solutions, given in Theorems 1 and 2, the existence criteria of one and two large limit cycles can be formulated.

Theorem 3. Suppose that conditions (28), and (29) or (30) are satisfied and the function $g(x)$ has one zero $x=0$ on the interval $(-1,+\infty)$, which corresponds to unstable equilibrium of system (12). Then system (11) has a limit cycle in the half-plane $\Gamma_{x>-1}$.

Theorem 4. Suppose that conditions (28) and (30) are satisfied and the function $g(x)$ has only two zeros $x=0$ and $x=x_{1} \in(-\infty,-1)$, which correspond to unstable equilibria $x=u=0$ and $x=x_{1}$, $u=0$ of system (12). Then system (11) has two limit cycles. One of them is situated in the halfplane $\Gamma_{x>-1}$, another in the half-plane $\Gamma_{x<-1}$.

Note that in the case when conditions (29) are satisfied, system (12) has no unstable equilibrium in the half-plane $\Gamma_{x<-1}$.

Consider the constructive conditions under which the function $g(x)$ has only two zeros: $x=0$ and $x=x_{1} \in(-\infty,-1)$.

Proposition 5. Let the conditions of Theorem 3 or Theorem 4 be valid. In order that $g(x)$ has only two zeros, $x=0$ and $x_{1} \in(-\infty,-1)$, it is necessary and sufficient that the inequality

$$
\alpha<\lambda(a, b, c, \beta)
$$

is satisfied. Here $\lambda$ is a minimal root of the equation

$$
\begin{aligned}
\triangle(\lambda)= & -4 c \lambda^{3}+\left(-\beta^{2}+(2 b+6 c) \beta+27 c^{2}\right. \\
& \left.+(12 a-18 b) c-b^{2}\right) \lambda^{2} \\
& -2\left(-\beta^{3}+(-3 c-a+4 b) \beta^{2}\right. \\
& +\left(-5 b^{2}+9 b c+2 b a-3 a c\right) \beta-a b^{2} \\
& \left.+2 b^{3}-9 a b c+6 a^{2} c\right) \lambda \\
& +\left(-\beta^{4}+(2 b-4 c+2 a) \beta^{3}+\left(12 a c-b^{2}\right.\right. \\
& \left.-a^{2}-4 a b\right) \beta^{2}+\left(2 a^{2} b-12 a^{2} c+2 a b^{2}\right) \beta \\
& \left.-a^{2} b^{2}+4 a^{3} c\right) .
\end{aligned}
$$


Proof. It is obvious that in the case when conditions (25), and (26) or (27) are valid the relations

$$
\begin{aligned}
\operatorname{sign}\left(\lim _{x \rightarrow-1+0} g(x)\right) & =\operatorname{sign} Q=-1, \\
\operatorname{sign}\left(\lim _{x \rightarrow+\infty} g(x)\right) & =\operatorname{sign} B=+1, \\
\operatorname{sign}\left(\lim _{x \rightarrow-\infty} g(x)\right) & =-\operatorname{sign} B=-1, \\
\operatorname{sign}\left(\lim _{x \rightarrow-1-0} g(x)\right) & =-\operatorname{sign} Q=+1
\end{aligned}
$$

are satisfied. This implies the existence of two zeros $x=0$ and $x=x_{1}$. For these zeros to be unique, it is necessary and sufficient that the polynomial

$$
\begin{aligned}
-\frac{\Phi(x)}{x}= & (x+1)^{2}(a x+\alpha) \\
& -(x+1)(b x+\beta)+c x^{3}
\end{aligned}
$$

has only one real root.

Rewrite polynomial (33) as

$$
\begin{aligned}
& \tilde{a} x^{3}+\tilde{b} x^{2}+\tilde{c} x+\tilde{d}, \\
& \tilde{a}=a-b+c, \quad \tilde{b}=\alpha+2 a-b-\beta, \\
& \tilde{c}=2 \alpha+a-\beta, \quad \tilde{d}=\alpha .
\end{aligned}
$$

From Cardano's formulas it follows that the unicity condition of real root of this polynomial is the following inequality

$$
\triangle=\tilde{4} c^{3} \tilde{a}-\tilde{c}^{2} \tilde{b}^{2}-18 \tilde{a} \tilde{b} \tilde{c} \tilde{d}+27 \tilde{a}^{2} \tilde{d}^{2}+4 \tilde{b}^{3} \tilde{d}>0 .
$$

If one represents the left-hand side of this inequality in the form of polynomial of degree 3 in $\alpha$, then polynomial (32) can be obtained. Obviously, in the case when (31) is valid, where $\lambda$ is a minimal root of equation $\triangle(\lambda)=0$, the inequality $\triangle(\alpha)>0$ is satisfied. This implies the assertion of Proposition 5.

Proposition 6. If $\beta>0$, then the equilibrium $x=$ $y=0$ is Lyapunov unstable.

Proposition 7. Let the conditions of Proposition 5 be valid. For the equilibrium $x=x_{1}, y=0$ to be Lyapunov unstable, it is necessary and sufficient that $p<-1$, where $p$ is a minimal root of the equation

$$
(2 c-b-1) \tilde{P}^{2}-(2+b+\beta) \tilde{P}-\beta=0
$$

and

$$
\begin{aligned}
\alpha< & \frac{1}{(p+1)^{2}}\left(-a(p+1)^{2} p\right. \\
& \left.+(b p+\beta)(p+1) p-c p^{3}\right) .
\end{aligned}
$$

Note that from the conditions of Proposition 5, it follows that $\alpha<0$.

By Theorem 3 or 4 , together with Proposition 5 , in the space of parameters the sets $\Omega_{1}$ and $\Omega_{2}$ can be selected with one and two limit cycles, respectively. It is obvious that these sets have infinite Lebesgue measure and the sets $\Omega_{1}$ and $\Omega_{2}$, well described here, are not small.

Below, an extension of Lienard's theorem will be given. For this purpose, in system (12) on the functions $f(x)$ and $g(x)$, the following conditions will be imposed.

Suppose, the functions $f$ and $g$ are differentiable on $(-1,+\infty)$ and for certain numbers $\nu_{1} \in$ $(-1,0)$ and $\nu_{2} \in(0,+\infty)$ the following relations

$$
\begin{gathered}
g(x)<0, \quad \forall x \in(-1,0), \\
g(x)>0, \quad \forall x \in(0,+\infty), \\
\lim _{x \rightarrow-1} \int_{0}^{x} g(z) d z=\lim _{x \rightarrow+\infty} \int_{0}^{x} g(z) d z=+\infty, \\
f(x)>0, \quad \forall x \in\left(-1, \nu_{1}\right) \cup\left(\nu_{2},+\infty\right), \\
\int_{\nu_{1}}^{\nu_{2}} f(z) d z \geq 0
\end{gathered}
$$

are satisfied.

Theorem 5 [Leonov, 2006, 2008a, 2010a]. Let conditions (35) be satisfied and the equilibrium $x=u=$ 0 be unstable. Then system (12) has a limit cycle.

This theorem can be proven by asymptotic integration method or by Lyapunov direct method.

It is also obvious that Theorem 3 is a corollary of Theorem 5 .

\subsection{Visualization of limit cycles in quadratic system}

Let us apply Theorem 3 to solving the HilbertKolmogorov problem and give some numerical examples. Suppose that $a=-1, b=0, c=3 / 4$, $\beta=1$. Then (31) and (32) in system (11) yields

$$
\triangle=-3 \alpha^{3}+\frac{155}{16 \alpha^{2}}-9 \alpha-28, \quad \lambda \approx-1.156 .
$$

In this case for $\alpha<-1.2$, conditions (31), (28), and (29) are satisfied, and one can visualize a limit 

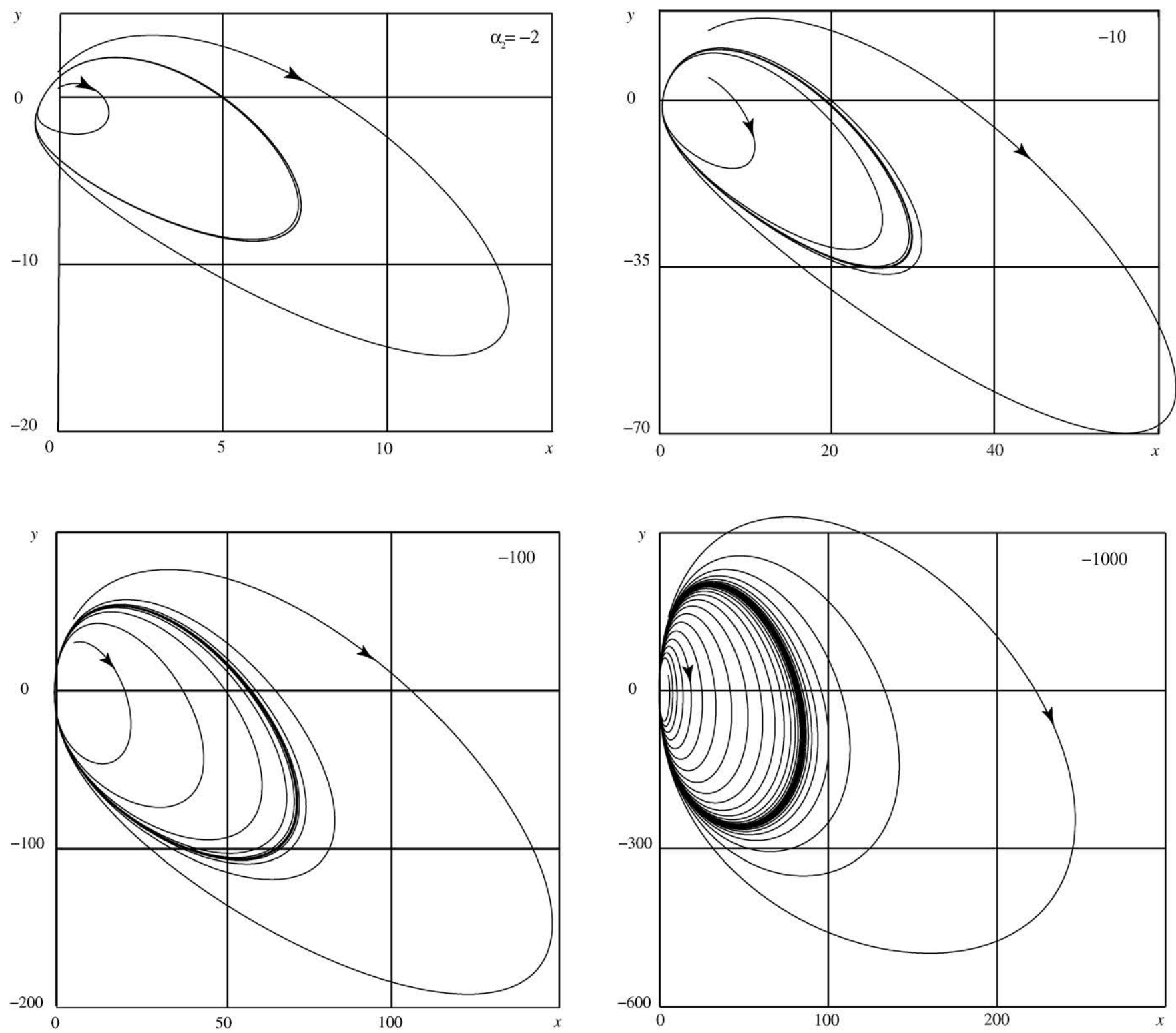

Fig. 12. Localization of large limit cycles.

cycle. For $\alpha=-2,-10,-100,-1000$, the limit cycles are shown in Fig. 12.

Naturally, any student could obtain these results, if Kolmogorov might give her/him a task with such parameters. In this case, the limit cycles "are well seen". They were obtained by virtue of the following goal-oriented operations. For various types of Lienard equations, describing the dynamics of mechanical, electromechanical, and electronic systems, the existence conditions of globally stable limit cycles were well known [Cesari, 1959; Lefschetz, 1957; Migulin et al., 1978]. Therefore, when it became clear that quadratic system can be reduced to special Lienard equation, the following natural step consists of an attempt to extend these results (see [Leonov, 2006, 2008b]) to the previous classical investigations [Cesari, 1959; Lefschetz, 1957; Bogolyubov \& Mitropolskii, 1961; Migulin et al., 1978]. Such an extension allows one to obtain the existence of limit cycle conditions, which select a set of infinite Lebesgue measure in parameters space of quadratic system (11). Remark that this set is not "small".

Theorem 4 on a global behavior of trajectories on phase plane is well combined with the local 
analysis of "small" limit cycles, which will be presented below.

\subsection{Local analysis: Computation of Lyapunov values and small limit cycles}

\subsubsection{Lyapunov values definition}

The computation of Lyapunov value was proposed in the classical investigations of Poincaré [Poincaré, 1885] and Lyapunov [Lyapunov, 1892], devoted to the analysis of stability of degenerated (or weak) focus equilibrium. A sign of Lyapunov value defines winding/unwinding of solutions of systems in small neighborhoods of equilibrium and stability/instability of equilibrium.

In the middle of last century, Bautin proposed first the effective method, based on the computation and sequential perturbation of Lyapunov values, for the construction of polynomial systems with nested limit cycles, and gave an example of quadratic system with three nested limit cycles [Bautin, 1949, 1952]. After that, the analysis of Lyapunov values became one of the central problems in considering limit cycles in the neighborhood of equilibrium of two-dimensional dynamical systems (see, e.g. [Marsden \& McCracken, 1976; Lloyd, 1988; Yu, 1998; Giné \& Santallusia, 2004; Dumortier et al., 2006; Christopher \& Li, 2007; Yu \& Chen, 2008; Li et al., 2008; Borodzik \& Żołądek, 2008; Yu \& Corless, 2009; Li et al., 2012; Giné, 2012; Shafer, 2009] and others).

Probably because of different translations of Bautin's works from Russian and the large number of scientists who simultaneously started to develop his technique by various methods there are several terms (Liapunov or Lyapunov quantities or coefficients, Poincaré or Poincaré-Lyapunov constants, focus values, foci values and others), which are being used for the characterization of behavior of degenerate focus. The present authors believe that it is natural to use the term Lyapunov values or Lyapunov focus values since they are further extensions of the term eigenvalue, and the first approach to introducing Lyapunov values was based on the construction of the Lyapunov function (it is described below).

Note that although scholars began to consider the problem of symbolic computation of Lyapunov values (the expressions in terms of coefficients of the right-hand side of the considered dynamical system) in the first half of the last century, substantial progress in the study of Lyapunov values became possible only in the past decade by virtue of the use of modern software tools of symbolic computation. While general expressions for the first and second Lyapunov values (in terms of coefficient expansion of right-hand side of the considered dynamical system) were obtained in the 4050s of the last century in the works [Bautin, 1949; Serebryakova, 1959], the general expression of the third Lyapunov value was computed only in 2008 [Kuznetsov \& Leonov, 2008a; Leonov et al., 2011a] and occupies more than four pages.

Introduce Lyapunov values following [Kuznetsov \& Leonov, 2008b; Kuznetsov, 2008; Leonov et al., 2011a,a]. Consider a two-dimensional system of autonomous differential equations

$$
\begin{aligned}
& \frac{d x}{d t}=f_{10} x+f_{01} y+f(x, y), \\
& \frac{d y}{d t}=g_{10} x+g_{01} y+g(x, y),
\end{aligned}
$$

where $x, y \in \mathbb{R}$ and $f(0,0)=0, g(0,0)=0$. Suppose that the functions $f(\cdot, \cdot)$ and $g(\cdot, \cdot)$ are sufficiently smooth and their expansions begin with the terms of at least second order, namely

$$
\begin{aligned}
f(x, y) & =\sum_{k+j=2}^{n} f_{k j} x^{k} y^{j}+o\left((|x|+|y|)^{n}\right) \\
& =f_{n}(x, y)+o\left((|x|+|y|)^{n}\right), \\
g(x, y) & =\sum_{k+j=2}^{n} g_{k j} x^{k} y^{j}+o\left((|x|+|y|)^{n}\right) \\
& =g_{n}(x, y)+o\left((|x|+|y|)^{n}\right) .
\end{aligned}
$$

Let the first approximation matrix $A_{(0,0)}=$ $\left(\begin{array}{ll}f_{10} & f_{01} \\ g_{10} & g_{01}\end{array}\right)$ of the system have two purely imaginary eigenvalues. In this case, without loss of generality (i.e. there is a nonsingular linear change of variables), it can be assumed that $f_{10}=0, f_{01}=$ $-1, g_{10}=1, g_{01}=0$. Then system (36) takes the form

$$
\begin{aligned}
& \frac{d x}{d t}=-y+f(x, y), \\
& \frac{d y}{d t}=x+g(x, y) .
\end{aligned}
$$


Consider, following Poincaré method, the intersection of trajectory of system (38) with the straight line $x=0$. At time $t=0$, the trajectory $(x(t, h)$, $y(t, h))$ starts from the point $(0, h)$ ( $h$ is sufficiently small)

$$
(x(0, h), y(0, h))=(0, h) .
$$

Denote by $T(h)$ a return time of trajectory $(x(t, h)$, $y(t, h))$, which is a time between two successive intersections of the trajectory with the straight line $x=0$. Note that for sufficiently small $h$ the return time can be found and it is finite since the righthand side of system (38) and its linear part differ by $o(|x|+|y|)$ in the neighborhood of zero (and the return time for linear system is $2 \pi$ ). Then

$$
x(T(h), h)=0
$$

and $y(T(h), h)$ can be sequentially approximated by a series in terms of powers of $h$ :

$$
y(T(h), h)=h+\tilde{L}_{2} h^{2}+\tilde{L}_{3} h^{3}+\cdots .
$$

Here the first nonzero coefficient $\tilde{L}_{m}$ is called Lyapunov value. It indicates an influence of nonlinear terms $f(x, y)$ and $g(x, y)$ on the behavior of trajectories of system (38) in a small neighborhood of stationary point. Lyapunov value defines a stability or instability of stationary point and describes a winding or unwinding of trajectory (Fig. 13). It can be shown (see, e.g. [Lyapunov, 1892]) that the first nonzero coefficient has a necessarily odd number $m=(2 k+1)$. The value $\tilde{L}_{2 k+1}$ is called $k$ th Lyapunov value

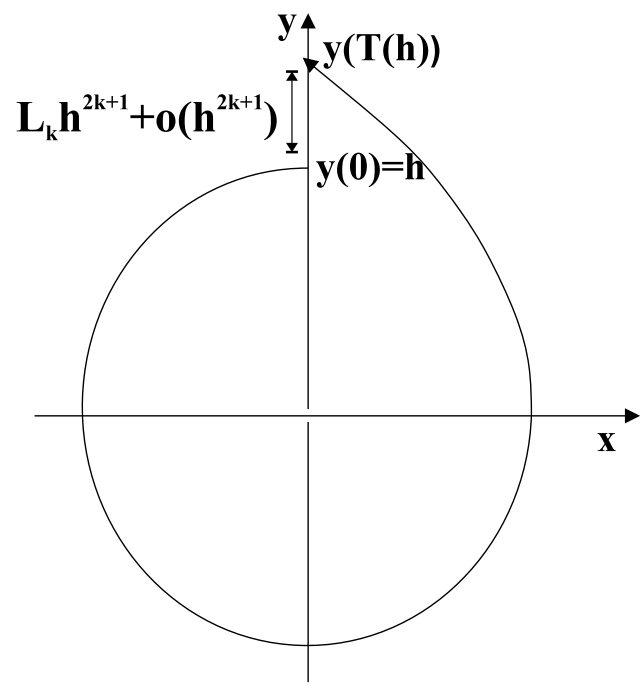

Fig. 13. Focus stationary point and Lyapunov value.

$$
L_{k}=\tilde{L}_{2 k+1}
$$

and the equilibrium is called a weak focus of order $k$.

In the case when the complex eigenvalues of the first approximation matrix of system have a real part, the notion of Lyapunov value is defined similarly. In this case, a notion of zero Lyapunov value $L_{0}=\tilde{L}_{1}$ is introduced such that

$$
y(T(h), h)=\left(1+\tilde{L}_{1}\right) h+o(h) .
$$

Note that $\tilde{L}_{1}$ describes an exponential increase of system solutions, caused by the real parts of eigenvalues (similarly to Lyapunov exponents or characteristic exponents [Leonov \& Kuznetsov, 2007b]).

In addition, following Lyapunov [1892], in the case when a linear system has two purely imaginary roots and the rest of roots is negative, a similar procedure for the study of stability can be used for systems of larger dimension.

At present, there are various methods for computing Lyapunov values and for the computer realizations of these methods. The methods considered differ in the complexity of algorithms, the compactness of the obtained symbolic expressions, and a space in which the computations are performed. Two main ideas, on which the methods are based, are the construction of approximations of system solutions for the analysis of Poincaré map and the construction of a local Lyapunov function.

For simplicity of computations, various changes of variables and the reduction to normal forms are often applied for the original system first. This permits one to simplify computations and to obtain more compact expressions for Lyapunov values of the transformed system (see, e.g. [Serebryakova, 1959; Gasull et al., 1997; Yu, 1998]). However, the analysis of original system in non-original space becomes less demonstrative.

The modern computers and symbolic computations allow one to effectively use these methods and to find Lyapunov values in the form of symbolic expressions, depending on the expansion coefficients of the right-hand sides of system (38) (see, e.g. [Lloyd, 1988; Gasull et al., 1997; Roussarie, 1998; Yu, 1998; Chavarriga \& Grau, 2003; Lynch, 2010; Giné, 2007; Christopher \& Li, 2007; Leonov \& Kuznetsov, 2007a; Yu \& Chen, 2008; Kuznetsov \& Leonov, 2008b; Kuznetsov, 2008]).

Here, two constructive methods are considered for the computation of Lyapunov values, which 
permit one to compute Lyapunov values in the initial "physical" space (this is often important for the study of applied problems). The advantages of these methods are an ideological simplicity and visualization.

\subsubsection{Direct method for computation of Lya-} punov values in Euclidean coordinates and in the time domain

Direct method for the computation of Lyapunov values was suggested in [Kuznetsov \& Leonov, 2008b; Leonov et al., 2011a]. It is based on the construction of solution approximations (as a finite sum in powers of initial datum) in the original Euclidean coordinates and in the time domain.

This approach can also be applied to the problem of distinguishing the isochronous center (see, e.g. [Gasull et al., 1997; Sabatini \& Chavarriga, 1999; Chavarriga \& Grau, 2003; Giné, 2007; Pearson \& Lloyd, 2009; Feng \& Yirong, 2012]) since it permits one to find an approximation of return time of trajectory as a function of the initial data.

In the case when smoothness condition (37) is satisfied, the functions $x(t, h)$ and $y(t, h)$ can be presented as

$$
\begin{aligned}
x(t, h) & =x_{h^{n}}(t, h)+o\left(h^{n}\right) \\
& =\sum_{k=1}^{n} \tilde{x}_{h^{k}}(t) h^{k}+o\left(h^{n}\right), \\
y(t, h) & =y_{h^{n}}(t, h)+o\left(h^{n}\right) \\
& =\sum_{k=1}^{n} \tilde{y}_{h^{k}}(t) h^{k}+o\left(h^{n}\right) .
\end{aligned}
$$

Here $x_{h^{1}}(t, h)=\tilde{x}_{h^{1}}(t) h=-h \sin (t), y_{h^{1}}(t, h)=$ $\tilde{y}_{h^{1}}(t) h=h \cos (t)$ and $\tilde{x}_{h^{k}}(t), \tilde{y}_{h^{k}}(t)$ can be found sequentially by virtue of the following.

Lemma 7. Consider the following system

$$
\begin{gathered}
\frac{d \tilde{x}_{h^{k}}(t)}{d t}=-\tilde{y}_{h^{k}}(t)+u_{h^{k}}^{f}(t), \\
\frac{d \tilde{y}_{h^{k}}(t)}{d t}=\tilde{x}_{h^{k}}(t)+u_{h^{k}}^{g}(t) .
\end{gathered}
$$

For the solutions of system (43) with the initial data

$$
\tilde{x}_{h^{k}}(0)=0, \quad \tilde{y}_{h^{k}}(0)=0
$$

the equations

$$
\begin{aligned}
\tilde{x}_{h^{k}}(t)= & u_{h^{k}}^{g}(0) \cos (t) \\
& +\cos (t) \int_{0}^{t} \cos (\tau)\left(\left(u_{h^{k}}^{g}(\tau)\right)^{\prime}+u_{h^{k}}^{f}(\tau)\right) \mathrm{d} \tau \\
& +\sin (t) \int_{0}^{t} \sin (\tau)\left(\left(u_{h^{k}}^{g}(\tau)\right)^{\prime}+u_{h^{k}}^{f}(\tau)\right) \mathrm{d} \tau \\
& -u_{h^{k}}^{g}(t), \\
\tilde{y}_{h^{k}}(t)= & u_{h^{k}}^{g}(0) \sin (t) \\
& +\sin (t) \int_{0}^{t} \cos (\tau)\left(\left(u_{h^{k}}^{g}(\tau)\right)^{\prime}+u_{h^{k}}^{f}(\tau)\right) \mathrm{d} \tau \\
& -\cos (t) \int_{0}^{t} \sin (\tau)\left(\left(u_{h^{k}}^{g}(\tau)\right)^{\prime}+u_{h^{k}}^{f}(\tau)\right) \mathrm{d} \tau
\end{aligned}
$$

are valid.

Here $u_{h^{k}}^{f}(t), u_{h^{k}}^{g}(t)$ can be found by the substitution of $x(t, h)=x_{h^{k-1}}(t, h)+o\left(h^{k-1}\right), y(t, h)=$ $y_{h^{k-1}}(t, h)+o\left(h^{k-1}\right)$ into $f$ and $g$

$$
\begin{aligned}
& f\left(x_{h^{k-1}}(t, h)+o\left(h^{k-1}\right), y_{h^{k-1}}(t, h)+o\left(h^{k-1}\right)\right) \\
& =u_{h^{k}}^{f}(t) h^{k}+o\left(h^{k}\right), \\
& g\left(x_{h^{k-1}}(t, h)+o\left(h^{k-1}\right), y_{h^{k-1}}(t, h)+o\left(h^{k-1}\right)\right) \\
& \quad=u_{h^{k}}^{g}(t) h^{k}+o\left(h^{k}\right) .
\end{aligned}
$$

Consider return time $T(h)$ for the initial datum $h \in(0, H]$, and define that $T(0)=2 \pi$. It can be proved that $T(h)$ is $n$ times differentiable function. Thus

$$
T(h)=2 \pi+\Delta T=2 \pi+\sum_{k=1}^{n} \tilde{T}_{k} h^{k}+o\left(h^{n}\right),
$$

where $\tilde{T}_{k}=\frac{1}{k !} \frac{d^{k} T(h)}{d h^{k}}$ (the so-called period constants [Giné, 2007]).

Substitute relation (46) for $t=T(h)$ on the right-hand side of the first equation of (42), and denote the coefficients of $h^{k}$ by $\tilde{x}_{k}$. Then the series $x(T(h), h)$ can be obtained in terms of powers of $h$ :

$$
x(T(h), h)=\sum_{k=1}^{n} \tilde{x}_{k} h^{k}+o\left(h^{n}\right) .
$$

In order to express the coefficients $\tilde{x}_{k}$ by the coefficients $\tilde{T}_{k}$, it is assumed that in the first equation 
of $(42) t=2 \pi+\tau$. Then

$$
x(2 \pi+\tau, h)=\sum_{k=1}^{n} \tilde{x}_{h^{k}}(2 \pi+\tau) h^{k}+o\left(h^{n}\right) .
$$

Here by smoothness condition (37)

$$
\begin{aligned}
\tilde{x}_{h^{k}}(2 \pi+\tau)= & \tilde{x}_{h^{k}}(2 \pi)+\sum_{m=1}^{n} \tilde{x}_{h^{k}}^{(m)}(2 \pi) \frac{\tau^{m}}{m !} \\
& +o\left(\tau^{n}\right), \quad k=1, \ldots, n .
\end{aligned}
$$

Substitution of this representation in (48) for $\tau=$ $\Delta T(h)$ and grouping together of coefficients with the same power of $h$ give

$$
\begin{aligned}
h: 0= & \tilde{x}_{1}=\tilde{x}_{h^{1}}(2 \pi), \\
h^{2}: 0= & \tilde{x}_{2}=\tilde{x}_{h^{2}}(2 \pi)+\tilde{x}_{h^{1}}^{\prime}(2 \pi) \tilde{T}_{1}, \\
h^{3}: 0= & \tilde{x}_{3}=\tilde{x}_{h^{3}}(2 \pi)+\tilde{x}_{h^{1}}^{\prime}(2 \pi) \tilde{T}_{2}+\frac{1}{2} \tilde{x}_{h^{2}}^{\prime}(2 \pi) \tilde{T}_{1} \\
& \quad+\frac{1}{2} \tilde{x}_{h^{1}}^{\prime \prime}(2 \pi) \tilde{T}_{1}^{2} \\
& \vdots \\
h^{n}: 0= & \tilde{x}_{n}=\tilde{x}_{h^{n}}(2 \pi)+\tilde{x}_{h^{1}}^{\prime}(2 \pi) \tilde{T}_{n-1}+\cdots
\end{aligned}
$$

(here' denotes a derivative with respect to time $t$ ). Hence, it is possible to determine sequentially coefficients $\tilde{T}_{k=1, \ldots, n-1}$ via the coefficients $f_{i j}$ and $g_{i j}$ since in the expression for $\tilde{x}_{k}$ there is only one addend $\tilde{x}_{h^{1}}^{\prime}(2 \pi) \tilde{T}_{k-1}=-\tilde{T}_{k-1}$, which includes $\tilde{T}_{k-1}$, and the rest of the expression depends on $\tilde{T}_{1 \leq m<k-1}$.

By a similar procedure, the coefficients $\tilde{y}_{k}$ in the expansion

$$
y(T(h), h)=\sum_{k=1}^{n} \tilde{y}_{k} h^{k}+o\left(h^{n}\right)
$$

can be obtained.

Substituting the following representation

$$
\begin{aligned}
\tilde{y}_{h^{k}}(2 \pi & +\Delta T(h)) \\
= & \tilde{y}_{h^{k}}(2 \pi)+\sum_{m=1}^{n} \tilde{y}_{h^{k}}^{(m)}(2 \pi) \frac{\Delta T(h)^{m}}{m !} \\
& +o\left((\Delta T(h))^{n}\right), \quad k=1, \ldots, n
\end{aligned}
$$

into the expression

$$
\begin{aligned}
y(2 \pi & +\Delta T(h), h) \\
& =\sum_{k=1}^{n} \tilde{y}_{h^{k}}(2 \pi+\Delta T(h)) h^{k}+o\left(h^{n}\right),
\end{aligned}
$$

gives

$$
y(T(h), h)=\sum_{k=1}^{n} \tilde{y}_{k} h^{k}+o\left(h^{n}\right) .
$$

By equating the coefficients of the same power of $h$

$$
\begin{aligned}
h: \tilde{y}_{1}= & \tilde{y}_{h^{1}}(2 \pi), \\
h^{2}: \tilde{y}_{2}= & \tilde{y}_{h^{2}}(2 \pi)+\tilde{y}_{h^{1}}^{\prime}(2 \pi) \tilde{T}_{1}, \\
h^{3}: \tilde{y}_{3}= & \tilde{y}_{h^{3}}(2 \pi)+\tilde{y}_{h^{1}}^{\prime}(2 \pi) \tilde{T}_{2}+\frac{1}{2} \tilde{y}_{h^{2}}^{\prime}(2 \pi) \tilde{T}_{1} \\
& \quad+\frac{1}{2} \tilde{y}_{h^{1}}^{\prime \prime}(2 \pi) \tilde{T}_{1}^{2}, \\
& \vdots \\
h^{n}: \tilde{y}_{n}= & \tilde{y}_{h^{n}}(2 \pi)+\tilde{y}_{h^{1}}^{\prime}(2 \pi) \tilde{T}_{n-1}+\cdots,
\end{aligned}
$$

we can sequentially define $\tilde{y}_{i=1, \ldots, n}$.

Here the expressions $\tilde{y}_{h^{1}}(2 \pi)=1$ and $\tilde{T}_{k=1, \ldots, n-1}$, and the functions $\tilde{y}_{h^{k=1, \ldots, n}}(t)$ are defined above.

Note that for $n=2 m+1$, if $\tilde{y}_{k}=0$ for $k=2, \ldots, 2 m$, then $\tilde{y}_{2 m+1} \neq 0$ is $m$ th Lyapunov value:

$$
L_{m}=\tilde{y}_{2 m+1} .
$$

The algorithm considered is constructive and can easily be realized in a symbolic computation package. The realization of this method in MatLab can be found in [Kuznetsov, 2008]. Note also that this approach can easily be used to the general case of the linear part of system (38).

Example 6. Consider the Duffing equation represented as the system

$$
\dot{x}=-y, \quad \dot{y}=x+x^{3} .
$$

It is well known that all trajectories of this system are periodic (i.e. $L_{i}=0$ ). Analyze the period of periodic trajectories of this system. For $x_{0}=0$, $y_{0}=h_{y}$

$$
y(t)^{2}+x(t)^{2}+\frac{1}{2} x(t)^{4}=h_{y}^{2} .
$$

For the return time $T\left(h_{y}\right)$, from the relation $d t / d y=\left(x+x^{3}\right)^{-1}$ it follows that

$$
\begin{aligned}
& T\left(h_{y}\right) \\
& =4 \int_{0}^{h_{y}} \frac{\mathrm{d} y}{\sqrt{-1+\sqrt{1+2 h_{y}^{2}-2 y^{2}}} \sqrt{1+2 h_{y}^{2}-2 y^{2}}}
\end{aligned}
$$




$$
\begin{aligned}
& =\int_{0}^{\pi / 2} \frac{-h_{y} \sin (z) \mathrm{d} z}{\sqrt{-1+\sqrt{1+2 h_{y}^{2} \sin ^{2} z}} \sqrt{1+2 h_{y}^{2} \sin ^{2} z}} \\
& =2 \pi-\frac{3 \pi}{4} h_{y}^{2}+\frac{105 \pi}{128} h_{y}^{4}-\frac{1155 \pi}{1024} h_{y}^{6}+o\left(h_{y}^{6}\right) .
\end{aligned}
$$

The same result can be obtained by the considered above method considered. Below, we represent the approximations of solution, obtained by the above described algorithm:

$$
\begin{aligned}
& \tilde{x}_{h^{1}}(t)=-\sin (t), \quad \tilde{y}_{h^{1}}(t)=\cos (t) \\
& \tilde{x}_{h^{2}}(t)=\tilde{y}_{h^{2}}(t)=0 ; \\
& \tilde{x}_{h^{3}}(t)=\frac{1}{8} \cos (t)^{2} \sin (t)-\frac{3}{8} t \cos (t)+\frac{1}{4} \sin (t), \\
& \tilde{y}_{h^{3}}(t)=-\frac{3}{8} t \sin (t)+\frac{3}{8} \cos (t)-\frac{3}{8} \cos (t)^{3} .
\end{aligned}
$$

Here the Lyapunov values are equal to zero by virtue of (52) and a periodic solution is approximated by a series with nonperiodic coefficients.

\subsubsection{Poincaré method based on Lyapunov function construction}

Another method for computation of Lyapunov values was suggested by Poincaré [1885] and was then developed by Lyapunov [1892]. The method consists in sequentially obtaining time-independent integrals for the approximations of system. Since the expression

$$
V_{2}(x, y)=\frac{\left(x^{2}+y^{2}\right)}{2}
$$

is an integral of the first approximation of system (38) and the system is sufficiently smooth, in a certain small neighborhood of zero point, one can sequentially construct the Lyapunov function of the form

$$
V(x, y)=\frac{x^{2}+y^{2}}{2}+V_{3}(x, y)+\cdots+V_{k}(x, y) .
$$

Here $V_{k}(x, y)=\sum_{i+j=k} V_{i, j} x^{i} y^{j}$ are homogeneous polynomials with the unknown coefficients $\left\{V_{i, j}\right\}_{i+j=k, i, j \geq 0}$ and $k \leq n+1$. For the derivative $V(x, y)$ in virtue of system (38) with provision for representation (37), it can be obtained that

$$
\begin{aligned}
\dot{V}(x, y)= & \frac{\partial V(x, y)}{\partial x}\left(-y+f_{n}(x, y)\right) \\
& +\frac{\partial V(x, y)}{\partial y}\left(x+g_{n}(x, y)\right) \\
& +o\left((|x|+|y|)^{n+1}\right) .
\end{aligned}
$$

Denoting in the obtained expression, the homogeneous terms of order $k$ via $W_{k}(x, y)$ and taking into account that as per the relation $\dot{V}_{2}(x, y)=$ $x f(x, y)+y g(x, y)=o\left((|x|+|y|)^{2}\right)$, the relation $\dot{V}(x, y)=o\left((|x|+|y|)^{2}\right)$ is valid, it can be found

$$
\begin{aligned}
\dot{V}(x, y)= & W_{3}(x, y)+\cdots+W_{n+1}(x, y) \\
& +o\left((|x|+|y|)^{n+1}\right) .
\end{aligned}
$$

Here

$$
\begin{aligned}
W_{k}(x, y)= & \left(x \frac{\partial V_{k}(x, y)}{\partial y}-y \frac{\partial V_{k}(x, y)}{\partial x}\right) \\
& +u_{k}(x, y)
\end{aligned}
$$

where the coefficients of $u_{k}(x, y)$ depend on $\left\{V_{i j}\right\}_{i+j<k}$ and $\left\{f_{i j}, g_{i j}\right\}_{i+j<k}$.

These coefficients can be found sequentially (via the coefficients of expansions of the functions $f$ and $g$ and the coefficients $\left\{V_{i, j}\right\}_{i+j<k}$ obtained at the previous steps of the procedure) in such a way that the derivative of $V(x, y)$ by virtue of system (38)

$$
\begin{aligned}
\dot{V}(x, y)= & \frac{\partial V(x, y)}{\partial x}(-y+f(x, y)) \\
& +\frac{\partial V(x, y)}{\partial y}(x+g(x, y))
\end{aligned}
$$

takes the form

$$
\begin{aligned}
\dot{V}(x, y)= & w_{1}\left(x^{2}+y^{2}\right)^{2}+w_{2}\left(x^{2}+y^{2}\right)^{3} \\
& +\cdots+o\left((|x|+|y|)^{k+1}\right) .
\end{aligned}
$$

Here the coefficients $w_{i}$ depend only on the coefficients of the expansions of $f$ and $g$.

To define the coefficients $\left\{V_{i j}\right\}_{i+j=k}$ for odd $k=2 m+1$, it is necessary, generally speaking, to solve a nonhomogeneous linear system (obtained from the equation $\left.W_{2 m+1}(x, y)=0\right)$ of $(k+1)$ equations with respect to $(k+1)$ unknown coefficients, which always has a unique solution. For $k=3$, 


$$
\left(\begin{array}{l}
V_{30} \\
V_{21} \\
V_{12} \\
V_{03}
\end{array}\right)=-\left(\begin{array}{rrrr}
0 & 1 & 0 & 0 \\
-3 & 0 & 2 & 0 \\
0 & -2 & 0 & 3 \\
0 & 0 & -1 & 0
\end{array}\right)^{-1}\left(\begin{array}{c}
f_{20} \\
g_{20}+f_{11} \\
f_{02}+g_{11} \\
g_{02}
\end{array}\right) \text {. }
$$

To define the coefficients $\left\{V_{i j}\right\}_{i+j=k}$ for even $k=(2 m+2)$, it is necessary, generally speaking, to solve a nonhomogeneous linear system (obtained from the relation $W_{2 m+2}(x, y)-w_{m}\left(x^{2}+\right.$ $\left.\left.y^{2}\right)^{m+1}=0\right)$ of $(k+1)$ equations with respect to $(k+2)$ unknown coefficients: $(k+1)$ coefficients $\left\{V_{i j}\right\}_{i+j=k}$ and $w_{m}$. For the unknown coefficients to be defined uniquely, the equations

$$
\begin{array}{rlrl}
V_{(m+1)(m+1)} & =0, & & \text { if } m \text { odd } \\
V_{(m)(m+2)}+V_{(m+2)(m)} & =0, & \text { if } m \text { even, }
\end{array}
$$

can be added [Lynch, 2010]. Note that the relation $W_{2 m+2}(x, y)=0$ leads to a linear system, the matrix rank of which is equal to $2 m+1$ (unlike for odd $k$ ). For $k=4$

$$
\begin{aligned}
\left(\begin{array}{c}
V_{4,0} \\
V_{3,1} \\
w_{4} \\
V_{1,3} \\
V_{0,4}
\end{array}\right)= & -\left(\begin{array}{rrrrr}
0 & 1 & -1 & 0 & 0 \\
-4 & 0 & 0 & 0 & 0 \\
0 & -3 & -2 & 3 & 0 \\
0 & 0 & 0 & 0 & 4 \\
0 & 0 & -1 & -1 & 0
\end{array}\right)^{-1} \\
& \times\left(\begin{array}{c}
f_{30}+V_{21} g_{20}+3 V_{30} f_{20} \\
V_{21} g_{02}+V_{12} f_{20}+3 V_{30} f_{02}+2 V_{12} g_{11}+2 V_{21} f_{11}+3 V_{03} g_{20}+g_{21}+f_{12} \\
2 V_{21} f_{02}+3 V_{03} g_{11}+V_{12} f_{11}+g_{12}+f_{03}+2 V_{12} g_{02} \\
g_{03}+3 V_{03} g_{02}+V_{12} f_{02}
\end{array}\right) .
\end{aligned}
$$

Sequentially defining the coefficients of the form $V_{k}$ for $k=3,4, \ldots$ from (55) and (56), one can obtain a coefficient $w_{m}$, which is the first non-zero coefficient $\left(2 \pi w_{m}\right.$ is equal to the $m$ th Lyapunov value [Frommer, 1928]). This coefficient is called a Lyapunov or Poincaré-Lyapunov constant [Sabatini \& Chavarriga, 1999; Chavarriga \& Grau, 2003]. If such a constant $w_{m} \neq 0$ is obtained, then in a certain small neighborhood of zero the derivative $V(x, y)$ as per the system has constant sign (its sign coincides with the sign of $\left.w_{m}\right)$, and $V(x, y)$ is sign definite (i.e. the function constructed satisfies locally the conditions of Lyapunov theorem on stability and instability)

$$
\begin{aligned}
V(x, y)= & \frac{x^{2}+y^{2}}{2}+o\left((|x|+|y|)^{2}\right), \\
\dot{V}(x, y)= & w_{m}\left(x^{2}+y^{2}\right)^{m+1} \\
& +o\left((|x|+|y|)^{2 m+2}\right) .
\end{aligned}
$$

A known modification of the Poincaré-Lyapunov method is a transition to complex variables (see, e.g. [Schuko, 1968; Gasull et al., 1997; Li et al., 2008; Huang et al., 2008]).

\subsubsection{Lyapunov values of Lienard system}

Consider system (36), where $g_{10}$ is an arbitrary number.

Suppose that

$$
\begin{gathered}
f_{10}=0, \quad f_{01}=-1, \quad f(x, y) \equiv 0 \\
g_{01}=0, \quad g(x, y)=g_{x 1}(x) y+g_{x 0}(x)
\end{gathered}
$$

and

$$
\begin{aligned}
& g_{x 1}(x)=g_{11} x+g_{21} x^{2}+\cdots, \\
& g_{x 0}(x)=g_{20} x^{2}+g_{30} x^{3}+\cdots .
\end{aligned}
$$

Then one gets a Lienard system in general form

$$
\dot{x}=-y, \quad \dot{y}=g_{10} x+g_{x 1}(x) y+g_{x 0}(x) .
$$

Note, in order that the matrix of linear approximation of system has two purely imaginary eigenvalues, the following condition is necessary to be satisfied

$$
g_{10}>0 .
$$

Since the methods for the computation of Lyapunov values are described above for the systems with simple linear part $\left(g_{10}=1\right)$, one can transform 
system (57) to the required form by the following change of variables

$$
t \rightarrow \sqrt{\frac{1}{g_{10}}} t, \quad x \rightarrow \sqrt{\frac{1}{g_{10}}} x .
$$

This change of variables does not change $y$ [see (57)], namely

$$
y=-\frac{\sqrt{\frac{1}{g_{10}}} d x}{\sqrt{\frac{1}{g_{10}}} d t}=-\frac{d x}{d t}
$$

and, therefore, representation of $y$ (49) is just as before. It means that the expressions for Lyapunov values for the systems before and after the change of variables coincide.
For the case of Lienard system of general form under condition (58), the expressions for the Lyapunov values $L_{i=1, \ldots, 4}$ will be given below.

The first Lyapunov value is as follows

$$
L_{1}=\frac{\pi}{4\left(g_{10}\right)^{5 / 2}}\left(g_{21} g_{10}-g_{11} g_{20}\right) .
$$

If $g_{21}=\frac{g_{11} g_{20}}{g_{10}}$, then $L_{1}=0$ and it can be obtained,

$$
\begin{aligned}
L_{2}= & \frac{-\pi}{24\left(g_{10}\right)^{9 / 2}}\left(3 g_{11} g_{10} g_{40}-3 g_{41} g_{10}{ }^{2}\right. \\
& \left.+5 g_{20} g_{10} g_{31}-5 g_{30} g_{11} g_{20}\right) .
\end{aligned}
$$

If $g_{41}=\frac{3 g_{11} g_{10} g_{40}+5 g_{20} g_{10} g_{31}-5 g_{30} g_{11} g_{20}}{3 g_{10}{ }^{2}}$, then $L_{2}=0$ and it can be obtained

$$
\begin{aligned}
L_{3}= & \frac{-\pi}{576\left(g_{10}\right)^{15 / 2}}\left(63 g_{40} g_{10}{ }^{3} g_{31}-70 g_{20}{ }^{3} g_{10} g_{31}-105 g_{50} g_{10}{ }^{2} g_{11} g_{20}+105 g_{20} g_{10}{ }^{3} g_{51}-45 g_{61} g_{10}{ }^{4}\right. \\
& \left.-105 g_{30} g_{10}{ }^{2} g_{20} g_{31}-63 g_{30} g_{10}{ }^{2} g_{11} g_{40}+105 g_{30}{ }^{2} g_{10} g_{11} g_{20}+70 g_{20}{ }^{3} g_{30} g_{11}+45 g_{11} g_{10}{ }^{3} g_{60}\right) .
\end{aligned}
$$

If $g_{61}$ is obtained from the equation $L_{3}=0$, then

$$
\begin{aligned}
L_{4}= & \frac{-\pi}{17280\left(g_{10}\right)^{21 / 2}}\left(945 g_{11} g_{10}{ }^{5} g_{80}+2835 g_{20} g_{71} g_{10}{ }^{5}-4620 g_{20}{ }^{3} g_{51} g_{10}{ }^{3}+3080 g_{20}{ }^{5} g_{31} g_{10}\right. \\
& +1701 g_{30}{ }^{2} g_{11} g_{40} g_{10}{ }^{3}+8820 g_{30} g_{20}{ }^{3} g_{31} g_{10}{ }^{2}-1215 g_{30} g_{11} g_{60} g_{10}{ }^{4}-2835 g_{70} g_{10}{ }^{4} g_{11} g_{20} \\
& -2835 g_{30} g_{20} g_{51} g_{10}{ }^{4}-1701 g_{30} g_{40} g_{31} g_{10}{ }^{4}-8820 g_{30}{ }^{2} g_{10} g_{20}{ }^{3} g_{11}+4620 g_{20}{ }^{3} g_{50} g_{10}{ }^{2} g_{11} \\
& +1701 g_{40} g_{10}{ }^{5} g_{51}+5670 g_{30} g_{50} g_{10}{ }^{3} g_{11} g_{20}+4158 g_{30} g_{10}{ }^{2} g_{20}{ }^{2} g_{40} g_{11}-945 g_{81} g_{10}{ }^{6}-3080 g_{20}{ }^{5} g_{30} g_{11} \\
& +2835 g_{30}{ }^{2} g_{20} g_{31} g_{10}{ }^{3}-2835 g_{30}{ }^{3} g_{10}{ }^{2} g_{11} g_{20}-2835 g_{50} g_{20} g_{31} g_{10}{ }^{4}-1701 g_{50} g_{11} g_{40} g_{10} 4 \\
& \left.-4158 g_{20}{ }^{2} g_{40} g_{31} g_{10}{ }^{3}+1215 g_{60} g_{10}{ }^{5} g_{31}\right) .
\end{aligned}
$$

It should be noted that the expressions for the subsequent Lyapunov values of Lienard system [Leonov \& Kuznetsova, 2009] and the expressions for Lyapunov values of general systems [Kuznetsov, 2008], which are obtained by computer, are so far complicated, that they can be used only in the creation of the corresponding software libraries.

\subsubsection{Lyapunov values and small limit cycles in quadratic systems}

Remark that in the general case, if $L_{1, \ldots, n-1}=0$ and $L_{n} \neq 0$, then, using the well-known Bautin technique [Bautin, 1952], $n$ small limit cycles can be constructed by small perturbation of system coefficients (see, e.g. [Lynch, 2010]).

Consider formula (41)

$$
y(T(h), h)-h=L_{0} h+L_{1} h^{3}+\cdots,
$$

and suppose that $L_{0}=0$ and the first nonzero Lyapunov value $L_{1}>0$. Then, using a dependence of $L_{i}$ on coefficients of the considered system by Bautin's technique [Bautin, 1952] (with the help of small perturbation of coefficients of the considered system), an effort can be made to satisfy the inequalities

$$
L_{0}<0, \quad L_{1}>0, \quad\left|L_{0}\right| \ll\left|L_{1}\right|
$$

for the perturbed system. For example, the possibility of such sequential perturbations is easily observed for the Lyapunov values of general Lienard system, which are given above - in the expression for $L_{i}$ there is a unique addend with $g_{2 i, 1}$ in the first degree. 
In this case, for sufficiently small initial data $h=y_{0}{ }^{I}$ the trajectories of the perturbed system are wound around a stationary point, while for certain initial data $h=y_{0}{ }^{I I}\left(y_{0}{ }^{I I} \gg y_{0}{ }^{I}\right)$ the trajectories of the system are unwound. Thus, for such perturbations, a "small" unstable limit cycle can be obtained around zero equilibria.

Similarly, perturbating a few first Lyapunov values, due to the smallness of perturbations and the continuous dependence of solutions on parameter in the perturbed system, a few "small" limit cycles can be obtained.

However, for specific systems when the number of considered coefficients is bounded, the question arises whether the independent perturbations of sequential Lyapunov values are possible (the question, which till now is not solved in the general case). Illustrate this on the example of quadratic systems. Suppose that at the point of equilibrium $x=y=0$, a matrix of linear approximation of reduced quadratic system (11) has two purely imaginary eigenvalues, that is, $L_{0}(0)=0$ (weak focus of at least first order). Then the following relations

$$
\alpha<0, \quad \beta=0: L_{0}(0)=0
$$

are satisfied.

By the reduction of quadratic system to Lienard system with functions (14) and the obtained expressions for Lyapunov values, for system (11), the expressions for Lyapunov values are obtained. The first Lyapunov value is as follows

$$
L_{1}(0)=\frac{-\pi}{4(-\alpha)^{5 / 2}}(\alpha(b c-1)-a(b+2)) .
$$

Determine the conditions under which $L_{1}(0)=$ $0, L_{2,3}(0) \not \equiv 0$. From the above, we get

$$
\begin{aligned}
& \alpha=\frac{a(2+b)}{b c-1}<0, \quad \beta=0 \\
& \begin{aligned}
L_{2}(0)= & \frac{\pi}{24(-\alpha)^{7 / 2}} \frac{(b-3)}{(b c-1)}((c b+b-2 c)(c b-1) \\
& \left.-a(c-1)(1+2 c)^{2}\right)
\end{aligned}
\end{aligned}
$$

or

$$
\begin{aligned}
& b c=1, \quad a=0, \quad \alpha<0, \quad \beta=0 \\
& L_{2}(0)=\frac{\pi}{24(-\alpha)^{7 / 2}} \frac{(b-1)(b-3)(2+b)\left(b^{2}+\alpha\right)}{b^{3}} .
\end{aligned}
$$

Then the equilibrium $x=0, y=0$ of system (11) is a weak focus of at least second order (if $b=-2$ and $c=-1 / 2$, then $\left.L_{2}=L_{3} \equiv 0\right)$.

Determine the conditions under which $L_{1}(0)=$ $L_{2}(0)=0, L_{3}(0) \not \equiv 0$. This results in

$$
\begin{aligned}
& b=3, \quad \alpha=\frac{a(2+b)}{b c-1}<0, \quad \beta=0 \\
& L_{3}= \frac{\pi}{160(-\alpha)^{9 / 2}}(c-2)\left((c-1)(1+2 c)^{2} a\right. \\
&-(c+3)(3 c-1))
\end{aligned}
$$

or

$$
\begin{aligned}
& b=\sqrt{-\alpha}, \quad b c=1, \quad a=0, \quad \alpha<0, \quad \beta=0 \\
& L_{3}(0)=-\frac{\pi}{4608(-\alpha)^{17 / 2}}(\alpha+2)\left(\alpha^{2}-16\right)(\alpha+6) \\
& \quad \times\left(67 \alpha^{2}-614 \alpha+964\right) .
\end{aligned}
$$

If system coefficients are chosen so that $L_{1,2,3}=0$, then $L_{4,5, \ldots}=0$.

For quadratic system, this technique allows one to construct three nested small limit cycles $(C(2)=$ 3 ) in the case when the coefficients of the system are chosen so that $L_{1,2}=0$ and $L_{3} \neq 0$ (a weak focus of third order) or to construct two small limit cycles one around each of two weak focuses of first order [Leonov, 2011].

For a cubic system, 11 small limit cycles at one point can be constructed $(C(3) \geq 11)$ [Żołądek, 1995], and in general $C(n) \geq n^{2}-1$ for even $n$ [Qiu \& Yang, 2009]. An approach to get an upper bound for $C(n)$ is discussed in [Giné, 2009].

Note also that the conditions (62) and (64) are not compatible with the conditions of Theorem 2 .

\subsection{Large and small limit cycles}

Consider the main results on configuration and maximal number of limit cycles in quadratic system. The distribution of limit cycles of quadratic systems has only one or two nests. At least one of the two nests contains only unique limit cycle [Zhang, 2002].

In quadratic system there are no limit cycles around a degenerated focus of third order [Li, 1986; Cherkas, 1986]. Nowadays a widely-known conjecture is that in quadratic system the maximum possible number of limit cycles is $4(H(2)=4)$ but to date a rigorous proof of this hypothesis is absent. 
At present, in the frame of the proof of this hypothesis, investigations are performed concerning the systematization and analysis of various cases of qualitative behavior in quadratic systems, but these investigations are yet to be completed (see [Artes \& Llibre, 1997; Schlomiuk \& Pal, 2001; Schlomiuk \& Vulpe, 2005; Artes et al., 2006, 2008]).

Let us proceed to the visualization of limit cycles in quadratic systems with weak focuses, using the above results on a global behavior of trajectories and the local analysis of weak focuses.

Recall that relations (28) and (30) yield the inequalities $b>-1, a<0$. Then, taking into account (61), one can obtain

$$
b c>1
$$

and therefore by (28)

$$
b>1 .
$$

In the case of weak focus the second Lyapunov value $L_{2}(0)$ is positive for $b<3$ or in view of (66)

$$
b \in(1,3) .
$$

Hence by (65) $c$ satisfies the inclusion

$$
c \in\left(\frac{1}{3}, 1\right)
$$

and condition (31) is valid. Note that by (67) and (68) the first inequality in (30) is satisfied.

Thus, the conditions of Propositions 5 and 7 are satisfied and at zero point there is a weak focus. Then $g(x)$ has only two zeros $x=0$ and $x_{1}<-1$ and both equilibria of system (11) are unstable. In this case, by Theorem 4 , if relations (30), (61), (65), (67), and (68) are satisfied, then system (11) has two limit cycles.

For small perturbation of the parameters

$$
\begin{aligned}
& \beta \in(0, \varepsilon) \\
& \alpha \in\left(\frac{a(2+b)}{b c-1}, \frac{a(2+b)}{b c-1}+\delta\right),
\end{aligned}
$$

where $0<\varepsilon \ll \delta \ll 1$, two large limit cycles persists and two additional small limit cycles in the neighborhood of zero are born. Thus, if conditions (30), (65), (67), (68), and (69) are satisfied, system (11) has four limit cycles (two small and two large). The domain, defined by these conditions, has an infinite Lebesgue measure. However this domain is small with respect to parameters $\beta$ and $\alpha$.
Note that the domain of unperturbed parameters is three-dimensional. It has the form

$$
\begin{gathered}
\left\{b \in(1,3), c \in\left(\frac{1}{3}, 1\right), b c>1,\right. \\
\left.a(c-1)>\frac{(b-1)^{2}}{4}\right\} .
\end{gathered}
$$

In Fig. 14 are shown two large limit cycles (additional two small cycles at zero point can be obtained by small perturbations of parameters of system (11)). Here system coefficients are the following

$$
\begin{gathered}
a=-35, \quad b=1.6, \quad c=0.7, \\
\alpha=-1050, \quad \beta=0
\end{gathered}
$$

and in the domain of closeness of trajectories, one can see one stable (on the right) and one unstable (on the left) limit cycles.

In the limit case $b=3$, one gets $L_{2}=0$ and by (63) $L_{3}$ is negative for all $a$ and $c$, satisfying (30) and (68). It means that by a small positive perturbation $\mu$

$$
b \in(3-\mu, 3),
$$

the condition $L_{2}>0$ can be satisfied and the third small limit cycle at zero point can be obtained.

Note that in the case $b=3$, condition (65) is satisfied if (68) is taken into account. The results obtained mean that system (11) has four limit cycles (three small at zero point and one large at the point $\left.x_{1}<-1\right)$ if (30), (71), (68), and (69) are satisfied. Here $1 \gg \mu \gg \delta \gg \varepsilon \geq 0$. The domain of unperturbed parameters, corresponding to the conditions described above, is two-dimensional. It has the form

$$
\left\{c \in\left(\frac{1}{3}, 1\right), a(c-1)>1\right\} .
$$

This domain involves entirely the domain, defined by famous Shi's theorem [Shi, 1980].

\subsection{Nonlocal theory on the existence of nested large limit cycles in quadratic system}

The above stated method of asymptotic integration of Lienard system permits one to obtain the existence criteria of two large limit cycles and the further development of this method to formulate an existence criterium of three large cycles. 


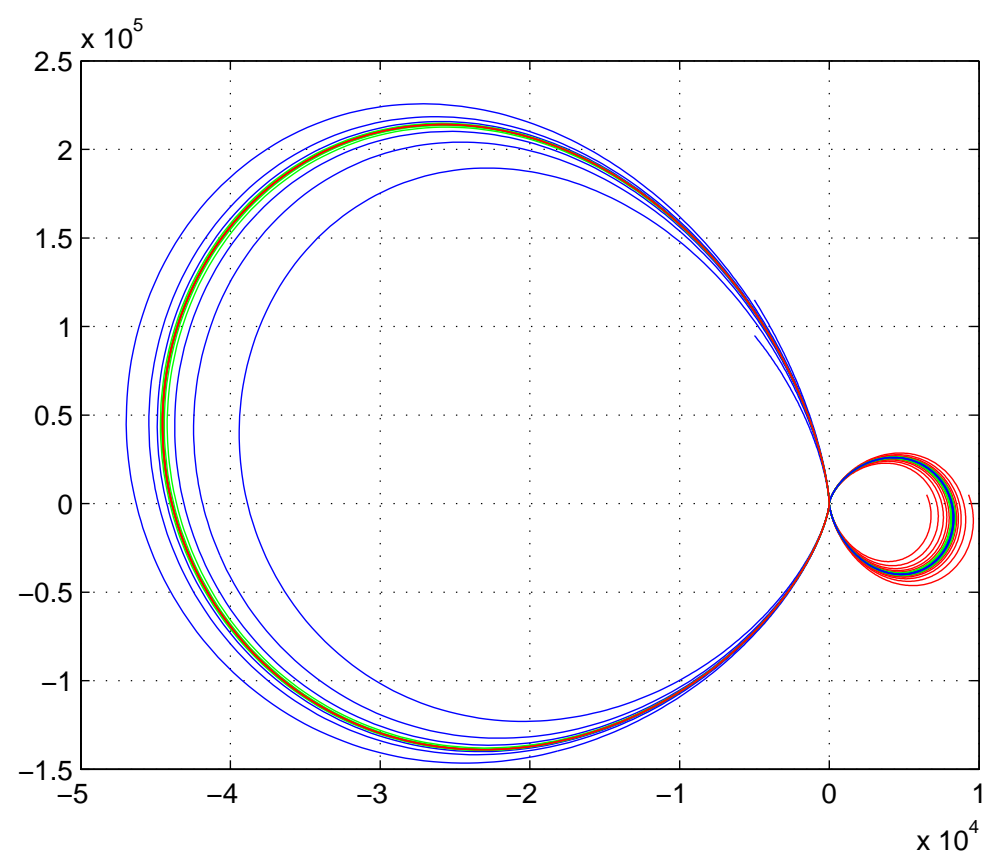

Fig. 14. Two large limit cycles.

Consider system (11). Suppose that the relations

$$
\begin{gathered}
c \in\left(\frac{1}{3}, 1\right), \quad c \neq \frac{1}{2} \quad \alpha=-\varepsilon^{-1}, \\
b>a+c, \quad 2 c<b+1, \\
4 a(c-1)>(b-1)^{2}
\end{gathered}
$$

are satisfied, where $\varepsilon$ is a small positive parameter.

Theorem 6 [Leonov, 2011]. Let system (11) have asymptotically stable equilibrium $x=y=0$. Then for sufficiently small $\varepsilon$, system (11) has three limit cycles: one of them is situated to the left of straight line $\left\{x=-1, y \in \mathbb{R}^{1}\right\}$ and two to the right of this straight line.

Proof. Below a sketch of the proof is given. Since the number $\varepsilon$ is sufficiently small, condition (31) is satisfied. Since inequalities (28) and (30) are valid, the behavior of the trajectories is as shown in Fig. 11. It follows the existence of limit cycle to the left of the straight line $\left\{x=-1, u \in \mathbb{R}^{1}\right\}$.

Show that to the right of this straight line, there is the trajectory $\gamma$, a behavior of which is shown in Fig. 15. From Fig. 15, here there are two limit cycles: one is between the trajectories $\Gamma$ and $\gamma$, the second limit cycle is between the trajectories $\gamma$ and $\rho$.
For the proof of the existence of such trajectory $\gamma$, further development of asymptotic integration method will be given. Consider the first order equation

$$
F \frac{d F}{d x}+f(x) F+g(x)=0 .
$$

This equation is equivalent to system (12). Having performed the change $F=\sqrt{-\alpha} G$, we obtain

$$
G \frac{d G}{d x}+\frac{f(x)}{\sqrt{-\alpha}} G+\frac{g(x)}{-\alpha}=0 .
$$

Further, the solution of Eq. (75) is considered with the initial data $G(0)=R=\varepsilon^{-0.01}$.

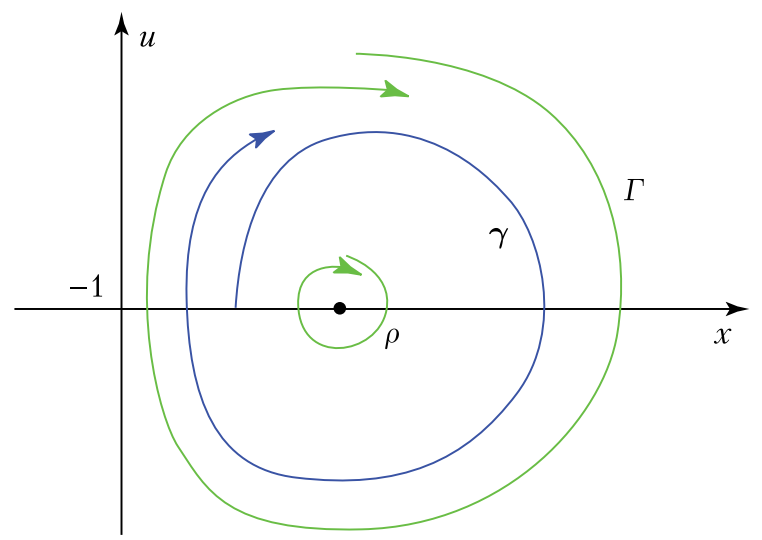

Fig. 15. 
1. Case $c \in(\mathbf{1} / \mathbf{2}, \mathbf{1})$. By the change $z=(x+1)^{k}$, $k=q+1 / 2$, Eq. (75) is reduced to the form

$$
\begin{aligned}
G d G & +\frac{f\left(z^{\frac{1}{k}}-1\right)}{k \sqrt{-\alpha}} z^{\frac{1}{k}-1} G d z \\
& +\frac{g\left(z^{\frac{1}{k}}-1\right)}{k(-\alpha)} z^{\frac{1}{k}-1} d z=0 .
\end{aligned}
$$

For $z \in[\nu R, 2 R]$, where $\nu=\varepsilon^{0.001}$, the following relation occurs

$$
\begin{aligned}
& \frac{g\left(z^{\frac{1}{k}}-1\right)}{(-\alpha)} z^{\frac{1}{k}-2}=1+O\left(\varepsilon^{0.009 / k}\right) \\
& f\left(z^{\frac{1}{k}}-1\right) z^{-\frac{q}{k}}=2 c-b-1+O\left(\varepsilon^{0.009 / k}\right) .
\end{aligned}
$$

By the change $z=(x+1)^{q}$, Eq. (75) becomes

$$
\begin{aligned}
G d G & +\frac{f\left(z^{\frac{1}{q}}-1\right)}{q \sqrt{-\alpha}} z^{\left(\frac{1}{q}-1\right)} G d z \\
+ & \frac{g\left(z^{\frac{1}{q}}-1\right)}{q(-\alpha)} z^{\left(\frac{1}{q}-1\right)} d z=0 .
\end{aligned}
$$

For $z \in[\nu R, 2 R]$,

$$
\begin{aligned}
& \frac{g\left(z^{\frac{1}{q}}-1\right)}{(-\alpha)} z^{-2+\frac{1}{q}}=-1+O\left(\varepsilon^{0.009 /(-q)}\right), \\
& f\left(z^{\frac{1}{q}}-1\right) z^{-1+\frac{2}{q}}=1+2 c+O\left(\varepsilon^{0.009 /(-q)}\right) .
\end{aligned}
$$

Equations (76) and (78) are equivalent to the second order equations

$$
\ddot{z}+\frac{f\left(z^{\frac{1}{k}}-1\right)}{k \sqrt{-\alpha}} z^{\frac{1}{k}-1} \dot{z}+\frac{g\left(z^{\frac{1}{k}}-1\right)}{k(-\alpha)} z^{\frac{1}{k}-1}=0
$$

and

$$
\ddot{z}+\frac{f\left(z^{\frac{1}{q}}-1\right)}{q \sqrt{-\alpha}} z^{\frac{1}{q}-1} \dot{z}+\frac{g\left(z^{\frac{1}{q}}-1\right)}{q(-\alpha)} z^{\frac{1}{q}-1}=0,
$$

respectively. By (77) and (79), one obtains that an approximation of solutions, with respect to $\varepsilon$, of these equations with the initial data $z(0)=0$, $\dot{z}(0)=R$ are the following functions

$$
\begin{aligned}
& z_{1}(t)=\frac{R}{\omega_{1}} \sin \omega_{1} t \quad \omega_{1}=\frac{1}{\sqrt{k}}, \\
& z_{2}(t)=\frac{R}{\omega_{2}} \sin \omega_{2} t \quad \omega_{2}=\frac{1}{\sqrt{-q}} .
\end{aligned}
$$

For Eqs. (80) and (81), we consider Lyapunov type functions

$$
V_{1}=\left(\dot{z}_{1}\right)^{2}+\int_{0}^{z} \frac{g\left(y^{\frac{1}{k}}-1\right)}{k(-\alpha)} y^{\frac{1}{k}-1} d y
$$

and

$$
V_{2}=\left(\dot{z}_{2}\right)^{2}+\int_{0}^{z} \frac{g\left(y^{\frac{1}{q}}-1\right)}{q(-\alpha)} y^{\frac{1}{q}-1} d y,
$$

respectively. For these functions the following relations

$$
\begin{aligned}
& \dot{V}_{1}(t)=-2 \frac{f\left(z^{\frac{1}{k}}-1\right)}{k \sqrt{-\alpha}} z^{\frac{1}{k}-1}(\dot{z})^{2} \\
& \dot{V}_{2}(t)=-2 \frac{f\left(z^{\frac{1}{q}}-1\right)}{q \sqrt{-\alpha}} z^{\frac{1}{q}-1}(\dot{z})^{2}
\end{aligned}
$$

are satisfied.

Relation (83) is valid for the solutions of Eq. (80), relation (84) for those of (81).

From (77), (82), (83) or from (79), (82), (84) it follows that for small $\varepsilon$ on the intervals $\left[0, \pi / \omega_{1}\right]$ and $\left[0, \pi / \omega_{2}\right]$, the increments of the functions $V_{1}$ and $V_{2}$ along the solutions of Eqs. (80) and (81) with the initial data $z(0)=0, \dot{z}(0)=R$ have the following asymptotic

$$
\begin{aligned}
V_{1}\left(\frac{\pi}{\omega_{1}}\right)-V_{1}(0) \approx & -\frac{2 k^{-\frac{q}{2 k}}(2 c-b-1)}{\sqrt{-\alpha}} R^{2+\frac{1}{2 k}} \\
& \times \int_{0}^{\pi}(\sin \tau)^{\frac{1}{2 k}}(\cos \tau)^{2} d \tau, \\
V_{2}\left(\frac{\pi}{\omega_{2}}\right)-V_{2}(0) \approx & \frac{2\left(-q^{\frac{1+q}{-2 q}}\right)(1+2 c)}{\sqrt{-\alpha}} R^{2-\frac{1}{q}} \\
& \times \int_{0}^{\pi}(\sin \tau)^{-\frac{1}{q}}(\cos \tau)^{2} d \tau .
\end{aligned}
$$

Since $R=\varepsilon^{-0.01}, 2 k=2 q+1<-q$, for small $\varepsilon$ the inequality

$$
V_{1}\left(\frac{\pi}{\omega_{1}}\right)-V_{1}(0)>R^{\frac{3 c-1}{2 c(1-2 c)}}\left(V_{2}\left(\frac{\pi}{\omega_{2}}\right)-V_{2}(0)\right)
$$

is satisfied.

2. Case $c \in(\mathbf{1} / \mathbf{3}, \mathbf{1} / \mathbf{2})$. Make the following change $z=(x+1)^{1-c}, x>0$. In this case, Eq. (75) can be represented as

$$
\begin{aligned}
G d G & +\frac{f\left(z^{1 /(1-c)}-1\right)}{(1-c) \sqrt{-\alpha}} z^{\frac{c}{1-c}} G d z \\
& +\frac{g\left(z^{1 /(1-c)}-1\right)}{(1-c)(-\alpha)} z^{\frac{c}{1-c}} d z=0 .
\end{aligned}
$$




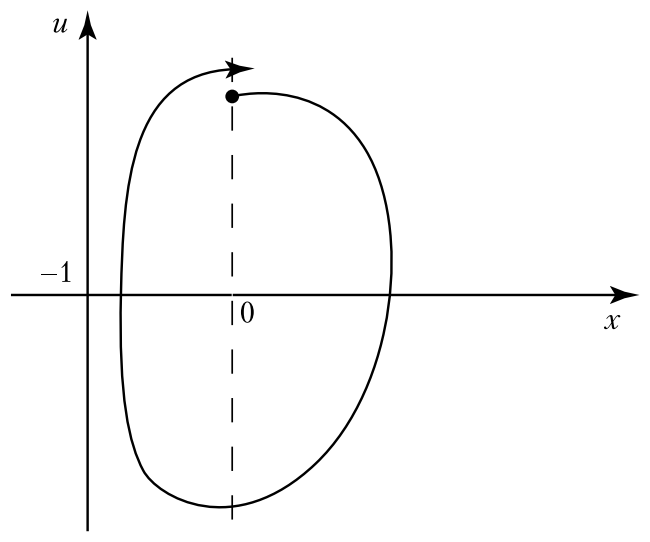

Fig. 16. Trajectory $\gamma$.

For $z \geq \nu R$, the estimates

$$
\begin{aligned}
\frac{g\left(z^{1 /(1-c)}-1\right)}{-\alpha} z^{\frac{c}{1-c}}= & z^{-\frac{c}{1-c}}+\varepsilon(b-a-c) z \\
& +O(\varepsilon) z^{-\frac{c}{1-c}} \\
f\left(z^{1 /(1-c)}-1\right) z^{\frac{c}{1-c}}= & (2 c-b-1)+O\left(\varepsilon^{0.09 / 1-c}\right)
\end{aligned}
$$

are satisfied.

In this case for the solution of Eq. (85) with $G(1)=R$ the following asymptotic estimates

$$
\begin{aligned}
G(z)^{2} & \approx R^{2}-\frac{2}{2 c-1}\left(1-z^{\frac{1-2 c}{1-c}}\right)-\frac{2 \varepsilon(b-a-c)}{(1-c)} z^{2} \\
& \approx R^{2}-\frac{2 \varepsilon(b-a-c)}{(1-c)} z^{2}
\end{aligned}
$$

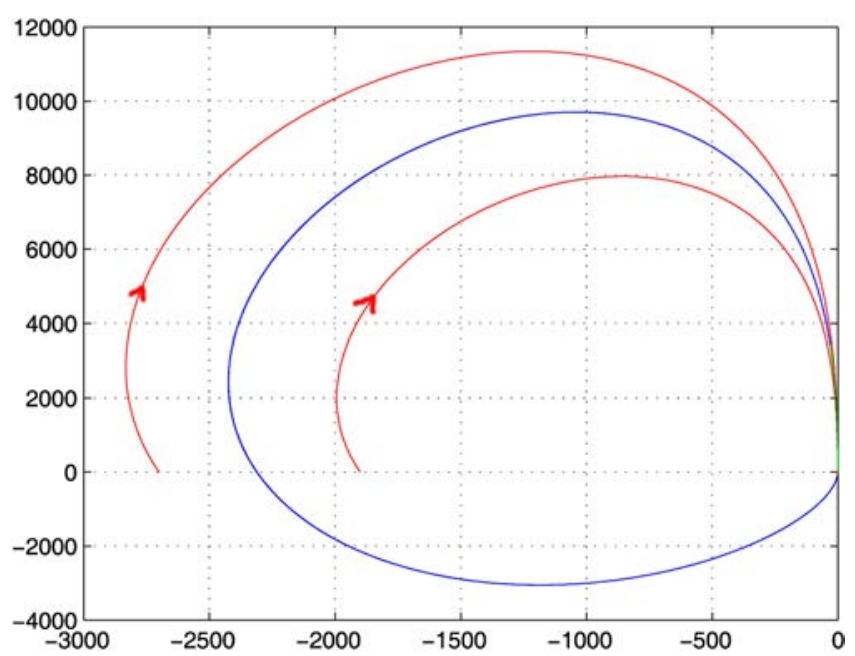

are valid. This implies that for the difference $G(1)^{2}-R^{2}$ the relation

$$
\begin{aligned}
G(1)^{2}-R^{2} \approx & \int_{1}^{z_{0}} \frac{2(-2 c+b+1)}{(1-c) \sqrt{-\alpha}} \\
& \times \sqrt{R^{2}-\frac{2 \varepsilon(b-a-c) z^{2}}{(1-c)}} d z,
\end{aligned}
$$

where

$$
z_{0}=R \sqrt{\frac{1-c}{2 \varepsilon(b-a-c)}}, \quad(G(1)<0)
$$

is satisfied. Then

$$
G(1)^{2}-R^{2} \approx \frac{\pi(-2 c+b+1) R^{2}}{\sqrt{2}(1-c)(b-a-c)}, \quad G(1)<0 .
$$

Apply the reasoning of Case 1 to the solution of Eq. (76) with $G(1)=R$. Then

$$
\begin{aligned}
G(1)^{2}-R^{2} \approx & \frac{2\left(c^{\frac{1-c}{2 c}}\right)(1+2 c)}{\sqrt{-\alpha}} R^{2+\frac{1}{c}} \\
& \times \int_{0}^{\pi}(\sin \tau)^{\frac{1}{c}}(\cos \tau)^{2} d \tau, \quad G(1)<0 .
\end{aligned}
$$

From the estimates, obtained in Cases 1 and 2, it follows that a behavior of trajectory of system (12) with initial data $x(0)=0, u(0)=R \sqrt{-\alpha}$, is the same as $\gamma$ shown in Fig. 16.

This establishes Theorem 6 .

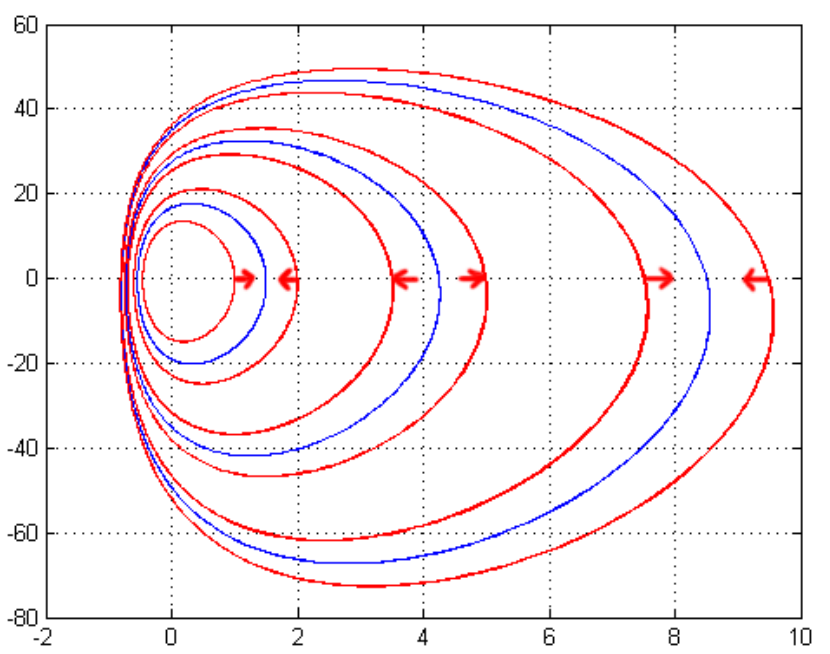

Fig. 17. Visualization of four limit cycles [Kuznetsov et al., 2013]. 
Below, it will be demonstrated that an additional perturbation of zero equilibria, permits one to obtain here four limit cycles.

\subsection{Solution of Kolmogorov's problem. Visualization of four limit cycles}

For the visualization of four limit cycles in quadratic system, we use the values of parameters of the system from set (70). At first, perturbing $\alpha$, the third limit cycle can be obtained and then, by perturbing $\beta$, the fourth limit cycle.

For the set of coefficients $b=2.7, c=0.4$, coefficients $a=-10, \alpha=-437.5, \beta=0.003$ are selected. In this case, three large limit cycles are observed around zero point and one large limit cycle to the left of the straight line $x=-1$ (Fig. 17).

\section{Hidden Oscillations in Applied Models}

In applied systems, the finding of hidden oscillations, which cannot be detected by standard simulation, has shown that for them to be studied, it is necessary to develop special effective methods [Lauvdal et al., 1997]: "Since stability in simulations does not imply stability of the physical control system (an example is the crash of the YF22 [Boeing]), stronger theoretical understanding is required." Consider a few examples of such systems.

\subsection{Phase-locked-loop circuits}

The phase-locked loop (PLL) systems were invented in the 1930s-1940s [Bellescize, 1932; Wendt \& Fredentall, 1943] and were widely used in radio and television (demodulation and recovery, synchronization and frequency synthesis). Nowadays, PLL can be produced in the form of single integrated circuit and the various modifications of PLL are used in a variety of modern electronic applications (radio, telecommunications, computers, and others). Various methods for the analysis of phaselocked loops have been well developed by engineers and are considered in many publications (see, e.g. [Viterbi, 1966; Gardner, 1966; Lindsey, 1972; Shakhgil'dyan \& Lyakhovkin, 1972]) but the problems of the construction of adequate nonlinear models and the nonlinear analysis of such models which are still far from being resolved, turn out to be difficult, and require to use special methods of the qualitative theory of differential, difference, integral, and integro-differential equations [Leonov et al., 1996; Suarez \& Quere, 2003; Margaris, 2004; Leonov, 2006; Kudrewicz \& Wasowicz, 2007; Leonov et al., 2009; Kuznetsov et al., 2011c; Leonov et al., 2011d, 2012b].

Below, it will be illustrated some difficulties arising in the analysis of comparatively simple nonlinear two-dimensional dynamical model of PLL.

In the middle of the last century, the investigations of dynamical models of phase synchronization systems were begun. Kapranov [1956] obtained the conditions of global stability for the following two-dimensional PLL model (with the filter of type $\left.W(p)=\frac{a p+\beta}{p+\alpha}\right)$

$$
\begin{aligned}
& \dot{z}=-\alpha z-(1-a \alpha)(\varphi(\sigma)-\gamma), \quad a, \alpha, \gamma \geq 0, \\
& \dot{\sigma}=z-a(\varphi(\sigma)-\gamma),
\end{aligned}
$$

where $\varphi(\sigma)$ is a $2 \pi$-periodic characteristic of the phase detector.

In 1961, Gubar' [1961] revealed a gap in the proof of Kapranov's results and indicated system parameters for which a semistable periodic solution can exist. Such a semistable trajectory cannot be found numerically by the standard computation procedure, thus from the computational point of view the system considered was globally stable (all the trajectories tend to equilibria), but, in fact, there is a bounded domain of attraction only.

Following [Leonov et al., 1996], the qualitative analysis of system (86) will be considered below.

Theorem 7. Any bounded in $\mathbb{R}^{2}$ for $t \in[0,+\infty)$ solution of system (86) tends to a certain equilibrium as $t \rightarrow+\infty$.

Further, without loss of generality, it is assumed that $-\min _{\sigma} \varphi(\sigma)=\max _{\sigma} \varphi(\sigma)=1$. Then from Theorem 7 it follows that for $\gamma>1$ (i.e. in the absence of equilibria) all the solutions of system (86) are unbounded. In this case, the synchronization does not occur under any initial conditions $z(0), \sigma(0)$.

Suppose that on the set $[0,2 \pi)$ there are exactly two zeros of the function $\varphi(\sigma)-\gamma: \sigma=\sigma_{1}, \sigma=\sigma_{2}$. Besides $\varphi^{\prime}\left(\sigma_{1}\right)>0, \varphi^{\prime}\left(\sigma_{2}\right)<0$.

Consider the case $\gamma<1$. In this case, in phase space, the curve $z=a(\varphi(\sigma)-\gamma)$ and on this curve 


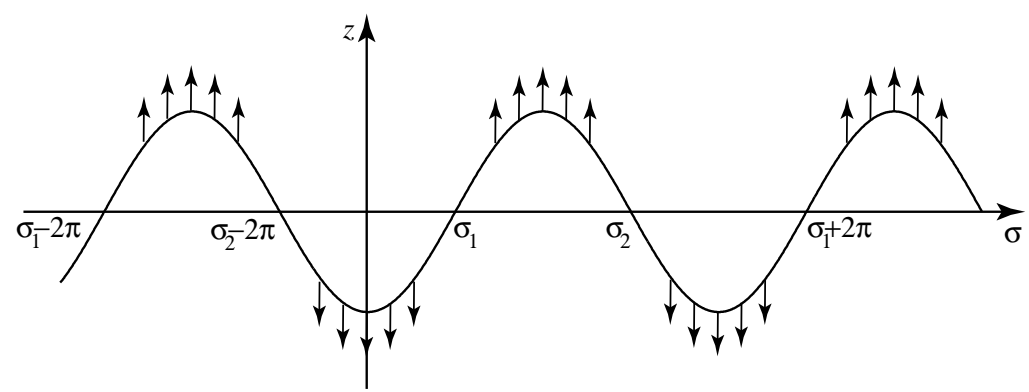

Fig. 18. Vector field on the curve $z=a(\varphi(\sigma)-\gamma)$.

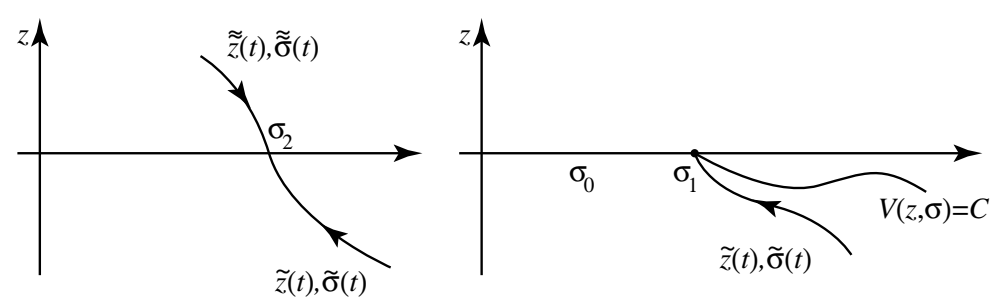

Fig. 19. Separatrices of saddle point.

equilibria $z=0, \sigma=\sigma_{0}$, where $\varphi\left(\sigma_{0}\right)=\gamma$ play an important role (Fig. 18).

Consider the equilibrium $z=0, \sigma=\sigma_{2}$. In the neighborhood of this point the characteristic polynomial of first approximation linear system is as follows

$$
p^{2}+\left(\alpha+a \varphi^{\prime}\left(\sigma_{2}\right)\right) p+\varphi^{\prime}\left(\sigma_{2}\right) .
$$

The inequality $\varphi^{\prime}\left(\sigma_{2}\right)<0$ implies that the characteristic polynomial (87) has one positive and one negative zero and the stationary point $z=0, \sigma=\sigma_{2}$ is a saddle.

In this case, only two trajectories of system (86) - the separatrices of saddle - tend to equilibrium $z=0, \sigma=\sigma_{2}$ as $t \rightarrow+\infty$. Denote them as $\tilde{z}(t), \tilde{\sigma}(t)$ and $\tilde{\tilde{z}}(t), \tilde{\tilde{\sigma}}(t)$ (Fig. 19).

The following assertion can be proved.

Theorem 8. The relations

$$
\begin{gathered}
\tilde{z}(t)<0, \quad \forall t, \\
\lim _{t \rightarrow-\infty} \tilde{z}(t)=-\infty, \quad \lim _{t \rightarrow-\infty} \tilde{\sigma}(t)=+\infty
\end{gathered}
$$

are satisfied.

The proof of this theorem is based on the consideration of Lyapunov function in the form $V(z, \sigma)=$ $z^{2} / 2+(1+a \alpha) \int_{0}^{\sigma}(\varphi(\theta)-\gamma) d \theta$

Consider now the behavior of separatrix $\tilde{\tilde{z}}(t)$, $\tilde{\tilde{\sigma}}(t)$. In this case, there are more opportunities of qualitative behavior, than those given by relations (88), (89) for the separatrix $\tilde{z}(t), \tilde{\sigma}(t)$.
Three cases are possible:

(1) There exists a number $\tau$ such that $\tilde{\tilde{z}}(\tau)=$ $a(\varphi(\tilde{\sigma}(\tau))-\gamma), \tilde{\tilde{\sigma}}(\tau) \in\left(\sigma_{2}-2 \pi, \sigma_{1}\right), \tilde{\tilde{z}}(t)>$ $a(\varphi(\tilde{\tilde{\sigma}}(t))-\gamma), \forall t \in(\tau,+\infty)$. In Fig. 20 are shown the separatrices of saddle $z=0, \sigma=\sigma_{2}$ and the curve $z=a(\varphi(\sigma)-\gamma)$.

(2) For all $t \in \mathbb{R}^{1}$, the relation $\tilde{\tilde{z}}(t)>0$ is satisfied (see Fig. 21) and

$$
\begin{aligned}
& \lim _{t \rightarrow-\infty} \tilde{\tilde{z}}(t)=0, \\
& \lim _{t \rightarrow-\infty} \tilde{\tilde{\sigma}}(t)=\sigma_{2}-2 \pi .
\end{aligned}
$$

(3) For all $t \in \mathbb{R}^{1}$, the relation $\tilde{\tilde{z}}(t)>0$ is satisfied (see Fig. 22) and

$$
\lim _{t \rightarrow-\infty} \tilde{\tilde{\sigma}}(t)=-\infty
$$

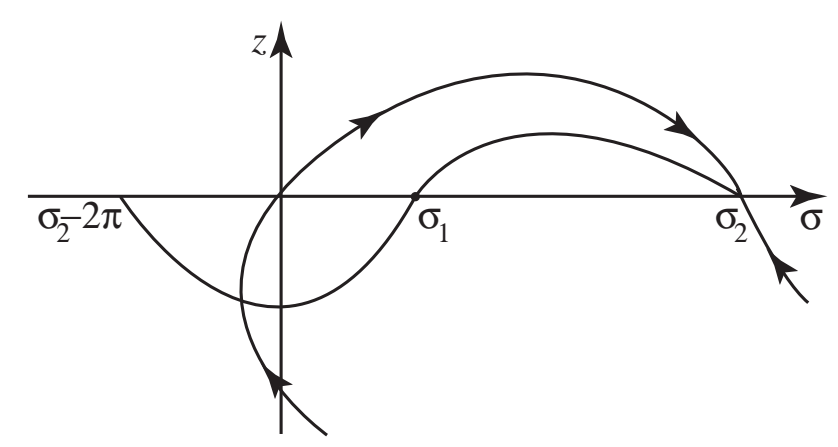

Fig. 20. Behavior of separatrix in Case 1. 


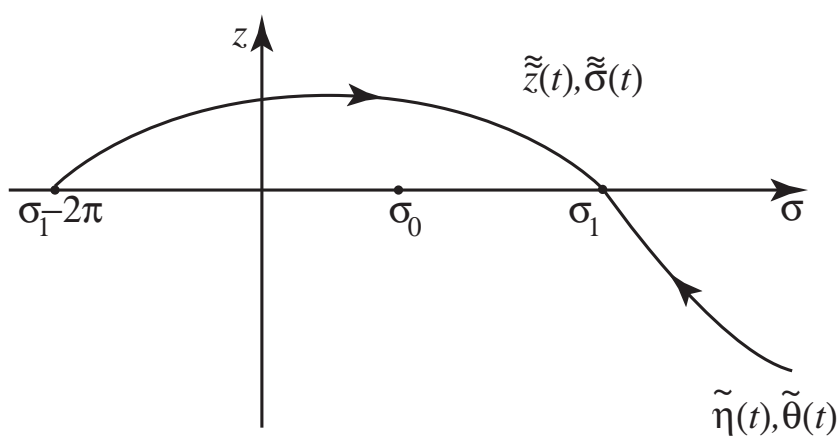

Fig. 21. Behavior of separatrix in Case 2.

In this case, there are two possibilities:

(3a) As $t \rightarrow-\infty$, the separatrix tends to infinity as the coordinate $z$ tends to infinity:

$$
\lim _{t \rightarrow-\infty} \tilde{\tilde{z}}(t)=+\infty .
$$

As was shown first by Tricomi [1933], this case always occurs for $a=0$.

(3b) As $t \rightarrow-\infty$, the separatrix remains bounded with respect to coordinate $z$ $\left(\overline{\lim }_{t \rightarrow-\infty} \tilde{\tilde{z}}(t) \leq\right.$ const $)$ and tends to the graph of a certain periodic function $\{z=$ $G(\sigma), G(\sigma+2 \pi)=G(\sigma)\}$ (Fig. 23). The curve $z=G(\sigma)$ corresponds to an unstable trajectory, to which the separatrices of saddles tend (from below) as $t \rightarrow-\infty$.

For $\varphi(\sigma)=\operatorname{sign} \sin (\sigma)$, this effect was discovered first in the work [Gubar', 1961]. In this case, the piecewise-linearity property of nonlinearity allows one to integrate a system on linearity intervals and then to apply Andronov's pointtransformation method to the investigation of limit cycle existence.

From Theorems 7 and 8 and the above stated properties of the separatrix of the saddle $\tilde{\tilde{z}}(t), \tilde{\tilde{\sigma}}(t)$, the conclusion can be made that for $\gamma \in[0,1]$,

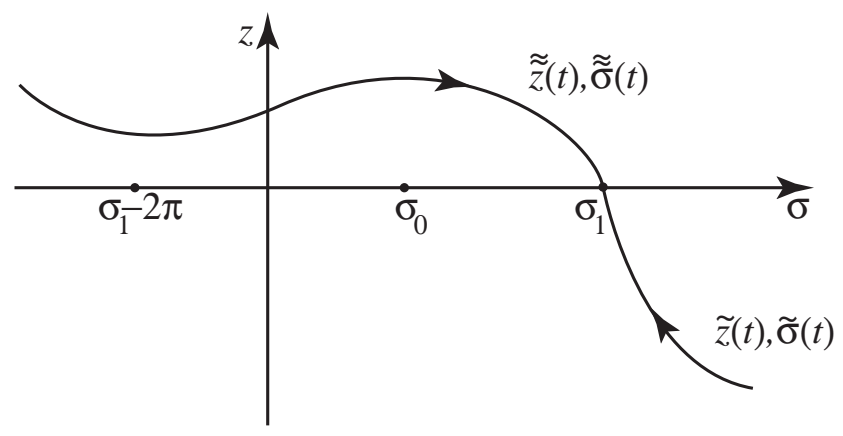

Fig. 22. Behavior of separatrix in Case 3.

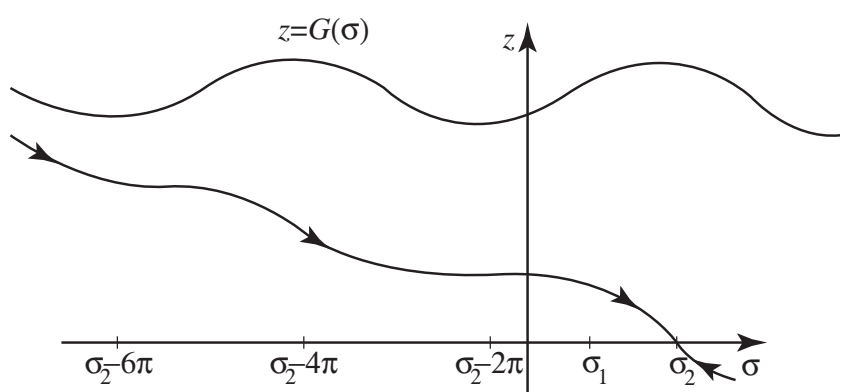

Fig. 23. Separatrices and the unstable cycle $z=G(\sigma)$ in Case 3b.

the following topologies of the phase space are possible:

(1) The separatrices $\tilde{z}(t), \tilde{\tilde{\sigma}}(t)$ and $\tilde{\tilde{z}}(t), \tilde{\tilde{\sigma}}(t)$ are the boundaries of the domains of attraction of locally asymptotically stable equilibrium $z=0$, $\sigma=\sigma_{1}$ (Fig. 24). In the displacement along $\sigma$ by $2 \pi$ these domains become the domains of attraction of stationary solutions $z=0$, $\sigma=\sigma_{1}+2 k \pi$.

The trajectories, placed outside these domains bounded by separatrices, tend to infinity as $t \rightarrow+\infty$.

(2) The separatrix $\tilde{\tilde{z}}(t), \tilde{\tilde{\sigma}}(t)$ is a heteroclinic trajectory in $\mathbb{R}^{2}$, i.e.

$$
\begin{aligned}
& \lim _{t \rightarrow-\infty} \tilde{\tilde{z}}(t)=\lim _{t \rightarrow+\infty} \tilde{\tilde{z}}(t)=0, \\
& \lim _{t \rightarrow+\infty} \tilde{\tilde{\sigma}}(t)=\sigma_{2}, \quad \lim _{t \rightarrow-\infty} \tilde{\tilde{\sigma}}(t)=\sigma_{2}-\pi .
\end{aligned}
$$

In this case the attraction domains of asymptotically stable equilibria are also bounded by separatrices but in the semiplane $\{z \leq 0\}$ the unstable "corridors" are absent (Fig. 24).

(3) The phase space is partitioned into the domains of attraction of stable equilibria (this corresponds to Case 3a - see Fig. 25). The boundaries of these domains are separatrices of saddle equilibria (Fig. 26).

(4) The phase space is partitioned into the domains of attraction of stable equilibria with the boundaries, consisting of separatrices of saddles, and a domain, placed above the curve $\{z=G(\sigma)\}$. In this domain, all trajectories tend to infinity as $t \rightarrow+\infty$. This corresponds to Case 3b (Fig. 27).

It is clear that under any initial data $z(0)$, $\sigma(0)$ the synchronization corresponds to the global asymptotic stability of system (86). This property corresponds to Case 3 (Fig. 26). 


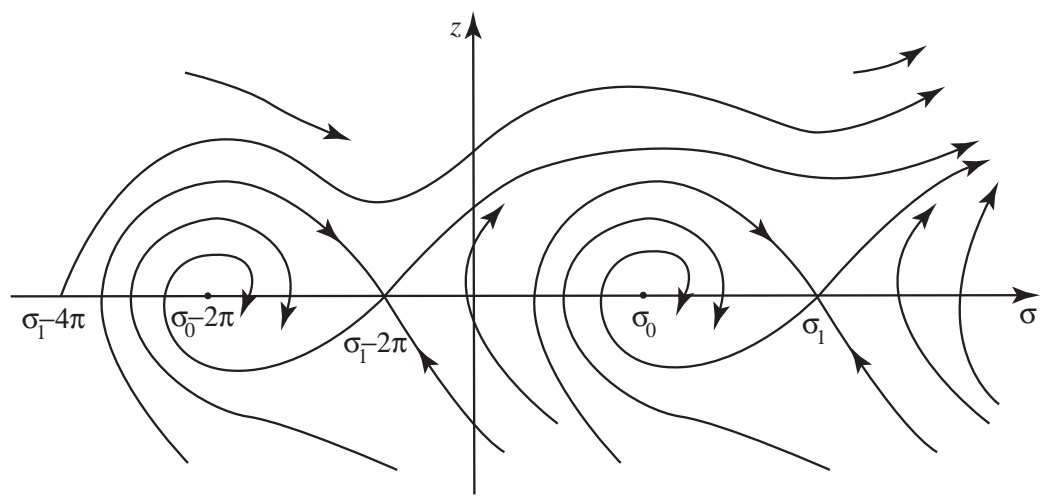

Fig. 24. The attraction domains bounded by separatrices.

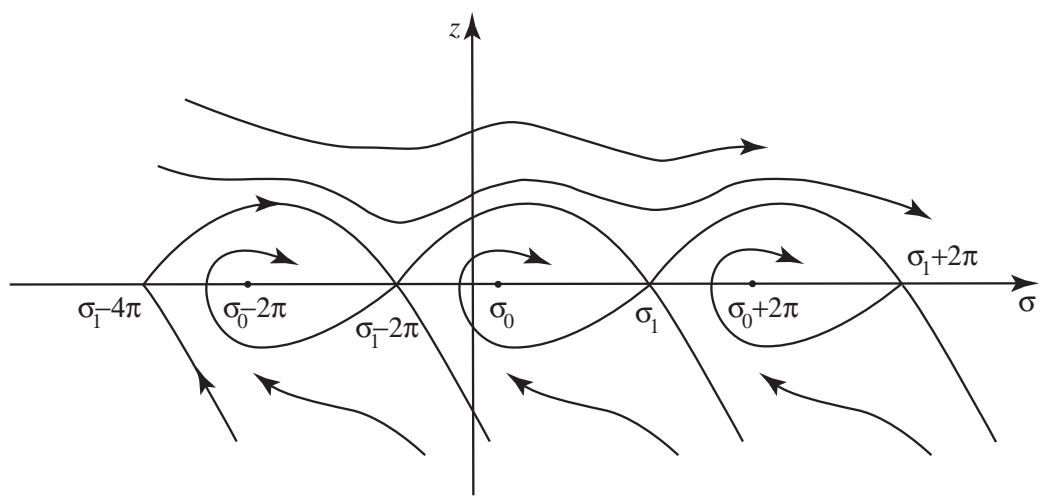

Fig. 25. Bifurcation of heteroclinic trajectory.

The loss of stability because of continuous dependence of trajectories with respect to system parameters can be caused only by two global bifurcations: the appearance of heteroclinic trajectory (Case 2, Fig. 21) or the appearance of semistable trajectory of the form $\{z=G(\sigma)\}$ (so-called second order cycle) (Case 4, Fig. 28). Further, one can consider bifurcation of hidden oscillation: stable and unstable periodic trajectories are bifurcated from the semistable periodic trajectories. If stable and unstable periodic solutions are very close to one another, then from a computational point of view, all the trajectories tend to equilibria, but, in fact, there is a bounded domain of attraction only.

Approximate computation of the bifurcation curves for the considered model was in [Belyustina et al., 1970] by the use of the above-described qualitative-numerical method.

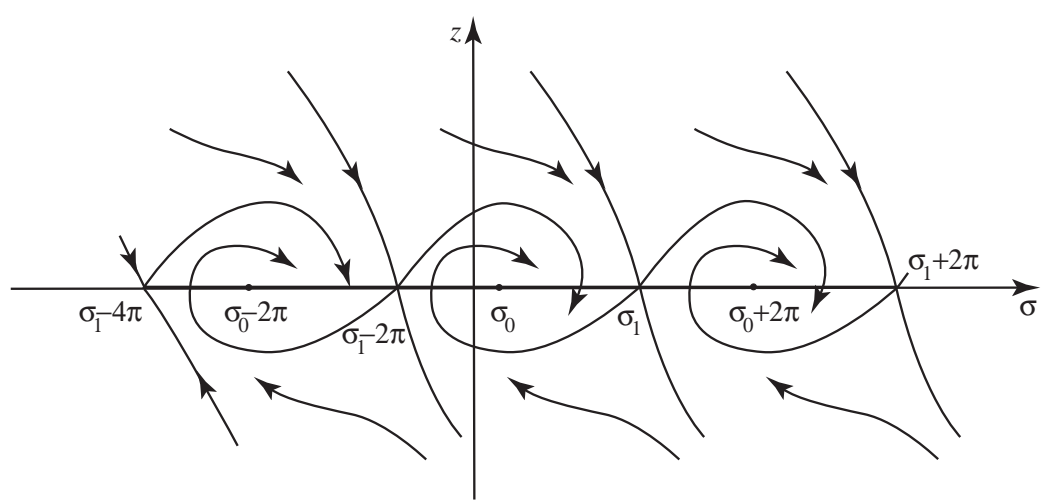

Fig. 26. Global asymptotic stability. 


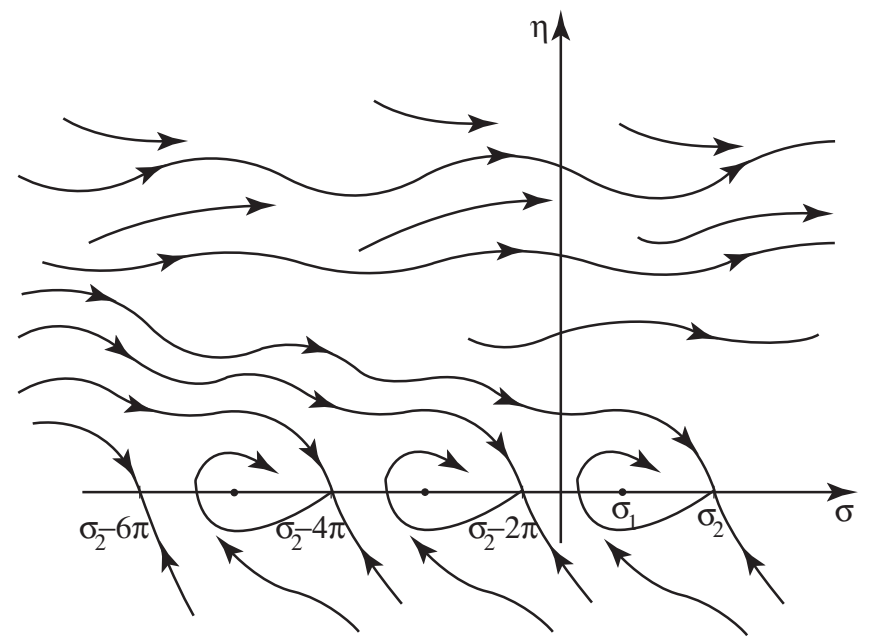

Fig. 27. Stability domains bounded by the cycle $z=G(\sigma)$.

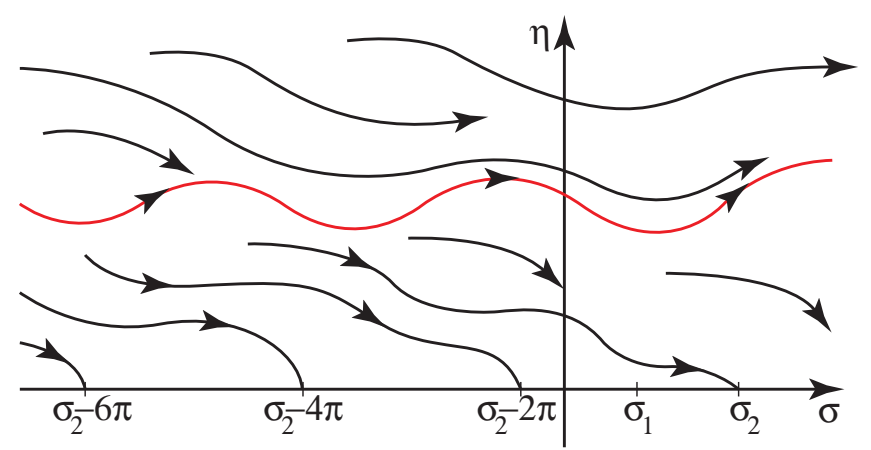

Fig. 28. Semistable periodic trajectory: bifurcation of hidden oscillation. From a computational point of view, all the trajectories tend to equilibria, but, in fact, there is a bounded domain of attraction only.

\subsection{Electrical machines}

Despite there being a lot of models describing drilling systems, drill string failures, which cause enormous cost losses for the drilling industry, still occur. Here, following [Kiseleva et al., 2012], on the examples of a two-mass model [de Bruin et al., 2009; Mihajlovic et al., 2004] of drilling system and its modified version, supplemented by equations of induction motor, we demonstrate that unique stable equilibrium state can coexist with a stable limit cycle (hidden oscillation) in both models. It is very possible that the breakdowns in real drilling systems happen due to the existence of hidden oscillations in such systems, which are difficult to find because of the limitation of the standard numerical procedure.

\subsubsection{Two-mass mathematical model of drilling system}

The 'Two-mass' mathematical model of drilling system is a model, which allows one to perform indepth qualitative study of drill string behavior due to the fact that it can be described by a system of ordinary differential equations. Below, the model studied in [de Bruin et al., 2009; Mihajlovic et al., 2004] will be considered. This model consists of an upper disc, actuated by a drive part, no-mass string, and lower disc. The upper disc is connected to the lower disc by a string, which is a low stiffness connection between the discs. Here, there are two friction torques, acting on the upper and lower discs, respectively. The upper friction torque is caused by the electromagnetic field in the drive part of the model. The lower friction torque is caused by the friction against the workpiece which drill bit cuts. This model is described by equations of motion for the lower and upper discs:

$$
\begin{aligned}
& J_{u} \ddot{\theta}_{u}+k_{\theta}\left(\theta_{u}-\theta_{l}\right)+b\left(\dot{\theta}_{u}-\dot{\theta}_{l}\right) \\
& \quad+T_{f u}\left(\dot{\theta}_{u}\right)-k_{m} u=0, \\
& J_{l} \ddot{\theta}_{l}-k_{\theta}\left(\theta_{u}-\theta_{l}\right)-b\left(\dot{\theta}_{u}-\dot{\theta}_{l}\right)+T_{f l}\left(\dot{\theta}_{l}\right)=0 .
\end{aligned}
$$

Here $\theta_{u}$ and $\theta_{l}$ are angular displacements of the upper and lower discs respectively $\left(\dot{\theta}_{u}, \dot{\theta}_{l}\right.$ are derivatives of $\theta_{u}, \theta_{l}$ with respect to time $\left.t\right) ; J_{u}$ and $J_{l}$ are inertia torques; $k_{\theta}, k_{m}, b$ are non-negative coefficients, $u$ is a constant input voltage. Functions $T_{f u}$ and $T_{f l}$ are friction torques acting on the upper and lower discs (see Figs. 29 and 30):

$$
\begin{aligned}
& T_{f u}\left(\dot{\theta}_{u}\right) \in\left\{\begin{array}{r}
T_{c u}\left(\dot{\theta}_{u}\right) \operatorname{sign}\left(\dot{\theta}_{u}\right), \quad \text { for } \dot{\theta}_{u} \neq 0 \\
{\left[-T_{s u}+\Delta T_{s u}, T_{s u}+\Delta T_{s u}\right],} \\
\text { for } \dot{\theta}_{u}=0,
\end{array}\right. \\
& T_{c u}\left(\dot{\theta}_{u}\right)=T_{s u}+\Delta T_{s u} \operatorname{sign}\left(\dot{\theta}_{u}\right) \\
& +b_{u}\left|\dot{\theta}_{u}\right|+\Delta b_{u} \dot{\theta}_{u},
\end{aligned}
$$

where $T_{s u}, \Delta T_{s u}, b_{u}, \Delta b_{u}$ are non-negative coefficients.

$$
\begin{aligned}
& T_{f l}\left(\dot{\theta}_{l}\right) \in \begin{cases}T_{c l}\left(\dot{\theta}_{l}\right) \operatorname{sign}\left(\dot{\theta}_{l}\right), & \text { for } \dot{\theta}_{l} \neq 0 \\
{\left[-T_{s l}, T_{s l}\right],} & \text { for } \dot{\theta}_{l}=0,\end{cases} \\
& T_{c l}\left(\dot{\theta}_{l}\right)=T_{p}+\left(T_{s l}-T_{p}\right) e^{-\left|\frac{\dot{\theta}_{l}}{\omega_{s l}}\right| \delta_{s l}}+b_{l}\left|\dot{\theta}_{l}\right|,
\end{aligned}
$$




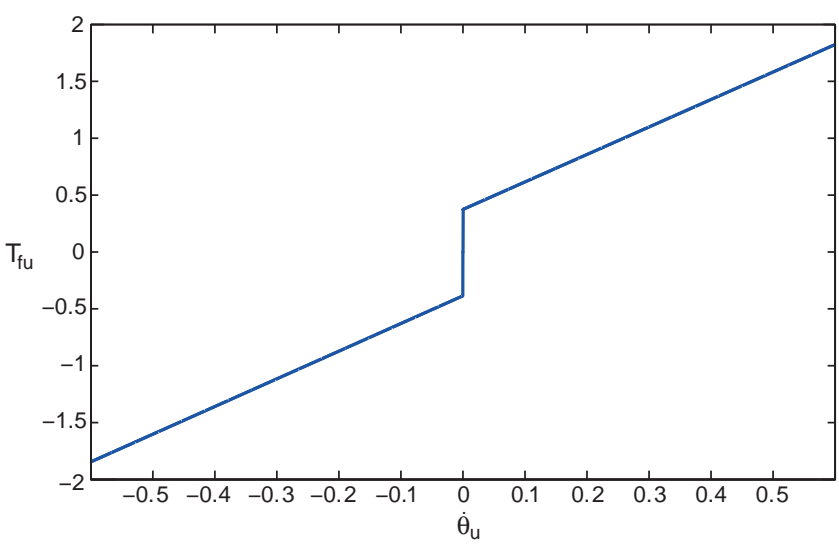

Fig. 29. Upper friction model $T_{f u}$.

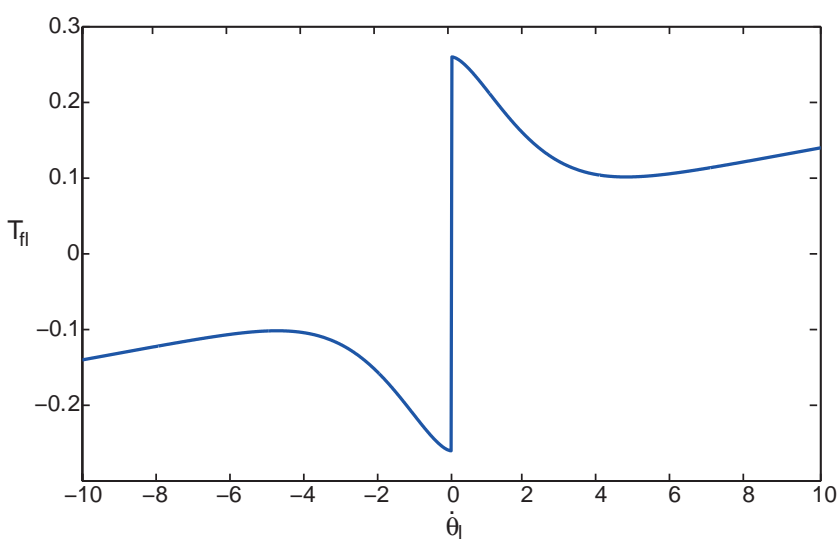

Fig. 30. Lower friction model $T_{f l}$.

where $T_{s l}, \quad T_{p}, \quad \omega_{s l}, \quad \delta_{s l}, \quad b_{l}$ are non-negative coefficients.

In this model, hidden oscillations can be found. Here, for $\omega_{u}=\dot{\theta}_{u}, \omega_{l}=\dot{\theta}_{l}, \alpha=\theta_{u}-\theta_{l}$, it can be seen that there is a stable equilibrium state $\alpha_{0} \approx 1.36$, $\omega_{u 0}=\omega_{l 0} \approx 5.17$ and a stable limit cycle (Fig. 31).

\subsubsection{Mathematical model of drilling system actuated by induction motor}

To take into account the dynamics of the rotor, a modified model of the drilling system actuated by the induction motor can be investigated. Consider the two-mass mathematical model of the drilling system (91) supplemented with equations of asynchronous motor:

$$
\begin{aligned}
& L \dot{i}_{1}+R i_{1}=\Phi_{B}\left(\sin \theta_{u}\right) \dot{\theta}_{u}, \\
& L \dot{i}_{2}+R i_{2}=\Phi_{B}\left(\cos \theta_{u}\right) \dot{\theta}_{u}, \\
& J_{u} \ddot{\theta}_{u}+k_{\theta}\left(\theta_{u}-\theta_{l}\right)+b\left(\dot{\theta}_{u}-\dot{\theta}_{l}\right) \\
& \quad+\beta \Phi_{B}\left(i_{1} \sin \theta_{u}+i_{2} \cos \theta_{u}\right)=0, \\
& \quad \ddot{\theta}_{l}-k_{\theta}\left(\theta_{u}-\theta_{l}\right)-b\left(\dot{\theta}_{u}-\dot{\theta}_{l}\right) \\
& \quad+T_{f l}\left(\omega+\dot{\theta}_{l}\right)=0 .
\end{aligned}
$$

Here $\theta_{u}, \theta_{l}$ are angular displacements of rotor and the lower disc relatively to the rotating magnetic field; $\omega$ is the speed of the rotation of magnetic field, $i_{1}, i_{2}$ are currents in the rotor windings; $T_{f l}\left(\omega+\dot{\theta}_{l}\right)$ is friction torque (same as in the first model); $R$ - resistance of windings; $L$ - inductance of windings; $\Phi_{B}$ - magnetic flux through the rotor; $\beta, J_{u}, J_{l}, k_{\theta}, b$ - non-negative coefficients. These equations are the modification of the first model. Here, the first two equations are the equations of the induction motor. In the third equation, the expression $T_{f u}\left(\dot{\theta}_{u}\right)-k_{m} u$ from the first model is replaced by the expression $\beta \Phi_{B}\left(i_{1} \sin \theta_{u}+i_{2} \cos \theta_{u}\right)$ which represents the effect of the induction motor on the upper disc. As was mentioned before, in contrast to the previous model here, $\theta_{u}$ and $\theta_{l}$ are

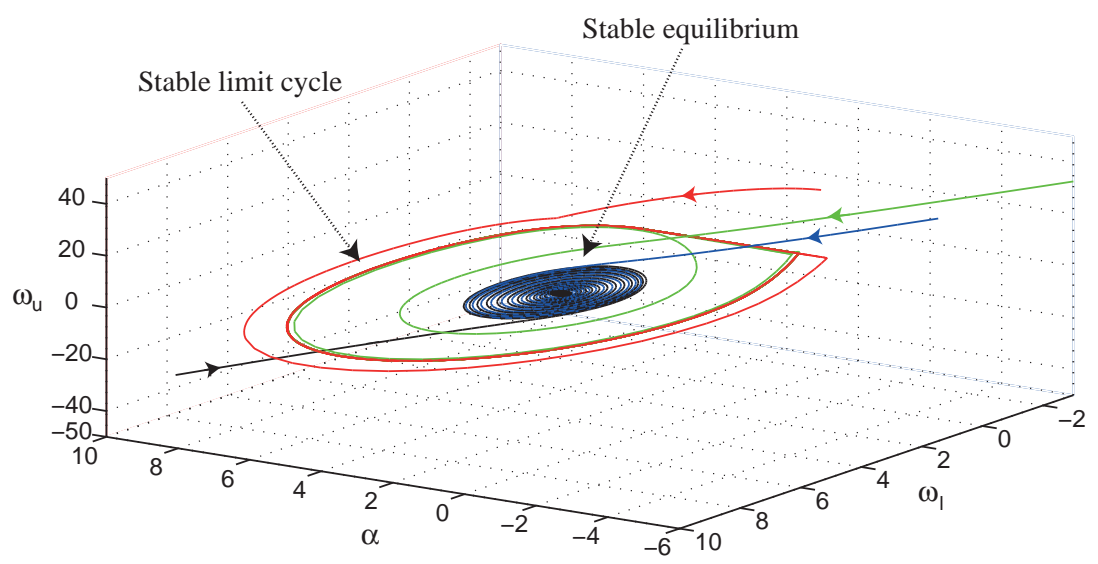

Fig. 31. Stable equilibrium and hidden oscillations — stable limit cycle. 


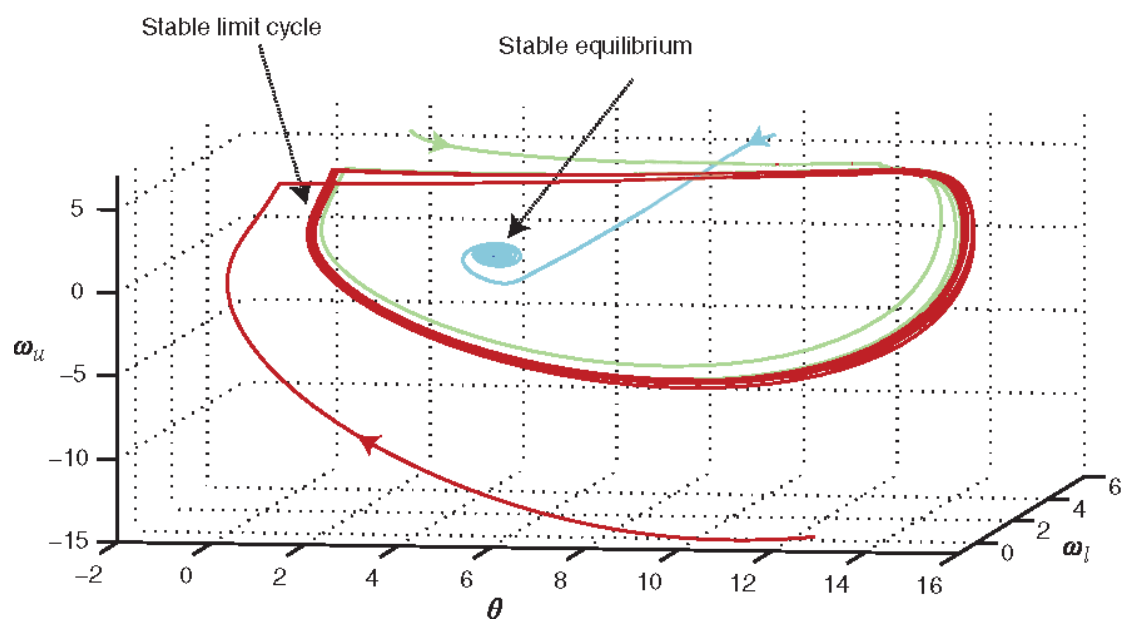

Fig. 32. Hidden oscillations and stable equilibrium in the mathematical model of drill actuated by induction motor.

angular displacements with respect to the rotating magnetic field. Only $T_{f l}(\dot{\theta})$ is changed to $T_{f l}\left(\omega+\dot{\theta}_{l}\right)$ due to the fact that $\left(\theta_{u}-\theta_{l}\right)$ is the same in both systems and, obviously, the derivatives of $\left(\omega+\dot{\theta}_{u}\right)$ and $\left(\omega+\dot{\theta}_{l}\right)$ are equal to $\dot{\theta}_{u}$ and $\dot{\theta}_{l}$, respectively.

The following change of variables

$$
\begin{aligned}
& \omega_{u}=-\dot{\theta}_{u}, \\
& x=\frac{L}{\Phi_{B}}\left(i_{1} \cos \theta_{u}-i_{2} \sin \theta_{u}\right), \\
& y=\frac{L}{\Phi_{B}}\left(i_{1} \sin \theta_{u}+i_{2} \cos \theta_{u}\right), \\
& \omega_{l}=-\dot{\theta}_{l}, \\
& \theta=\theta_{u}-\theta_{l},
\end{aligned}
$$

results in the system of fifth order

$$
\begin{aligned}
& \dot{y}=-c y-\omega_{u}-x \omega_{u}, \\
& \dot{x}=-c x+y \omega_{u}, \\
& \dot{\theta}=\omega_{l}-\omega_{u}, \\
& \dot{\omega}_{u}=\frac{k_{\theta}}{J_{u}} \theta+\frac{b}{J_{u}}\left(\omega_{l}-\omega_{u}\right)+\frac{a}{J_{u}} y, \\
& \dot{\omega}_{l}=-\frac{k_{\theta}}{J_{l}}-\frac{b}{J_{l}}\left(\omega_{l}-\omega_{u}\right)+\frac{1}{J_{l}} T_{f l}\left(\omega-\omega_{l}\right) .
\end{aligned}
$$

Here $a=\frac{\beta \Phi_{B}{ }^{2}}{L}, c=\frac{R}{L}$.

By computer simulation, it is shown that under certain parameters the system has stable equilibrium state and hidden oscillation stable limit cycle (see Fig. 32).

\section{Aizerman's and Kalman's Conjectures on Absolute Stability of Control System}

In 1949, Aizerman formulated the problem [Aizerman, 1949], which captured the attention of many famous scholars, working in the field of control theory and differential equations (see, e.g. [Aizerman \& Gantmacher, 1964; Lefschetz, 1965]). The Aizerman's conjecture is formulated in the following way. Consider a system with one scalar nonlinearity

$$
\frac{d \mathbf{x}}{d t}=\mathbf{P} \mathbf{x}+\mathbf{q} \psi\left(\mathbf{r}^{*} \mathbf{x}\right), \quad \mathbf{x} \in \mathbb{R}^{n},
$$

where $\mathbf{P}$ is a constant $n \times n$-matrix, $\mathbf{q}, \mathbf{r}$ are constant $n$-dimensional vectors, ${ }^{*}$ is an operation of transposition, $\psi(\sigma)$ is a piecewise-continuous scalar function, and $\psi(0)=0$. Here the solutions of system (98) are interpreted in the sense of Filippov [1988]. Suppose that all linear systems (98) with

$$
\psi(\sigma)=\mu \sigma, \quad \mu \in\left(\mu_{1}, \mu_{2}\right)
$$

are asymptotically stable. The question arises whether system (98) with any nonlinearity $\psi(\sigma)$, under the condition

$$
\mu_{1}<\frac{\psi(\sigma)}{\sigma}<\mu_{2}, \quad \forall \sigma \neq 0,
$$

is stable in the large (i.e. a zero solution of system (98) is asymptotically stable and any solution tends to zero as $t \rightarrow+\infty)$.

In 1952, Aizerman's conjecture was completely proved for $n=2$ due to Malkin [1952], Erugin [1952], and Krasovsky [1952]. In this case, for 
$n=2$, Aizerman's conjecture always has a positive solution except for the case when the matrix $(\mathbf{P}+$ $\left.\mu_{1} \mathbf{q r}^{*}\right)$ has a double zero eigenvalue and

$$
\begin{aligned}
& \int_{0}^{\infty}\left(\psi(\sigma)-\mu_{1} \sigma\right) d \sigma \neq+\infty \text { or } \\
& \int_{0}^{-\infty}\left(\psi(\sigma)-\mu_{1} \sigma\right) d \sigma \neq-\infty .
\end{aligned}
$$

Krasovsky [1952] showed that in the case when these conditions are satisfied, system (98) can have solutions tending to infinity. This was the first counterexample to Aizerman's conjecture, which was generalized further to systems (98) of arbitrary order [Pliss, 1958; Yakubovich, 1958; Efendiev \& Balitinov, 1968; Leonov, 1981].

In 1957, Kalman [1957] stated the following: "If $f(e)$ in Fig. 1 [see Fig. 33] is replaced by constants $K$ corresponding to all possible values of $f^{\prime}(e)$, and it is found that the closed-loop system is stable for all such $K$, then it is intuitively clear that the system must be monostable; i.e. all transient solutions will converge to a unique, stable critical point."

Kalman's statement can be reformulated in the following conjecture (Kalman's conjecture) [Bragin et al., 2011; Leonov \& Kuznetsov, 2013a]: Suppose, $\psi(\sigma)$ is a piecewise-differentiable function ${ }^{1}$ and at the points of differentiability the following condition

$$
\mu_{1}<\psi^{\prime}(\sigma)<\mu_{2}
$$

is valid. The question arises whether system (98) is stable in the large if all linear systems (98) with $\psi(\sigma)=\mu \sigma, \mu \in\left(\mu_{1}, \mu_{2}\right)$ are asymptotically stable.

Note that if Kalman's conjecture conditions are satisfied, then Aizerman's conjecture conditions are also satisfied. It is clear that for $n=2$, the case, considered by Krasovsky in his counterexample, is not possible under Kalman's conditions, therefore,

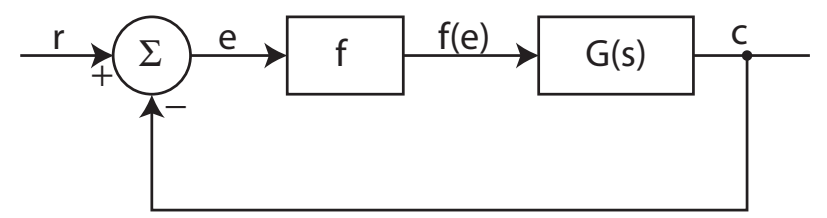

Fig. 33. Nonlinear control system. $G(s)$ is a linear transfer function, $f(e)$ is a single-valued, continuous, and differentiable function [Kalman, 1957]. for $n=2$, Kalman's problem has a positive solution. In 1958, Pliss [1958] developed a method for the construction of three-dimensional nonlinear systems, satisfying the condition of Aizerman's conjecture and having periodic solutions. Then this method was extended to systems (98) of arbitrary dimension [Leonov, 1970; Noldus, 1971]. However, as before, the classes of these systems did not satisfy Kalman's condition. Later, it was shown that the frequency criteria of stability imply a positive solution of Kalman's problem for $n=2$ and $n=3$ [Leonov et al., 1996].

The extension of the question, formulated in Kalman's problem, to the case of multidimensional nonlinearity is known as Markus-Yamabe conjecture [Markus \& Yamabe, 1960].

Suppose, for a system

$$
\dot{\mathbf{x}}=\mathbf{f}(\mathbf{x}), \quad \mathbf{f}: \mathbb{R}^{n} \rightarrow \mathbb{R}^{n}, \quad \mathbf{f} \in \mathcal{C}^{1}, \quad \mathbf{f}(\mathbf{0})=\mathbf{0}
$$

the Jacobian matrix $\left(\frac{d \mathbf{f}(\mathbf{x})}{d \mathbf{x}}\right)$ has all eigenvalues with negative real parts for all $\mathbf{x} \in \mathbb{R}^{n}$. The question arises whether system (100) is stable in the large.

This problem has a positive solution for $n=2$ [Glutsyuk, 1995; Feßler, 1995; Gutierrez, 1995] and a negative solution in the general case for $n \geq 3$. For example, a polynomial system, considered in [Cima et al., 1997],

$$
\begin{aligned}
& \dot{x}_{1}=-x_{1}+x_{3}\left(x_{1}+x_{2} x_{3}\right)^{2}, \\
& \dot{x}_{2}=-x_{2}-\left(x_{1}+x_{2} x_{3}\right)^{2}, \\
& \dot{x}_{3}=-x_{3}
\end{aligned}
$$

has the Jacobian matrix with three eigenvalues equal to -1 , but at the same time, it allows the unbounded solution $\left(x_{1}(t), x_{2}(t), x_{3}(t)\right)=$ $\left(18 e^{t},-12 e^{2 t}, e^{-t}\right)$.

Some recent development on the existence of periodic solution and absolute stability theory, related to Aizerman and Kalman conjectures, is presented, e.g. in [Rasvan, 2004; Margaliota \& Yfoulis, 2006; Llibre et al., 2011; Grabowski, 2011; Alli-Oke et al., 2012].

Within the framework of investigation of the above conjectures and unlike Krasovsky and Cima counterexamples, from the applied point of view, [Kalman, 1957]: "Undoubtedly the most important instability of interest in control systems are limit cycles; the circumstances which lead to creation or

\footnotetext{
${ }^{1}$ i.e. a function, having finite number of points of discontinuity on any interval, and differentiated on closed continuity intervals.
} 
destruction of limit cycles are to be studied in considerable detail. Solutions tending to infinity are also fairly readily avoidable...".

Later in studying applied systems, hidden chaotic attractors [Kuznetsov et al., 2010; Leonov et al., 2010c; Bragin et al., 2011; Leonov et al., 2011c; Leonov \& Kuznetsov, 2011b; Leonov et al., 2011b, 2012a; Kuznetsov et al., 2013; Leonov \& Kuznetsov, 2013a, 2013b] were also discovered, further the efforts will be focused on the construction of effective methods for the analysis of hidden periodic oscillations and chaotic attractors.

\subsection{Analytical-numerical procedure for hidden attractors localization}

It was found that the effective methods for the numerical localization of hidden attractors in multidimensional dynamical systems are the methods based on numerical continuation: a sequence of similar systems is constructed such that for the first (starting) system the initial data for numerical computation of possible oscillating solution (starting oscillation) can be obtained analytically and then the transformation of this starting oscillation when passing from one system to another is followed numerically.

In a scenario of transition to chaos in dynamical system, there is usually a parameter $\lambda \in[a, b]$, its varying gives this scenario. We introduce the parameter $\lambda$ artificially, and then consider its varying on the interval $[a, b]$ such that $\lambda=b$ corresponds to the initial system, and the parameter $a$ is chosen in such a way that for $\lambda=a$ it is possible to compute or localize a certain attractor (often such an attractor is of simple form). Further, a sequence $\lambda_{j}, \lambda_{1}=a, \lambda_{n}=b, \lambda_{j} \in[a, b]$ is considered, such that the distance between $\lambda_{j}$ and $\lambda_{j+1}$ is sufficiently small. Then the change of the structure of an attractor, obtained for $\lambda_{1}=a$, is considered numerically. If during the change of the parameter $\lambda$ (from $\lambda_{j}$ to $\lambda_{j+1}$ ) there is no loss of stability bifurcation of the considered attractor, then for $\lambda_{n}=b$ at the end of the procedure, an attractor (often hidden or difficult to detect) of the initial system is localized. Emphasize again that in this case, the scenario is usually organized artificially. In other words, here instead of the analysis of scenario of transition to chaos, a scenario of transition to chaos is synthesized.
Further, an example of effective analyticalnumerical approach, for hidden oscillations localization, which is based on the method of small parameter, the harmonic linearization and describing function method, numerical methods, and applied bifurcation theory, is discussed, following mainly the works [Leonov, 2009c, 2009d, 2009b, 2010a].

Define a coefficient of harmonic linearization $k$ (suppose that such $k$ exists) in such a way that the matrix

$$
\mathbf{P}_{0}=\mathbf{P}+k \mathbf{q} \mathbf{r}^{*}
$$

has a pair of purely imaginary eigenvalues $\pm i \omega_{0}$ $\left(\omega_{0}>0\right)$ and the rest of its eigenvalues has negative real parts. Rewrite system (98) as

$$
\frac{d \mathbf{x}}{d t}=\mathbf{P}_{\mathbf{0}} \mathbf{x}+\mathbf{q} \varphi\left(\mathbf{r}^{*} \mathbf{x}\right)
$$

where $\varphi(\sigma)=\psi(\sigma)-k \sigma$.

Introduce a finite sequence of functions $\varphi^{0}(\sigma)$, $\varphi^{1}(\sigma), \ldots, \varphi^{m}(\sigma)$ such that the graphs of neighboring functions $\varphi^{j}(\sigma)$ and $\varphi^{j+1}(\sigma)$ slightly differ from one another, the function $\varphi^{0}(\sigma)$ is small, and $\varphi^{m}(\sigma)=\varphi(\sigma)$. By a smallness of the function $\varphi^{0}(\sigma)$, for the system

$$
\frac{d \mathbf{x}}{d t}=\mathbf{P}_{\mathbf{0}} \mathbf{x}+\mathbf{q} \varphi^{0}\left(\mathbf{r}^{*} \mathbf{x}\right)
$$

the describing function method can be used and be mathematically strictly justified [Leonov, 2010a; Leonov et al., 2010c, 2010a]. Its application allows to define a stable nontrivial periodic solution $\mathbf{x}^{0}(t)$. Then for the localization of an attractor of original system (102), we can follow numerically the transformation of this periodic solution $\mathbf{x}^{0}(t)$ (a starting oscillating attractor is an attractor, not including equilibria and denoted further by $\mathcal{A}_{0}$ ) with increasing $j$.

Here two cases are possible. The first case: all the points of $\mathcal{A}_{0}$ are in an attraction domain of the attractor $\mathcal{A}_{1}$, which is an oscillating attractor of the system

$$
\frac{d \mathbf{x}}{d t}=\mathbf{P}_{0} \mathbf{x}+\mathbf{q} \varphi^{j}\left(\mathbf{r}^{*} \mathbf{x}\right)
$$

with $j=1$. The second case: when passing from system (103) to system (104) with $j=1$, a loss of stability is observed and the vanishing of $\mathcal{A}_{0}$. In the first case, the solution $\mathbf{x}^{1}(t)$ can be defined numerically by starting a trajectory of system (104) with $j=1$ from the initial point $\mathbf{x}^{0}(0)$. If in the process of computation (here it should be considered a sufficiently large computational interval $[0, T]$ ) the 
solution $\mathbf{x}^{1}(t)$ does not fall to an equilibrium and is not increased indefinitely, then this solution reaches an attractor $\mathcal{A}_{1}$. In this case, it is possible to proceed to system (104) with $j=2$ and to perform a similar procedure of computation of $\mathcal{A}_{2}$ by starting from a trajectory of system (104) with $j=2$ from the initial point $\mathbf{x}^{1}(T)$ and computing a trajectory $\mathbf{x}^{2}(t)$.

Proceeding further, sequentially increasing $j$, and computing $\mathbf{x}^{j}(t)$ (a trajectory of system (104) with the initial data $\mathrm{x}^{j-1}(T)$ ), one can either compute $\mathcal{A}_{m}$ (an attractor of system (104) with $j=m$, i.e. original system (102)), or observe, at a certain step, a loss of stability and the vanishing of attractor.

Here the form of the linear part of the systems and the smallness of the function $\varphi^{0}(\sigma)$ permit one to strictly determine the starting periodic solution by the describing function method.

Note that in many comparatively simple cases, more simple algorithms can be used for the construction of the corresponding sequence of systems, based on varying one of the system coefficients in such a way that a starting system has a self-excited oscillation. This starting self-excited oscillation can be obtained by the standard computational procedure, and then a sequential transformation of the starting self-excited oscillation to hidden oscillation can be followed numerically. Different realizations of numerical continuation methods are discussed, e.g. in [Doedel, 1986; Allgower \& Georg, 1990; Beyn et al., 2002; Krauskopf et al., 2007].

\subsection{Small parameter and describing function method}

The method of harmonic linearization (harmonic balance, describing function method - DFM) is widely known and is often applied to the analysis of oscillations in nonlinear systems of automatic control (see, e.g. some recent developments and applications [Tesi et al., 1996; Collera \& Chamara, 2004; Aracil \& Gordillo, 2004; Zhou \& Zhang, 2005; Liu et al., 2006; LaBryer \& Attar, 2010; Chen et al., 2012]). This method is not strictly mathematically justified and belongs to approximate methods of analysis of control systems (see, e.g. [Krylov \& Bogolyubov, 1947; Khalil, 2002]). One of the first examples, where the describing function method gives untrue results, is due to Tzipkin [1984].
Below, we will describe the application of describing function method to Aizerman's conjecture, which leads to wrong conclusion. For this purpose, recall a standard way of applying the describing function method to system (98). This procedure is the following. Introduce a transfer function

$$
W(p)=\mathbf{r}^{*}(\mathbf{P}-p \mathbf{I})^{-1} \mathbf{q},
$$

where $p$ is a complex variable, $\mathbf{I}$ is a unit matrix. Let us find a harmonic oscillation $a \cos \omega_{0} t$, which is an approximate solution of system (98):

$$
\sigma(t)=\mathbf{r}^{*} \mathbf{x}(t) \approx a \cos \omega_{0} t
$$

Define first the coefficient of harmonic linearization $k$ and the frequency $\omega_{0}$. For solving practical problems of defining the values of $k$ and $\omega_{0}$, a transfer function $W(p)$ is applied. The number $\omega_{0}$ is defined from the equation

$$
\operatorname{Im} W\left(i \omega_{0}\right)=0
$$

and $k$ is defined by the formula

$$
k=-\left(\operatorname{Re} W\left(i \omega_{0}\right)\right)^{-1} .
$$

Following the describing function method, the amplitude $a$ can be obtained from the equation

$$
\begin{array}{r}
\int_{0}^{2 \pi / \omega_{0}} \psi\left(a \cos \omega_{0} t\right) \cos \omega_{0} t d t \\
=k a \int_{0}^{2 \pi / \omega_{0}}\left(\cos \omega_{0} t\right)^{2} d t .
\end{array}
$$

Now the procedure described is applied to Aizerman's conjecture. It is clear that in the considered case, the condition $k \in\left(\mu_{1}, \mu_{2}\right)$ (see $\left.(99)\right)$ is not satisfied. Then for any nonzero value $\sigma$, one of the estimates

$$
k \sigma^{2}<\psi(\sigma) \sigma \quad \text { or } \quad \psi(\sigma) \sigma<k \sigma^{2}
$$

is valid. This implies that for all $a \neq 0$ the inequality

$$
\begin{gathered}
\int_{0}^{2 \pi / \omega_{0}}\left(\psi\left(a \cos \omega_{0} t\right) a \cos \omega_{0} t\right. \\
\left.-k\left(a \cos \omega_{0} t\right)^{2}\right) d t \neq 0
\end{gathered}
$$

is satisfied. Thus, according to the describing function method, system (98) under the conditions of Aizerman's conjecture (and also Kalman's conjecture) has no periodic solutions. However the results of Pliss and its progeny [Leonov, 1970; Noldus, 1971] are in contrast to these conclusions which were obtained by the describing function method. 
This is why, for many decades, attempts were made to find classes of systems for which the describing function method (and its various extensions) gives true results. The first such works were due to Bulgakov [1943, 1954], where a variant of the classical method of small parameter was used.

Later, this direction falls under serious critique, which is reduced to the following "these methods of small parameter are based on the assumption that the original small system differs from the linear system, having self-generating frequency. In the theory of automatic control, similar assumptions cannot be made since the system, as is known, is not conservative and in linear approximation stability conditions are satisfied with sufficient fund" (translated into English from [Aizerman, 1953]). With provision for this criticism, other methods of entering the small parameter, based on the filter hypothesis, were developed [Garber \& Rozenvasser, 1965; Bergen \& Franks, 1971; Mees \& Bergen, 1975].

The development of numerical methods, computers and applied theory of bifurcations permit one to return to the first ideas, related to the application of the methods of small parameter and the describing function method in dynamical systems, and to consider them with new standpoints.

Here, the method of harmonic linearization will be modified and justified. This will be done in such a way that the method can be regarded as a base for the construction of algorithms in the search of oscillations in Aizerman's and Kalman's conjectures.

\subsection{Describing function method justification}

\subsubsection{System reduction}

To define the initial data $\mathbf{x}^{0}(0)$ of starting periodic solution, system (103) with the nonlinearity $\varphi^{0}(\sigma)$ is transformed by a linear nonsingular transformation $\mathbf{x}=\mathbf{S y}$ to the form

$$
\begin{aligned}
& \dot{y}_{1}=-\omega_{0} y_{2}+b_{1} \varphi^{0}\left(y_{1}+\mathbf{c}_{3}^{*} \mathbf{y}_{3}\right), \\
& \dot{y}_{2}=\omega_{0} y_{1}+b_{2} \varphi^{0}\left(y_{1}+\mathbf{c}_{3}^{*} \mathbf{y}_{3}\right), \\
& \dot{\mathbf{y}}_{3}=\mathbf{A}_{3} \mathbf{y}_{3}+\mathbf{b}_{3} \varphi^{0}\left(y_{1}+\mathbf{c}_{3}^{*} \mathbf{y}_{3}\right) .
\end{aligned}
$$

Here $y_{1}, y_{2}$ are scalars, $\mathbf{y}_{3}$ is $(n-2)$-dimensional vector; $\mathbf{b}_{3}$ and $\mathbf{c}_{3}$ are $(n-2)$-dimensional vectors, $b_{1}$ and $b_{2}$ are real numbers; $\mathbf{A}_{3}$ is an $((n-2) \times(n-2))$ matrix, all eigenvalues of which have negative real parts. Without loss of generality, it can be assumed that for the matrix $\mathbf{A}_{3}$ there exists a positive number $d>0$ such that

$$
\mathbf{y}_{3}^{*}\left(\mathbf{A}_{3}+\mathbf{A}_{3}^{*}\right) \mathbf{y}_{3} \leq-2 d\left|\mathbf{y}_{3}\right|^{2}, \quad \forall \mathbf{y}_{3} \in \mathbb{R}^{n-2} .
$$

Let us present a transfer function of system (103)

$$
\mathbf{r}^{*}\left(\mathbf{P}_{\mathbf{0}}-p \mathbf{I}\right)^{-1} \mathbf{q}=\frac{\eta p+\theta}{p^{2}+\omega_{0}^{2}}+\frac{R(p)}{Q(p)}
$$

and a transfer function of system (106)

$$
\frac{-b_{1} p+b_{2} \omega_{0}}{p^{2}+\omega_{0}^{2}}+\mathbf{c}_{3}{ }^{*}\left(\mathbf{A}_{3}-p \mathbf{I}\right)^{-1} \mathbf{b}_{3} .
$$

Here $\eta$ and $\theta$ are certain real numbers, $Q(p)$ is a stable polynomial of degree $(n-2), R(p)$ is a polynomial of degree smaller than $(n-2)$. Suppose, the polynomials $R(p)$ and $Q(p)$ have no common roots. From the equivalence of systems (103) and (106), it follows that the transfer functions of these systems coincide. This implies the following relations

$$
\begin{aligned}
& \eta=-b_{1}, \quad \theta=b_{2} \omega_{0}, \quad \mathbf{c}_{\mathbf{3}}{ }^{*} \mathbf{b}_{3}+b_{1}=\mathbf{r}^{*} \mathbf{q} \\
& \frac{R(p)}{Q(p)}=\mathbf{c}_{\mathbf{3}}{ }^{*}\left(\mathbf{A}_{3}-p \mathbf{I}\right)^{-1} \mathbf{b}_{3} .
\end{aligned}
$$

\subsubsection{Poincaré map for harmonic linearization in the noncritical case}

At the first step of algorithm, we consider system (106) with the nonlinearity $\varphi^{0}(\sigma)=\varepsilon \varphi(\sigma)(\varepsilon$ is an artificial continuation parameter)

$$
\begin{aligned}
& \dot{y}_{1}=-\omega_{0} y_{2}+b_{1} \varepsilon \varphi\left(y_{1}+\mathbf{c}_{\mathbf{3}}{ }^{*} \mathbf{y}_{3}\right), \\
& \dot{y}_{2}=\omega_{0} y_{1}+b_{2} \varepsilon \varphi\left(y_{1}+\mathbf{c}_{\mathbf{3}}{ }^{*} \mathbf{y}_{3}\right), \\
& \dot{\mathbf{y}}_{3}=\mathbf{A}_{3} \mathbf{y}_{3}+\mathbf{b}_{3} \varepsilon \varphi\left(y_{1}+\mathbf{c}_{3}{ }^{*} \mathbf{y}_{3}\right),
\end{aligned}
$$

where $\varphi(\sigma)$ is a piecewise-differentiable function with discontinuity points $\nu_{i}$.

Consider in phase space of nonlinear system (111) the following set

$$
\Omega=\left\{y_{1} \in\left[a_{1}, a_{2}\right], y_{2}=0,\left|\mathbf{y}_{3}\right| \leq D \varepsilon\right\},
$$

where $a_{1,2}$ are certain positive numbers. The number $D$ is defined according to the following assertion.

Lemma 8 [Leonov, 2010a]. The numbers $D_{1} \geq D>$ 0 can be found such that if for sufficiently small 
$\varepsilon>0$ the inequality

$$
\left|\mathbf{y}_{3}(0)\right| \leq D \varepsilon
$$

is satisfied, then

$$
\left|\mathbf{y}_{3}(T)\right| \leq D \varepsilon
$$

and

$$
\left|\mathbf{y}_{3}(t)\right| \leq D_{1} \varepsilon, \quad \forall t \in[0, T] .
$$

Here $T$ is return time of trajectories of system (111) in Poincaré map $F$ of the set $\Omega$ :

$$
F\left\|\begin{array}{c}
y_{1}(0) \\
0 \\
\mathbf{y}_{3}(0)
\end{array}\right\|=\left\|\begin{array}{c}
y_{1}(T) \\
0 \\
\mathbf{y}_{3}(T)
\end{array}\right\| .
$$

Thus $T$ is a positive number for which

$$
y_{1}(T)>0, \quad y_{2}(T)=0,
$$

and the relations

$$
y_{1}(t)>0, \quad y_{2}(t)=0, \quad \forall t \in(0, T)
$$

are not satisfied.

Introduce the following describing function

$$
\Phi(a)=\int_{0}^{2 \pi / \omega_{0}} \varphi\left(\cos \left(\omega_{0} t\right) a\right) \cos \left(\omega_{0} t\right) d t
$$

Theorem 9 [Leonov, 2010a]. If $a_{1,2} \neq\left|\nu_{i}\right|$ and the inequalities

$$
b_{1} \Phi\left(a_{1}\right)>0, \quad b_{1} \Phi\left(a_{2}\right)<0
$$

are satisfied, then for sufficiently small $\varepsilon>0$, Poincaré map (114) of the set $\Omega$ is mapped into itself: $F \Omega \subset \Omega$.

This theorem and Brouwer's theorem on fixed point imply the following

Corollary 4.1. If $a_{1,2} \neq\left|\nu_{i}\right|$ and inequalities (116) are satisfied, then for sufficiently small $\varepsilon>0$ system (111) has a periodic solution with initial data from $\Omega$. This solution is stable in the sense that its neighborhood $\Omega$ is mapped into itself $: F \Omega \subset \Omega$.

The existence of derivative of the describing function results in the following

Theorem 10. Suppose that there exists a number $a_{0}>0, a_{0} \neq\left|\nu_{i}\right|$ such that the conditions

$$
\Phi\left(a_{0}\right)=0,\left.\quad b_{1} \frac{d \Phi(a)}{d a}\right|_{a=a_{0}}<0
$$

are satisfied. Then for sufficiently small $\varepsilon>0$ system (111) has a periodic solution of the form

$$
\begin{aligned}
& y_{1}(t)=\cos \left(\omega_{0} t\right) y_{1}(0)+O(\varepsilon), \\
& y_{2}(t)=\sin \left(\omega_{0} t\right) y_{1}(0)+O(\varepsilon), \quad t \in[0, T] \\
& \mathbf{y}_{3}(t)=\exp \left(\mathbf{A}_{3} t\right) \mathbf{y}_{3}(0)+\mathbf{O}_{\mathbf{n}-\mathbf{2}}(\varepsilon),
\end{aligned}
$$

with the initial data

$$
\begin{aligned}
& y_{1}(0)=a_{0}+O(\varepsilon), \\
& y_{2}(0)=0, \\
& \mathbf{y}_{3}(0)=\mathbf{O}_{\mathbf{n}-\mathbf{2}}(\varepsilon)
\end{aligned}
$$

and with the period

$$
T=\frac{2 \pi}{\omega_{0}}+O(\varepsilon) .
$$

Here $\mathbf{O}_{\mathbf{n}-\mathbf{2}}(\varepsilon)$ is an $(n-2)$-dimensional vector such that its components are $O(\varepsilon)$.

Taking into account relations (110), this theorem can be reformulated

Corollary 4.2. Suppose that there exists a number $a_{0}>0, a_{0} \neq\left|\nu_{i}\right|$ such that the conditions

$$
\Phi\left(a_{0}\right)=0,\left.\quad \eta \frac{d \Phi(a)}{d a}\right|_{a=a_{0}}>0
$$

are satisfied. Then for sufficiently small $\varepsilon>0$ system (103) with transfer function (108) and the nonlinearity $\varphi^{0}(\sigma)=\varepsilon \varphi(\sigma)$ has a T-periodic solution such that

$$
\mathbf{r}^{*} \mathbf{x}(t)=a_{0} \cos \left(\omega_{0} t\right)+O(\varepsilon), \quad T=\frac{2 \pi}{\omega_{0}}+O(\varepsilon) .
$$

Theorem 10 describes the procedure of the search for stable periodic solutions by the standard describing function method (see, e.g. [Khalil, 2002]).

Similar assertions can also be formulated in the case of vector nonlinearity [Kuznetsov et al., 2010; Leonov et al., 2010c].

A sketch of proof of Theorem 9. The proof of this theorem is due to the following lemmas.

Lemma 9. In the case of sufficiently small $\varepsilon$ on a finite time interval for the solution of system (111) 
with initial data from $\Omega$, representation (118) occurs.

Lemma 10. For $y_{1}(0) \neq\left|\nu_{i}\right|$ the following estimate

$$
\begin{aligned}
\int_{0}^{2 \pi / \omega_{0}} \varphi(\sigma(t)) d t \\
\quad=\int_{0}^{2 \pi / \omega_{0}} \varphi\left(\cos \left(\omega_{0} t\right) y_{1}(0)\right) d t+O(\varepsilon)
\end{aligned}
$$

is satisfied.

Lemma 11. Suppose, $y_{1}(0) \neq\left|\nu_{i}\right|$. Then for sufficiently small $\varepsilon>0$ the estimate

$$
\begin{aligned}
& y_{1}(T)^{2}-y_{1}(0)^{2} \\
& \quad=2 \varepsilon y_{1}(0) b_{1} \Phi\left(y_{1}(0)\right)+O\left(\varepsilon^{2}\right)
\end{aligned}
$$

is satisfied.

For the proof of this lemma one can use the following Lyapunov function: $V\left(y_{1}, y_{2}\right)=y_{1}^{2}+y_{2}^{2}$.

These lemmas imply that the map $F$ of the set $\Omega$ is mapped into itself.

It should be noted that, as was shown above, condition (117) cannot be satisfied in the critical case when the conditions of Aizerman's and Kalman's conjectures are fulfilled (i.e. a nonlinearity belongs to the sector of linear stability). In this critical case, the harmonic balance and describing function method lead to a wrong result - to the nonexistence of periodic solutions and global stability of unique stationary point, but nowadays the counterexamples are well known (see, e.g. [Leonov et al., 2010a, 2010b; Kuznetsov et al., 2011b; Bragin et al., 2011; Leonov \& Kuznetsov, 2011a, 2011b; Leonov et al., 2011b]).

Now consider some examples of applying the given algorithm.

Example 1. Suppose, $\varphi(\sigma)=\sigma-\operatorname{sign} \sigma$. Then

$$
\Phi(a)=\frac{\pi a-4}{\omega_{0}} .
$$

This implies that $a$ is defined from the equation $\Phi(a)=0$ in the following way:

$$
a=a_{0}=\frac{4}{\pi}
$$

and the condition of stability takes the form $\eta>0$.
Consider now system (102) with transfer function

$$
W(p)=\frac{p+1}{p^{2}+1}-\frac{1}{p+1}
$$

Then

$$
\begin{aligned}
& \omega_{0}=1, \quad b_{1}=-1, \quad b_{2}=1, \\
& \mathbf{A}_{3}=-1, \quad \mathbf{c}_{3}=1, \quad \mathbf{b}_{3}=1 .
\end{aligned}
$$

Using a classical describing function method, one can obtain that for any $\varepsilon>0$ system (103) (or (111)) has a periodic solution and

$$
\sigma(t)=\mathbf{r}^{*} \mathbf{x}(t) \approx a_{0} \cos t .
$$

By Theorem 9 for small $\varepsilon>0$, system (111) has the stable periodic solution of the form

$$
\begin{aligned}
& x_{1}(t)=a_{0} \cos t+O(\varepsilon), \\
& x_{2}(t)=a_{0} \sin t+O(\varepsilon), \\
& x_{3}(t)=O(\varepsilon) .
\end{aligned}
$$

Consider now the transformation of thus solution with increasing $\varepsilon$ by discrete steps from 0.1 to 1 . Computational procedures are constructed as was described above.

In Fig. 34 are shown the projections of the computed periodic solutions on the plane $\left\{x_{1}, x_{2}\right\}$. Note that the output $\sigma(t)=x_{1}(t)+x_{3}(t)$ is close to harmonic and here the filter hypothesis [Khalil, 2002] is valid. Thus, in this case it is possible, in principle, to justify the standard describing function method for the considered values $\varepsilon$.

Example 2. Suppose that $\varphi(\sigma)=k_{1} \sigma+k_{3} \sigma^{3}$. Then

$$
\Phi(a)=\left(k_{1} a+\frac{3}{4} k_{3} a^{3}\right) \frac{\pi}{\omega_{0}} .
$$

This implies that $a$ can be obtained from the equation $\Phi(a)=0$ :

$$
a=a_{1}=\sqrt{-\frac{4 k_{1}}{3 k_{3}}},
$$

and a stability condition takes the form $\eta k_{1}<0$.

Consider once more system (102) with $W(p)$ of the form (123). Suppose, $k_{1}=-3, k_{3}=4$. Then $a_{1}=1$.

By standard DFM, it can be obtained that for any $\varepsilon>0$, system (103) (or (111)) has a periodic 

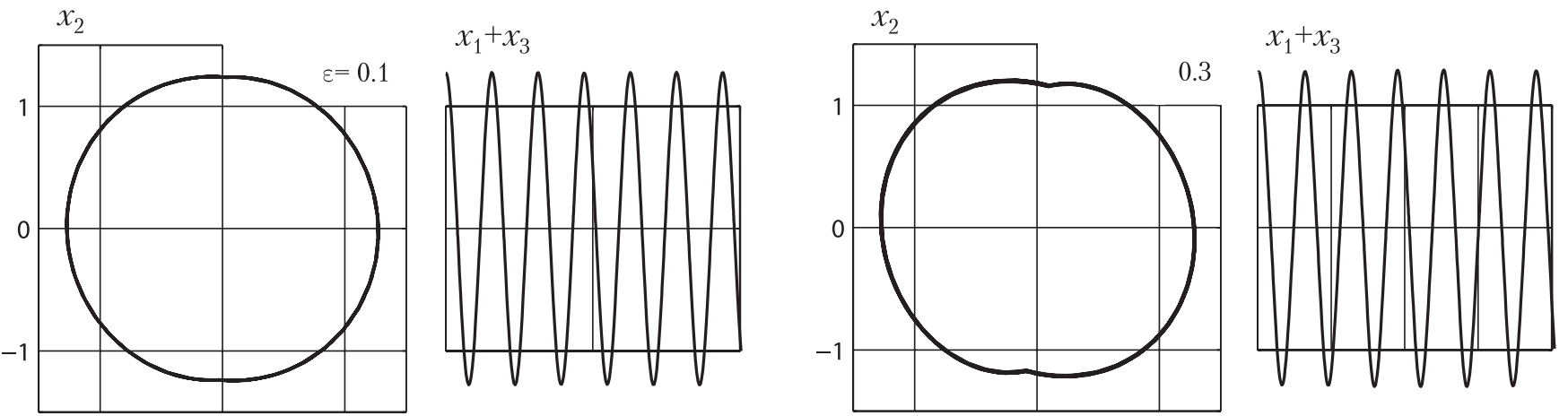
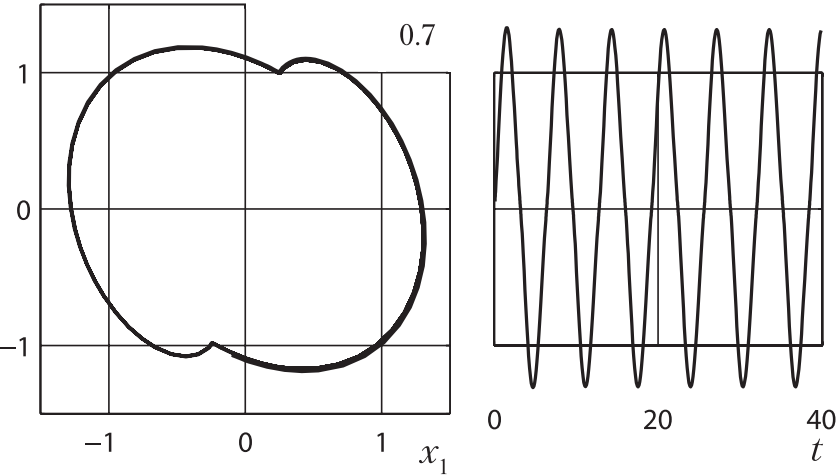
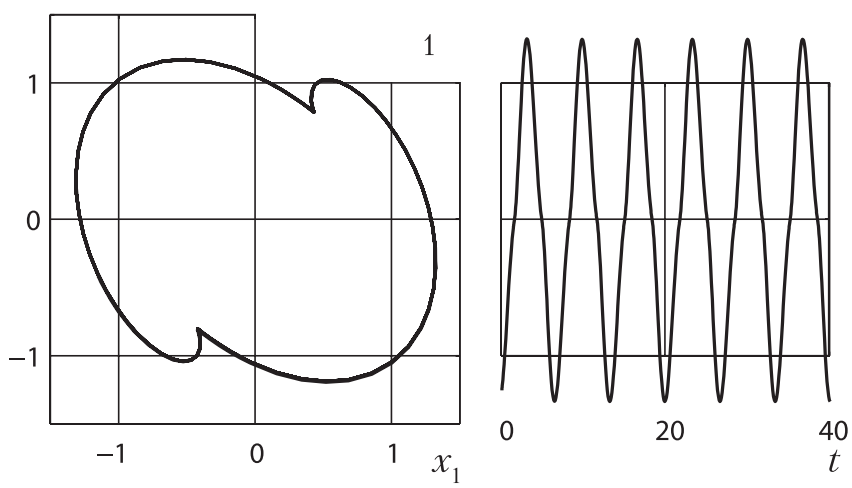

Fig. 34.

solution and

$$
\sigma(t)=\mathbf{r}^{*} \mathbf{x}(t) \approx \cos t
$$

By Theorem 9 for small $\varepsilon>0$, system (111) has the periodic solution of the form

$$
\begin{aligned}
& x_{1}(t)=\cos t+O(\varepsilon), \\
& x_{2}(t)=\sin t+O(\varepsilon), \\
& x_{3}(t)=O(\varepsilon) .
\end{aligned}
$$

Further, using the computational procedure, one can obtain periodic solutions of systems (104) for

$$
\begin{gathered}
\varphi_{j}(\sigma)=\varepsilon_{j} \varphi(\sigma), \quad \varepsilon_{1}=0.1, \\
\varepsilon_{2}=0.3, \quad \varepsilon_{3}=0.7, \quad \varepsilon_{4}=1 .
\end{gathered}
$$

In Fig. 35 are shown projections of thus computed periodic solutions on the plane $\left\{x_{1}, x_{2}\right\}$. For these periodic solutions, the graph of $\sigma(t)=x_{1}(t)+$ $x_{3}(t)$ is also given. For $\varepsilon_{3}=0.7$ and $\varepsilon_{4}=1$, the output $\sigma(t)$ is not harmonic and in this case the filter hypothesis is not valid. Therefore, here it is impossible, in principle, to justify standard describing function method by means of the filter hypothesis.

Now we give an example of the disappearance of periodic solutions with increasing parameter $\varepsilon_{j}$.

Example 3. Suppose, $\varphi(\sigma)=-3 \sigma+4 \sigma^{3}$ and

$$
W(p)=\frac{p+1}{p^{2}+1}+\frac{1}{p+1} .
$$

In this case

$$
\begin{gathered}
\omega_{0}=1, \quad b_{1}=-1, \quad b_{2}=1, \\
\mathbf{A}_{\mathbf{3}}=-1, \quad \mathbf{b}_{\mathbf{3}}=-1, \quad \mathbf{c}_{\mathbf{3}}=1 .
\end{gathered}
$$

The standard describing function method for any $\varepsilon>0$ gives the existence of periodic solution and $\sigma(t)=x_{1}(t)+x_{3}(t) \approx \cos t$. By Theorem 9 for small $\varepsilon>0$, one can obtain the periodic solution of the form (118). With increasing $\varepsilon$ the periodic solution of system (111) exists in the case when $\varepsilon \in(0, \rho)$. For $\varepsilon=\rho$, there occurs loss of stability and the vanishing of periodic solution. Figure 36 shows projections of solutions on the plane $\left\{x_{1}, x_{2}\right\}$ for $\varepsilon=0.25,0.3,0.35$.

Recall that the equilibria of system (103) satisfy the relations

$$
\begin{gathered}
\sigma_{0}+W(0) \varepsilon \varphi\left(\sigma_{0}\right)=0, \\
x_{0}=-\left(\mathbf{P}+k \mathbf{q r}^{*}\right)^{-1} \mathbf{q} \varepsilon \varphi\left(\sigma_{0}\right) .
\end{gathered}
$$

Since $W(0)=2$, the first relation yields the relation

$$
\sigma_{0}\left(1-6 \varepsilon+8 \varepsilon \sigma_{0}^{2}\right)=0 \text {. }
$$



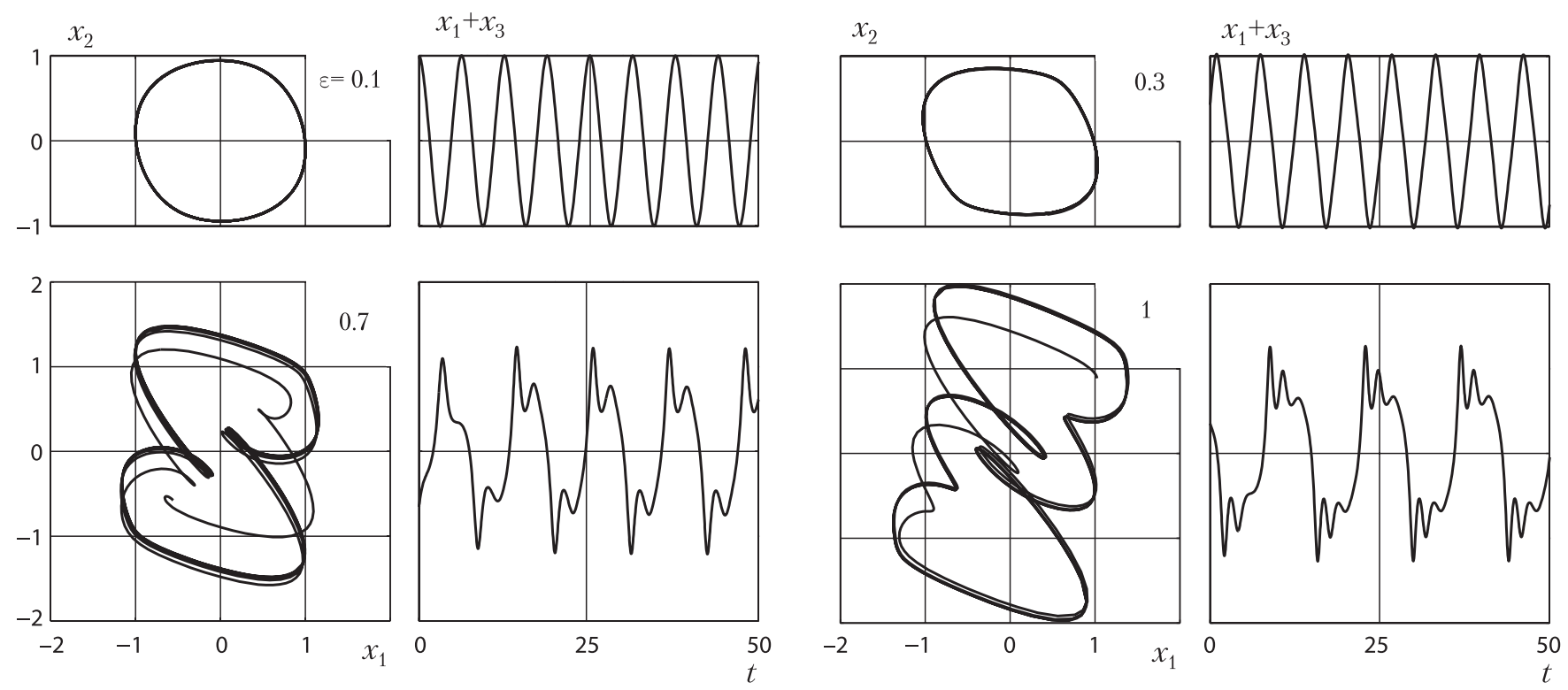

Fig. 35.

This implies that for $\varepsilon<1 / 6$ the considered system has only one zero equilibrium. For $\varepsilon>1 / 6$ the system has three equilibria

$$
\sigma_{0}=0, \quad \sigma_{0}= \pm \sqrt{\frac{6 \varepsilon-1}{8 \varepsilon}} .
$$

They are unstable for $\varphi^{\prime}\left(\sigma_{0}\right)<0$ and are stable for $\varphi^{\prime}\left(\sigma_{0}\right)>0$. Consequently, the zero equilibrium is always unstable and the nonzero one is unstable for $\varepsilon<1 / 4$ and is stable for $\varepsilon>1 / 4$. Here, the considered periodic solution is attracted to one of these equilibria. The standard describing function method overlooks all these qualitative changes in the phase space of the considered system.

\subsubsection{Poincaré map for harmonic linearization in the critical case}

As was shown in the critical case, where the conditions of Aizerman's or Kalman's conjecture are satisfied, the standard describing function method gives incorrect results. But it is possible to modify and justify it for the nonlinearities of special types.

Consider system (106)

$$
\begin{aligned}
& \dot{y}_{1}=-\omega_{0} y_{2}+b_{1} \varphi^{0}\left(y_{1}+\mathbf{c}_{3}{ }^{*} \mathbf{y}_{3}\right), \\
& \dot{y}_{2}=\omega_{0} y_{1}+b_{2} \varphi^{0}\left(y_{1}+\mathbf{c}_{3}{ }^{*} \mathbf{y}_{3}\right), \\
& \dot{\mathbf{y}}_{3}=\mathbf{A}_{3} \mathbf{y}_{3}+\mathbf{b}_{3} \varphi^{0}\left(y_{1}+\mathbf{c}_{3}{ }^{*} \mathbf{y}_{3}\right),
\end{aligned}
$$
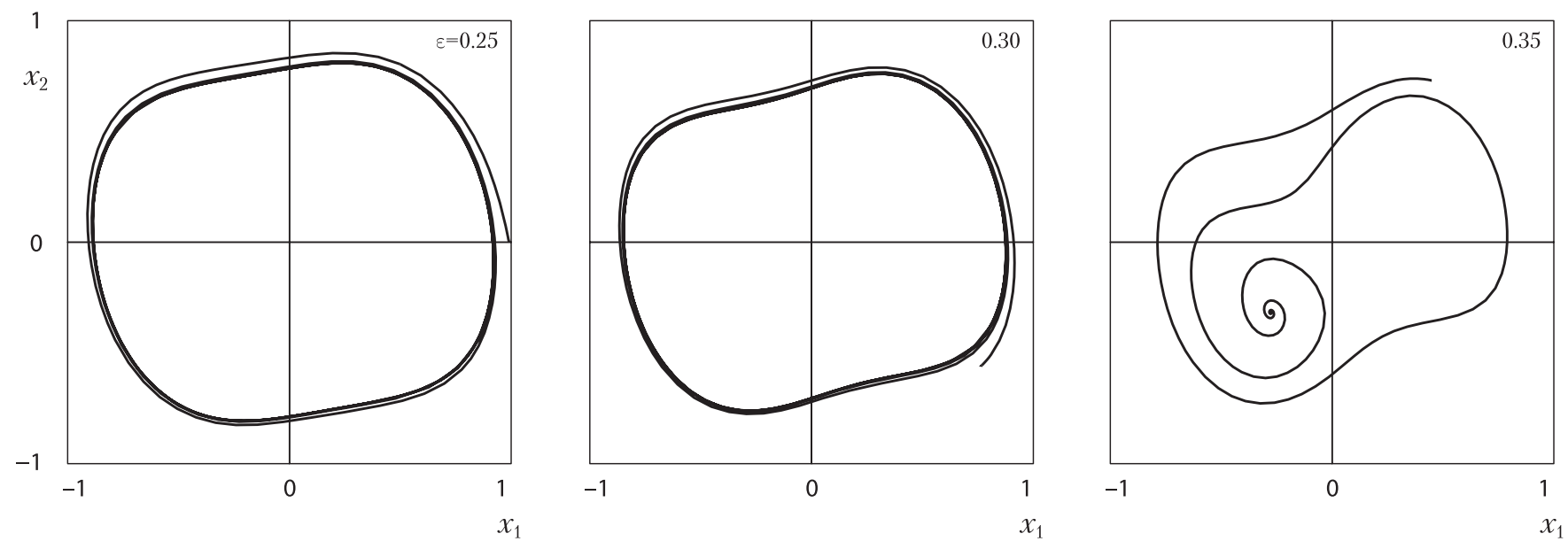

Fig. 36. Loss of stability. 
where the nonlinearity $\varphi^{0}(\sigma)$ is of special form

$$
\varphi^{0}(\sigma)= \begin{cases}\varphi_{1}(\sigma), & \forall|\sigma| \leq \varepsilon, \\ \varepsilon^{3} \varphi_{2}(\sigma), & \forall|\sigma|>\varepsilon .\end{cases}
$$

Here $\varphi_{1}(\sigma)$ is a piecewise-differentiable function and $\varphi_{2}(\sigma)$ is a differentiable function, for which the following conditions

$$
\begin{aligned}
& \left|\varphi_{1}(\sigma)\right| \leq \mu|\sigma|, \\
& \int_{-\varepsilon}^{\varepsilon}\left(b_{2}\left(\mathbf{c}_{3}{ }^{*} \mathbf{b}_{3}+b_{1}\right) \varphi_{1}(\sigma)+b_{1} \omega_{0} \sigma\right) \varphi_{1}(\sigma) d \sigma \\
& \quad=L \varepsilon^{3}+O\left(\varepsilon^{4}\right)
\end{aligned}
$$

are valid. Here $\mu>0$ and $L$ are certain numbers. Further, without loss of generality, it can be assumed that

$$
\varphi_{2}(\sigma)=0 \quad \forall \sigma \in[-\varepsilon, \varepsilon] .
$$

Such conditions are valid, for example, for the following nonlinearity

$$
\varphi^{0}(\sigma)= \begin{cases}\mu \sigma, & \forall|\sigma| \leq \varepsilon, \\ M \varepsilon^{3} \operatorname{sign}(\sigma), & \forall|\sigma|>\varepsilon,\end{cases}
$$

where $M$ is a certain positive number.

Consider in phase space of nonlinear system (124) the set

$$
\begin{gathered}
\Omega=\left\{y_{1}+\mathbf{c}_{\mathbf{3}}{ }^{*} \mathbf{y}_{3}=0, y_{2} \in\left[-a_{1},-a_{2}\right],\right. \\
\left.\left|\mathbf{y}_{3}\right| \leq D \varepsilon^{2}\right\} .
\end{gathered}
$$

Here $a_{1,2}$ are certain positive numbers, the number $D$ is defined by the following assertion.

Lemma 12. For solutions of system (124) with initial data from $\Omega$, the following representation

$$
\begin{aligned}
y_{1}(t) & =-\sin \left(\omega_{0} t\right) y_{2}(0)+O\left(\varepsilon^{2}\right), \\
y_{2}(t) & =\cos \left(\omega_{0} t\right) y_{2}(0)+O\left(\varepsilon^{2}\right), \\
\mathbf{y}_{3}(t) & =\exp \left(\mathbf{A}_{3} t\right) \mathbf{y}_{3}(0)+\mathbf{O}_{\mathbf{n}-\mathbf{2}}\left(\varepsilon^{2}\right) \\
& =\mathbf{O}_{\mathbf{n}-\mathbf{2}}\left(\varepsilon^{2}\right)
\end{aligned}
$$

is valid. In addition, there exist numbers $D_{1} \geq D>$ 0 such that if for small enough $\varepsilon>0$ the inequality

$$
\left|\mathbf{y}_{3}(0)\right| \leq D \varepsilon^{2},
$$

is satisfied, then

$$
\left|\mathbf{y}_{3}(T)\right| \leq D \varepsilon^{2}
$$

and

$$
\left|\mathbf{y}_{3}(t)\right| \leq D_{1} \varepsilon^{2}, \quad \forall t \in[0, T] .
$$

Proof. Represent a solution of the system in integral form:

$$
\begin{aligned}
\left(\begin{array}{l}
y_{1}(t) \\
y_{2}(t) \\
\mathbf{y}_{3}(t)
\end{array}\right) & \\
= & \left(\begin{array}{ccc}
\cos \left(\omega_{0} t\right) & -\sin \left(\omega_{0} t\right) & \mathbf{0} \\
\sin \left(\omega_{0} t\right) & \cos \left(\omega_{0} t\right) & \mathbf{0} \\
\mathbf{0} & \mathbf{0} & \exp \left(\mathbf{A}_{3}\left(t-t_{0}\right)\right)
\end{array}\right) \\
& \times\left(\begin{array}{c}
y_{1}\left(t_{0}\right) \\
y_{2}\left(t_{0}\right) \\
\mathbf{y}_{3}\left(t_{0}\right)
\end{array}\right)+\mathbf{I}\left(t_{0}, t\right) .
\end{aligned}
$$

Here

$$
\begin{gathered}
\mathbf{I}\left(t_{0}, t\right)=\int_{t_{0}}^{t} \exp (\mathbf{A}(t-\tau)) \mathbf{b} \varphi^{0}(\sigma(\tau)) d \tau, \\
\mathbf{A}=\left(\begin{array}{ccc}
0 & -\omega_{0} & 0 \\
\omega_{0} & 0 & 0 \\
0 & 0 & \mathbf{A}_{3}
\end{array}\right), \quad \mathbf{b}=\left(\begin{array}{l}
b_{1} \\
b_{2} \\
\mathbf{b}_{3}
\end{array}\right) .
\end{gathered}
$$

From condition (107) and the form of nonlinearity $\varphi^{0}$, one obtains the estimate

$$
\begin{array}{r}
\left|\exp \left(\mathbf{A}_{3}\left(t-t_{0}\right)\right)\right|<1, \quad\left|\mathbf{I}\left(t_{0}, t\right)\right|=O\left(\varepsilon^{2}\right), \\
0 \leq t_{0}<t \leq T .
\end{array}
$$

Then a number $D>0$ can be chosen such that $\left|\exp \left(\mathbf{A}_{3} T\right)\right| D+E<D$, where $E$ is defined from the condition $\sup _{t \in[0, T]}|\mathbf{I}(0, t)| \leq E \varepsilon^{2}$ and depends on $|\mathbf{b}|$ and the form of function $\varphi^{0}$.

Consider for trajectories of system (124), a Poincaré map $F$ of the set $\Omega$ :

$$
F\left\|\begin{array}{l}
y_{1}(0) \\
y_{2}(0) \\
\mathbf{y}_{3}(0)
\end{array}\right\|=\left\|\begin{array}{l}
y_{1}(T) \\
y_{2}(T) \\
\mathbf{y}_{3}(T)
\end{array}\right\|
$$

Here, $T$ is a positive number such that

$$
y_{1}(T)+\mathbf{c}_{\mathbf{3}}{ }^{*} \mathbf{y}_{3}(T)=0, \quad y_{2}(T)<0
$$

and the relations

$$
y_{1}(t)+\mathbf{c}_{3}{ }^{*} \mathbf{y}_{3}(t)=0, \quad y_{2}(t)<0, \quad \forall t \in(0, T)
$$

are not satisfied. Introduce a describing function

$$
\Phi(a)=\int_{0}^{2 \pi / \omega_{0}} \varphi_{2}\left(a \sin \left(\omega_{0} t\right)\right) \sin \left(\omega_{0} t\right) d t .
$$


Theorem 11 [Leonov \& Kuznetsov, 2011a]. If the inequalities

$$
b_{1} \Phi\left(a_{2}\right)>-\frac{2}{\omega_{0}^{2} a_{2}^{2}} L, \quad b_{1} \Phi\left(a_{1}\right)<-\frac{2}{\omega_{0}^{2} a_{1}^{2}} L
$$

are valid, then for sufficiently small $\varepsilon>0$, Poincaré map (135) of the set $\Omega$ is mapped to itself: $F \Omega \subset \Omega$.

In this case, by Brouwer's fixed point theorem, one can formulate the following

Corollary 4.3. If inequalities (137) are satisfied, then for sufficiently small $\varepsilon>0$ system (124) has a periodic solution with initial data from $\Omega$. This solution is stable in a sense that its neighborhood $\Omega$ is mapped in itself: $F \Omega \subset \Omega$.

Theorem 12. Suppose that there exists a number $a_{0}>0,-a_{0} \neq v_{i}$ such that the conditions

$$
b_{1} \Phi\left(a_{0}\right)=-\frac{2}{\omega_{0}^{2} a_{0}^{2}} L,\left.\quad b_{1} \frac{d \Phi(a)}{d a}\right|_{a=a_{0}}<\frac{4}{\omega_{0}^{2} a_{0}^{3}} L
$$

are satisfied. Then for sufficiently small $\varepsilon>0$, system (124) has the periodic solution of the form (130) with the initial data

$$
\begin{aligned}
& y_{1}(0)=O\left(\varepsilon^{2}\right), \\
& y_{2}(0)=-a_{0}+O(\varepsilon), \\
& \mathbf{y}_{3}(0)=\mathbf{O}_{\mathbf{n}-\mathbf{2}}\left(\varepsilon^{2}\right)
\end{aligned}
$$

and with the period

$$
T=\frac{2 \pi}{\omega_{0}}+O\left(\varepsilon^{2}\right) .
$$

Corollary 4.4. For nonlinearity (128), one obtains $L=\frac{2}{3}\left(b_{2}\left(\mathbf{c}_{3}{ }^{*} \mathbf{b}_{3}+b_{1}\right) \mu+b_{1} \omega_{0}\right) \mu, \quad \Phi\left(a_{0}\right)=M \frac{4}{\omega_{0}}$, and relation (139) results in

$$
\begin{aligned}
y_{1}(0)= & O\left(\varepsilon^{2}\right), \\
y_{2}(0)= & -\sqrt{-\frac{\mu}{3 \omega_{0} b_{1} M}\left(b_{2}\left(\mathbf{c}_{\mathbf{3}}{ }^{*} \mathbf{b}_{3}+b_{1}\right) \mu+b_{1} \omega_{0}\right)} \\
& +O(\varepsilon), \\
\mathbf{y}_{3}(0)= & \mathbf{O}_{\mathbf{n}-\mathbf{2}}\left(\varepsilon^{2}\right) .
\end{aligned}
$$

By relations (110), Theorem 12 can be reformulated in the following way.
Theorem 13. Let there exist a number $a_{0}>$ $0,-a_{0} \neq v_{i}$ such that the conditions

$$
\eta \Phi\left(a_{0}\right)=\frac{2}{\omega_{0}^{2} a_{0}^{2}} L,\left.\quad \eta \frac{d \Phi(a)}{d a}\right|_{a=a_{0}}>-\frac{4}{\omega_{0}^{2} a_{0}^{3}} L
$$

are satisfied. Then for sufficiently small $\varepsilon>0$, system (103) with transfer function (108) and nonlinearity (125) has T-periodic solution such that

$$
\begin{aligned}
\mathbf{r}^{*} \mathbf{x}(t) & =a_{0} \sin \left(\omega_{0} t\right)+O\left(\varepsilon^{2}\right), \\
T & =\frac{2 \pi}{\omega_{0}}+O\left(\varepsilon^{2}\right) .
\end{aligned}
$$

Here by (110), L is defined from the relation

$$
\begin{aligned}
& \int_{-\varepsilon}^{\varepsilon}\left(\frac{\theta}{\omega_{0}} \mathbf{r}^{*} \mathbf{q} \varphi_{1}(\sigma)-\eta \omega_{0} \sigma\right) \varphi_{1}(\sigma) d \sigma \\
& =L \varepsilon^{3}+O\left(\varepsilon^{4}\right) .
\end{aligned}
$$

A sketch of proof of Theorem 12. The proof of the theorem is based on the following lemmas.

By the nonlinearity $\varphi^{0}$ and representation of solutions (130), for the output of system (124) and a derivative of output, we can obtain the expressions

$$
\begin{aligned}
\sigma(t) & =y_{1}(t)+\mathbf{c}_{\mathbf{3}}{ }^{*} \mathbf{y}_{3}(t) \\
& =-\sin \left(\omega_{0} t\right) y_{2}(0)+O\left(\varepsilon^{2}\right), \\
\dot{\sigma}(t) & =\dot{y}_{1}(t)+\mathbf{c}_{\mathbf{3}}{ }^{*} \dot{\mathbf{y}}_{3}(t) \\
& =-\omega_{0} \cos \left(\omega_{0} t\right) y_{2}(0)+O(\varepsilon) .
\end{aligned}
$$

Hence $|\dot{\sigma}(\tau)|>\kappa>0$ for $|\sigma(\tau)| \leq \varepsilon$. From (143) and (130) it follows that there exist numbers

$$
0=\tau_{0}<\tau_{1}<\tau_{2}<\tau_{3}<\tau_{4}<\tau_{5}=T
$$

such that (see Fig. 37)

$$
\begin{array}{rll}
\tau_{1}: & \forall t \in\left(0, \tau_{1}\right) \sigma(t) \in(0, \varepsilon), & \sigma\left(\tau_{1}\right)=\varepsilon ; \\
\tau_{2}: & \forall t \in\left(\tau_{1}, \tau_{2}\right) \sigma(t)>\varepsilon, & \sigma\left(\tau_{2}\right)=\varepsilon ; \\
\tau_{3}: & \forall t \in\left(\tau_{2}, \tau_{3}\right) \sigma(t) \in(-\varepsilon, \varepsilon), & \sigma\left(\tau_{3}\right)=-\varepsilon ; \\
\tau_{4}: & \forall t \in\left(\tau_{3}, \tau_{4}\right) \sigma(t)<-\varepsilon, & \sigma\left(\tau_{4}\right)=-\varepsilon ; \\
\tau_{5}=T: & \forall t \in\left(\tau_{4}, T\right) \sigma(t) \in(-\varepsilon, 0), & \sigma(T)=0
\end{array}
$$

are valid. 


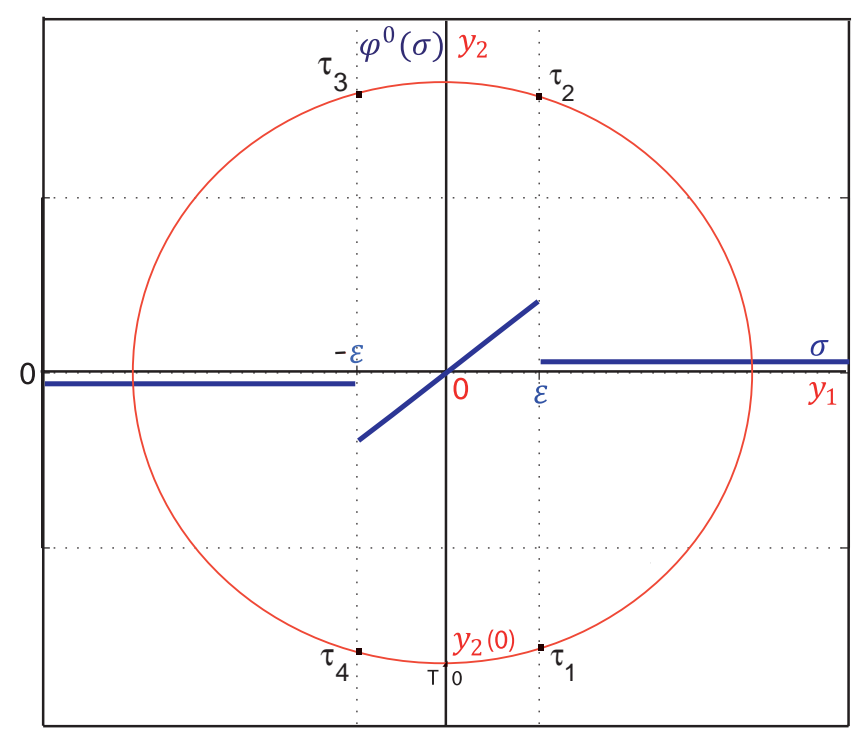

Fig. 37. Projection of solution on the plane $\left(y_{1}, y_{2}\right)$ and the nonlinearity $\varphi^{0}(\sigma)$ of the form (128).

By the first relation of (143), one can formulate the following assertion.

Lemma 13. The following relations

$$
\begin{aligned}
\tau_{1} & =\frac{\varepsilon}{\omega_{0}\left|y_{2}(0)\right|}+O\left(\varepsilon^{2}\right), \\
\tau_{2}-\tau_{1} & =\frac{\pi}{\omega_{0}}-\frac{2 \varepsilon}{\omega_{0}\left|y_{2}(0)\right|}+O\left(\varepsilon^{2}\right), \\
\tau_{3}-\tau_{2} & =\frac{2 \varepsilon}{\omega_{0}\left|y_{2}(0)\right|}+O\left(\varepsilon^{2}\right), \\
\tau_{4}-\tau_{3} & =\frac{\pi}{\omega_{0}}-\frac{2 \varepsilon}{\omega_{0}\left|y_{2}(0)\right|}+O\left(\varepsilon^{2}\right), \\
T-\tau_{4} & =\frac{\varepsilon}{\omega_{0}\left|y_{2}(0)\right|}+O\left(\varepsilon^{2}\right)
\end{aligned}
$$

are valid.

Lemma 14. The following estimate

$$
\begin{aligned}
\int_{0}^{2 \pi / \omega_{0}} \varphi_{2}(\sigma(t)) d t \\
\quad=\int_{0}^{2 \pi / \omega_{0}} \varphi_{2}\left(-\sin \left(\omega_{0} t\right) y_{2}(0)\right) d t+O(\varepsilon)
\end{aligned}
$$

is satisfied.

Proof. From the continuity of $\sigma(t)$ and the boundedness of the function $\varphi_{2}(\sigma)$ on finite intervals, it follows that for fixed $\tau$ and $m$ the following relation

$$
\int_{\tau-m \varepsilon}^{\tau+m \varepsilon} \varphi_{2}(\sigma(t)) d t=O(\varepsilon)
$$

is valid.

Suppose, $\pm y_{2}(0) \neq \nu_{i}$. Then if $-\sin \left(\omega_{0} \tau\right) \times$ $y_{2}(0)=\nu_{i}$, one can obtain $\omega_{0} \tau \neq \frac{\pi}{2}+\pi k$. Hence by (143) for sufficiently small $\varepsilon$ the estimate $|\dot{\sigma}(\tau)|>\kappa>0$ is satisfied. Then, for all time intervals $\left[t_{j}, t_{j+1}\right]$ outside the neighborhoods $(\tau-m \varepsilon$, $\tau+m \varepsilon$ ) of time $\tau$, where

$$
-\sin \left(\omega_{0} \tau\right) y_{2}(0)=\nu_{i},
$$

for sufficiently small $\varepsilon$ it can be found that

$$
\begin{aligned}
& -\sin \left(\omega_{0} t\right) y_{2}(0) \neq \nu_{i} \\
& \sigma(t)=-\sin \left(\omega_{0} t\right) y_{2}(0)+O\left(\varepsilon^{2}\right) \neq\left|\nu_{i}\right| \\
& \forall t \in\left[t_{j}, t_{j+1}\right] .
\end{aligned}
$$

Suppose, $\pm y_{2}(0)=\nu_{i}$. Then, for all time intervals $\left[t_{j}, t_{j+1}\right]$ outside the neighborhoods $(\tau-m \varepsilon, \tau+$ $m \varepsilon$ ) of the points $\omega_{0} \tau=\frac{\pi}{2}+\pi k$ for sufficiently small $\varepsilon$ one can obtain that

$$
\begin{aligned}
|\sigma(t)| \leq & \left|-\sin \left(\omega_{0} t\right) y_{2}(0)\right|+D_{1} \varepsilon^{2} \\
= & \left|\nu_{i}\right|\left(1-\frac{1}{2}\left(\omega_{0} m \varepsilon\right)^{2}+O\left(\varepsilon^{3}\right)\right) \\
& +D_{1} \varepsilon^{2}, \quad \forall t \in\left[t_{j}, t_{j+1}\right] .
\end{aligned}
$$

Choosing $m$ such that

$$
\left|\nu_{i}\right| \frac{1}{2}\left(\omega_{0} m\right)^{2}>D_{1}
$$

one can obtain

$$
\begin{aligned}
& -\sin \left(\omega_{0} t\right) y_{2}(0) \neq \nu_{i}, \\
& |\sigma(t)|<\left|y_{2}(0)\right|=\left|\nu_{i}\right|
\end{aligned} \quad \forall t \in\left[t_{j}, t_{j+1}\right] .
$$

Taking into account boundedness of the derivative of $\varphi_{2}(\sigma)$ on continuity intervals, from (149) and (150), one can obtain

$$
\begin{aligned}
\varphi_{2}(\sigma(t))= & \varphi_{2}\left(-\sin \left(\omega_{0} t\right) y_{2}(0)\right) \\
& +O\left(\varepsilon^{2}\right), \quad t \in\left[t_{j}, t_{j+1}\right] .
\end{aligned}
$$

The estimates (148) and (151) are uniform on $[0,2 \pi]$. This proves the lemma. 
Lemma 15. For sufficiently small $\varepsilon>0$, the estimate

$$
\begin{aligned}
y_{2}^{2}(T) & -y_{2}^{2}(0) \\
= & 2\left|y_{2}(0)\right|\left(\frac{2}{\omega_{0}^{2}\left|y_{2}(0)\right|^{2}} L+b_{1} \Phi\left(\left|y_{2}(0)\right|\right)\right) \varepsilon^{3} \\
& +O\left(\varepsilon^{4}\right)
\end{aligned}
$$

is satisfied.

Proof. Consider a function

$$
V\left(y_{1}, y_{2}\right)=y_{1}^{2}+y_{2}^{2},
$$

where $y_{1}(t)$ and $y_{2}(t)$ are solutions with initial data from $\Omega$. The derivative $V(t)=V\left(y_{1}(t), y_{2}(t)\right)$ as per system (124) is as follows

$$
\dot{V}\left(y_{1}(t), y_{2}(t)\right)=2\left(y_{1}(t) b_{1}+y_{2}(t) b_{2}\right) \varphi^{0}(\sigma(t)) .
$$

By (130) the estimate $V(T)-V(0)=y_{2}^{2}(T)-$ $y_{2}^{2}(0)+O\left(\varepsilon^{4}\right)$ can be obtained.

Let us estimate the increment

$$
\begin{aligned}
V(T)-V(0) & =\int_{0}^{T} \dot{V}(t) d t \\
& =\int_{0}^{T} 2\left(y_{1}(t) b_{1}+y_{2}(t) b_{2}\right) \varphi^{0}(\sigma(t)) d t
\end{aligned}
$$

using a representation of an integral, taken on $[0, T]$, as a sum of integrals, taken on $\left[\tau_{i}, \tau_{i+1}\right]$.

(1) By (145) for $t \in\left[\tau_{1}, \tau_{2}\right] \cup\left[\tau_{3}, \tau_{4}\right]$, from the definition of nonlinearity (125), and (154), one can define

$$
\begin{aligned}
& \int_{\left[\tau_{1}, \tau_{2}\right] \cup\left[\tau_{3}, \tau_{4}\right]} \dot{V}(t) d t \\
& \quad=\int_{\left[\tau_{1}, \tau_{2}\right] \cup\left[\tau_{3}, \tau_{4}\right]} 2\left(y_{1}(t) b_{1}+y_{2}(t) b_{2}\right) \varepsilon^{3} \varphi_{2}(\sigma(t)) d t .
\end{aligned}
$$

Taking into account (127) and the form of solutions (130), one finds

$$
\begin{aligned}
& \int_{\left[\tau_{1}, \tau_{2}\right] \cup\left[\tau_{3}, \tau_{4}\right]} \dot{V}(t) d t \\
& =\int_{0}^{2 \pi / \omega_{0}} 2\left(y_{1}(t) b_{1}+y_{2}(t) b_{2}\right) \varepsilon^{3} \varphi_{2}(\sigma(t)) d t \\
& =2 \int_{0}^{2 \pi / \omega_{0}}\left(-\sin \left(\omega_{0} t\right) y_{2}(0) b_{1}\right. \\
& \left.\quad+\cos \left(\omega_{0} t\right) y_{2}(0) b_{2}\right) \varepsilon^{3} \varphi_{2} \\
& \quad \times\left(-\sin \left(\omega_{0} t\right) y_{2}(0)+O\left(\varepsilon^{2}\right)\right) d t+O\left(\varepsilon^{4}\right) .
\end{aligned}
$$

By (147) and (136)

$$
\begin{aligned}
\int_{\left[\tau_{1}, \tau_{2}\right] \cup\left[\tau_{3}, \tau_{4}\right]} \dot{V}(t) d t \\
=-2 y_{2}(0) b_{1} \varepsilon^{3} \int_{0}^{2 \pi / \omega_{0}} \sin \left(\omega_{0} t\right) \varphi_{2} \\
\quad \times\left(-\sin \left(\omega_{0} t\right) y_{2}(0)\right) d t+O\left(\varepsilon^{4}\right) \\
=2\left|y_{2}(0)\right| b_{1} \varepsilon^{3} \Phi\left(\left|y_{2}(0)\right|\right)+O\left(\varepsilon^{4}\right) .
\end{aligned}
$$

(2) Let us perform the estimation of $t \in\left[0, \tau_{1}\right] \cup$ $\left[\tau_{2}, \tau_{3}\right] \cup\left[\tau_{4}, T\right]$. By $(130)$, for $y_{1}(t)$ and $y_{2}(t)$ the following estimates

$$
\begin{aligned}
y_{1}(t) & =\sigma(t)+O\left(\varepsilon^{2}\right), \\
y_{2}(t) & =\cos \left(\omega_{0} t\right) y_{2}(0)+O\left(\varepsilon^{2}\right) \\
& = \begin{cases}y_{2}(0)+O\left(\varepsilon^{2}\right), & t \in\left[0, \tau_{1}\right] \cup\left[\tau_{4}, T\right] \\
-y_{2}(0)+O\left(\varepsilon^{2}\right), & t \in\left[\tau_{2}, \tau_{3}\right]\end{cases}
\end{aligned}
$$

are satisfied. For the derivative $\dot{\sigma}(t)$ as per system (124), the following estimate holds:

$$
\dot{\sigma}(t)= \begin{cases}-\omega_{0} y_{2}(0)+b_{1} \varphi_{1}(\sigma(t))+\mathbf{c}_{\mathbf{3}}{ }^{*} \mathbf{b}_{3} \varphi_{1}(\sigma(t))+O\left(\varepsilon^{2}\right), & t \in\left[0, \tau_{1}\right] \cup\left[\tau_{4}, T\right] \\ \omega_{0} y_{2}(0)+b_{1} \varphi_{1}(\sigma(t))+\mathbf{c}_{\mathbf{3}}{ }^{*} \mathbf{b}_{3} \varphi_{1}(\sigma(t))+O\left(\varepsilon^{2}\right), & t \in\left[\tau_{2}, \tau_{3}\right] .\end{cases}
$$

Then by estimates (126) and (146)

$$
\dot{\sigma}(t)=-\omega_{0} \cos \left(\omega_{0} t\right)+O(\varepsilon) \neq 0 .
$$

Then the function $t(\sigma)$, inverse to the function $\sigma(t)$ on the intervals of fixed sign of $\dot{\sigma}(t)$, can be considered. The substitution of (155) in relation (153) for $\dot{V}(t)$ and the use of (156) give

$$
\int_{0}^{\tau_{1}} \dot{V}(t) d t=\int_{0}^{\varepsilon} \frac{\dot{V}(t(\sigma))}{\dot{\sigma}(t(\sigma))} d \sigma=2 \int_{0}^{\varepsilon} \frac{\left(\sigma b_{1}+y_{2}(0) b_{2}\right) \varphi_{1}(\sigma)}{-\omega_{0} y_{2}(0)+b_{1} \varphi_{1}(\sigma)+\mathbf{c}_{3}{ }^{*} \mathbf{b}_{3} \varphi_{1}(\sigma)} d \sigma+O\left(\varepsilon^{4}\right),
$$




$$
\begin{aligned}
\int_{\tau_{4}}^{T} \dot{V}(t) d t & =\int_{-\varepsilon}^{0} \frac{\dot{V}(t(\sigma))}{\dot{\sigma}(t(\sigma))} d \sigma=2 \int_{-\varepsilon}^{0} \frac{\left(\sigma b_{1}+y_{2}(0) b_{2}\right) \varphi_{1}(\sigma)}{-\omega_{0} y_{2}(0)+b_{1} \varphi_{1}(\sigma)+\mathbf{c}_{3}{ }^{*} \mathbf{b}_{3} \varphi_{1}(\sigma)} d \sigma+O\left(\varepsilon^{4}\right), \\
\int_{\tau_{2}}^{\tau_{3}} \dot{V}(t) d t & =-\int_{-\varepsilon}^{\varepsilon} \frac{\dot{V}(t(\sigma))}{\dot{\sigma}(t(\sigma))} d \sigma=-2 \int_{-\varepsilon}^{\varepsilon} \frac{\left(\sigma b_{1}-y_{2}(0) b_{2}\right) \varphi_{1}(\sigma)}{\omega_{0} y_{2}(0)+b_{1} \varphi_{1}(\sigma)+\mathbf{c}_{3}{ }^{*} \mathbf{b}_{3} \varphi_{1}(\sigma)} d \sigma+O\left(\varepsilon^{4}\right) .
\end{aligned}
$$

The summation of the relations obtained gives the following

$$
\begin{aligned}
& \int_{\left[0, \tau_{1}\right] \cup\left[\tau_{2}, \tau_{3}\right] \cup\left[\tau_{4}, T\right]} \dot{V}(t) d t \\
& \quad=2 \int_{-\varepsilon}^{\varepsilon}\left(\frac{b_{1} \sigma+b_{2} y_{2}(0)}{-\omega_{0} y_{2}(0)+\mathbf{c}_{3}{ }^{*} \mathbf{b}_{3} \varphi_{1}(\sigma)+b_{1} \varphi_{1}(\sigma)}-\frac{b_{1} \sigma-b_{2} y_{2}(0)}{\omega_{0} y_{2}(0)+\mathbf{c}_{3}{ }^{*} \mathbf{b}_{3} \varphi_{1}(\sigma)+b_{1} \varphi_{1}(\sigma)}\right) \varphi_{1}(\sigma) d \sigma+O\left(\varepsilon^{4}\right) \\
& \quad=4 \int_{-\varepsilon}^{\varepsilon} \frac{\left(b_{2}\left(\mathbf{c}_{3}{ }^{*} \mathbf{b}_{3}+b_{1}\right) \varphi_{1}(\sigma)+b_{1} \omega_{0} \sigma\right)}{\omega_{0}^{2}\left|y_{2}(0)\right|} \varphi_{1}(\sigma) d \sigma+O\left(\varepsilon^{4}\right)=\frac{4}{\omega_{0}^{2}\left|y_{2}(0)\right|} L \varepsilon^{3}+O\left(\varepsilon^{4}\right)
\end{aligned}
$$

Lemmas 12 and 15 imply that if inequalities (137) are satisfied, then the inclusion $F \Omega \subset \Omega$ occurs. By Brouwer's fixed point theorem, from this inclusion it follows that there is a fixed point of the map $F$ and, consequently, there is a periodic solution of system (124) with initial data from the set $\Omega$.

\subsection{Hidden oscillations in counterexamples to Aizerman's and Kalman's conjectures}

Let us use the proposed above algorithm for the search of periodic solutions of systems under the conditions of Aizerman's and Kalman's conjectures.

Consider a system

$$
\begin{aligned}
& \dot{x}_{1}=-\omega_{0} x_{2}+b_{1} \varphi^{j}\left(x_{1}+\mathbf{c}_{3}{ }^{*} \mathbf{x}_{3}\right) \\
& \dot{x}_{2}=\omega_{0} x_{1}+b_{2} \varphi^{j}\left(x_{1}+\mathbf{c}_{\mathbf{3}}{ }^{*} \mathbf{x}_{3}\right) \\
& \dot{\mathbf{x}}_{3}=\mathbf{A}_{3} \mathbf{x}_{3}+\mathbf{b}_{3} \varphi^{j}\left(x_{1}+\mathbf{c}_{3}{ }^{*} \mathbf{x}_{3}\right)
\end{aligned}
$$

and a finite sequence of functions

$$
\begin{array}{r}
\varphi^{j}(\sigma)= \begin{cases}\mu \sigma, & \forall|\sigma| \leq \varepsilon_{j}, \\
M \operatorname{sign}(\sigma) \varepsilon_{j}^{3}, & \forall|\sigma|>\varepsilon_{j},\end{cases} \\
\varepsilon_{j}=\frac{j}{m} \sqrt{\frac{\mu}{M}}, \quad j=1, \ldots, m,
\end{array}
$$

satisfying the condition of Aizerman's conjecture for the sector $\left(0, \mu_{2}\right)$. Here $\mu, M$ are certain positive numbers and $\mu<\mu_{2}$. Choose $m$ in such a way that the graphs of the functions $\varphi^{j}$ and $\varphi^{j+1}$ slightly differ from one another. Here, for sufficiently small $\varepsilon_{j}=\varepsilon$, according to (140), in system (157) there is a periodic solution with initial data

$$
\begin{aligned}
x_{1}(0)= & O\left(\varepsilon^{2}\right), \\
x_{2}(0)= & -\sqrt{-\frac{\mu}{3 \omega_{0} b_{1} M}\left(b_{2}\left(\mathbf{c}_{3} * \mathbf{b}_{3}+b_{1}\right) \mu+b_{1} \omega_{0}\right)} \\
& +O(\varepsilon), \\
\mathbf{x}_{3}(0)= & \mathbf{O}\left(\varepsilon^{2}\right) .
\end{aligned}
$$

At the first step of algorithm, in the case $j=1$ for sufficiently large time interval $[0, T]$ one computes the solution $\mathbf{x}^{1}(t)$ with the initial data

$$
\left(0,-\sqrt{-\frac{\mu}{3 \omega_{0} b_{1} M}\left(b_{2}\left(\mathbf{c}_{3} * \mathbf{b}_{3}+b_{1}\right) \mu+b_{1} \omega_{0}\right)}, \mathbf{0}\right) .
$$

If in process of computation the solution tends to the periodic one, then, according to the algorithm, we can compute a solution of system with $\varepsilon_{2}$, using as initial data the value $\mathbf{x}^{1}(T)$.

Suppose that by this algorithm the periodic solution $\mathbf{x}^{m}(t)$ of system (157) with the continuous nondecreasing function $\varphi^{m}(\sigma)$ ("saturation") is computed. In this case, for constructing a counterexample to Kalman's problem, two algorithms are realized.

(1) Let us organize the following computational procedure for a sequence of nonlinearities [Leonov et al., 2010a]

$$
\psi^{i}(\sigma)=\left\{\begin{array}{l}
\mu \sigma, \quad \forall|\sigma| \leq \varepsilon_{m} \\
i\left(\sigma-\operatorname{sign}(\sigma) \varepsilon_{m}\right) N \\
\quad+\operatorname{sign}(\sigma) \mu \varepsilon_{m}, \quad \forall|\sigma|>\varepsilon_{m},
\end{array}\right.
$$


where $i=0, \ldots, h, \psi^{0}(\sigma)=\varphi^{m}(\sigma)$, and $N$ is a certain positive parameter such that $h N<\mu_{2}$. Here at each step for $i=1, \ldots, h$, we can obtain strictly increasing nonlinearity and the periodic solution computed gives a certain counterexample to Kalman's problem.

For illustration of this algorithm, one can consider a system

$$
\begin{aligned}
& \dot{x}_{1}=-x_{2}-10 \varphi\left(x_{1}-10.1 x_{3}-0.1 x_{4}\right), \\
& \dot{x}_{2}=x_{1}-10.1 \varphi\left(x_{1}-10.1 x_{3}-0.1 x_{4}\right), \\
& \dot{x}_{3}=x_{4}, \\
& \dot{x}_{4}=-x_{3}-x_{4}+\varphi\left(x_{1}-10.1 x_{3}-0.1 x_{4}\right) .
\end{aligned}
$$

Here for $\varphi(\sigma)=k \sigma$ the asymptotic stability of linear system (162) occurs for $k \in(0,9.9)$ while by Theorem 11 for the nonlinearity $\varphi(\sigma)=$ $\varphi^{0}(\sigma)$ with sufficiently small $\varepsilon$, there is a periodic solution.

The above algorithm allows one to sequentially compute periodic solutions. ${ }^{2}$ Suppose, $\mu=M=1$ (in this case, nonlinearity (158) lies in the linear stability sector), $\varepsilon_{1}=0.1, \varepsilon_{2}=0.2, \ldots, \varepsilon_{10}=1$. For $j=1, \ldots, 10$, one sequentially constructs solutions of system (162), assuming that the nonlinearity $\varphi(\sigma)$ is equal to $\varphi^{j}(\sigma)$ according to (158). Here, for all $j=1, \ldots, 10$, there are periodic solutions.

At the first step for $j=0$, according to (159), the initial data of periodic solution are the following

$$
x_{1}(0)=x_{3}(0)=x_{4}(0)=0, \quad x_{2}(0)=-1.7513 .
$$

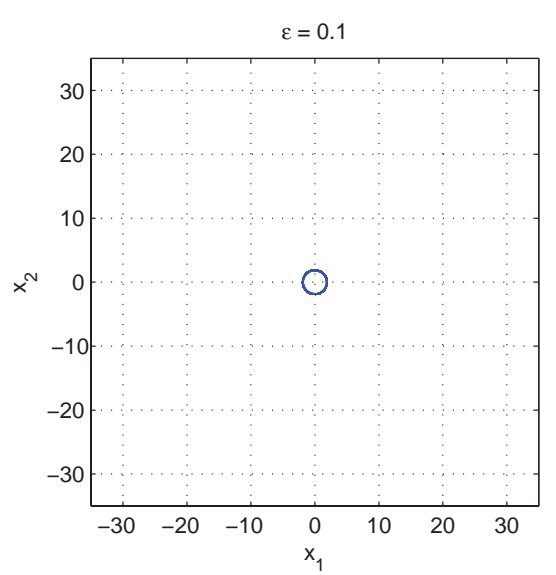

Compute the trajectory $\mathbf{x}^{1}(t)$ with the abovementioned initial data on large time interval. The last point $\mathbf{x}^{1}(T)$ of the computed trajectory is taken as the initial data for the computation of periodic solution for $j=2$.

The projection of solution trajectory on the plane $\left(x_{1}, x_{2}\right)$ and the output of system $\sigma(t)=$ $x_{1}(t)-10.1 x_{3}(t)-0.1 x_{4}(t)$ for $j=1$ are shown in Fig. 38. Here, one observes that after a transient process the numerical procedure reaches a periodic solution.

Proceeding for $j=2, \ldots, 10$, one can compute sequentially (Figs. 39-46) a periodic solution of system (162). For $\varepsilon_{10}=1$, this periodic solution is shown in Fig. 47.

Note that if in place of sequential increasing $\varepsilon_{j}$, a solution is computed with initial data (160) for $\varepsilon=1$, then this solution is attracted to zero.

Continue sequential construction of periodic solutions for system (162), with the nonlinearity $\varphi(\sigma)$ being changed, according to (161), to the strictly increasing function $\psi^{i}(\sigma)$ with $\mu=1, \varepsilon_{m}=$ $1, N=0.01$, for $i=1, \ldots, 5$. For $i=1$ and 5 the periodic solutions obtained are shown in Figs. 48 and 49. In computing a solution with $i=6$, a periodic solution vanishes (Fig. 50).

(2) Now in place of the consideration of the functions $\psi^{i}$, we construct periodic solutions for system (162) by the changes of the nonlinearity $\varphi^{10}(\sigma)$ to

$$
\theta^{i}(\sigma)=\varphi^{10}(\sigma)+\left(\tanh (\sigma)-\varphi^{10}(\sigma)\right) \frac{i}{m},
$$

Fig. 38. A projection of trajectory on the plane $\left(x_{1}, x_{2}\right)$ and the output of system for $\varepsilon_{1}=0.1$; the linear stability sector and the nonlinearity $\varphi^{1}(\sigma)$.

\footnotetext{
${ }^{2}$ Standard Matlab tools were used for computation of trajectories.
} 

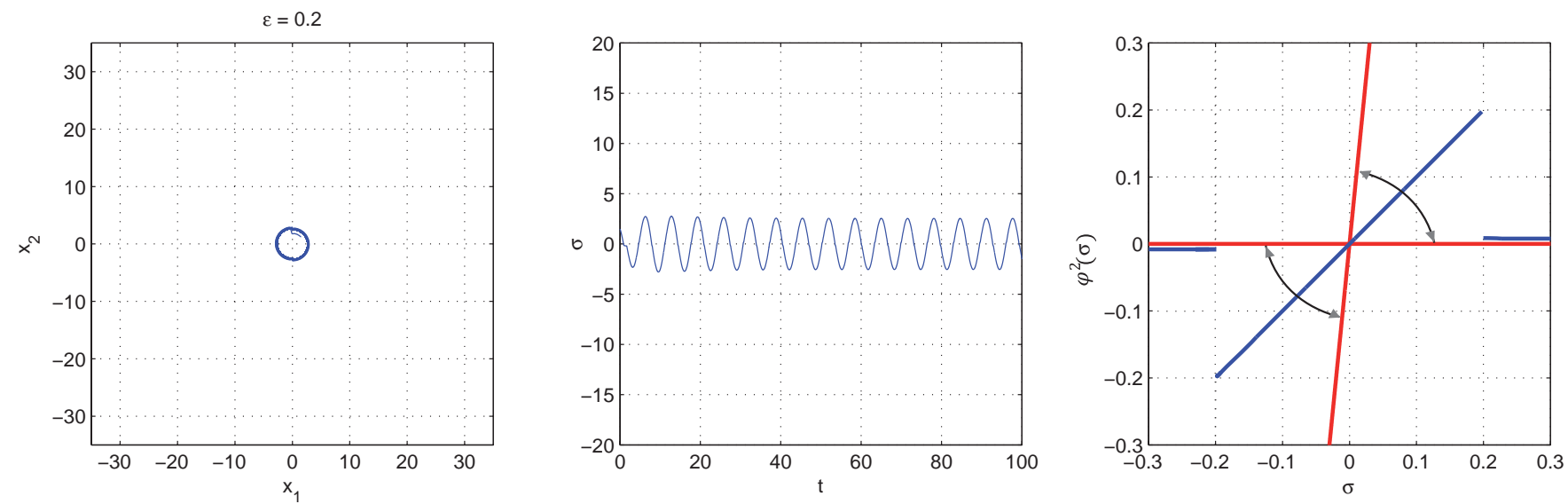

Fig. 39. A projection of trajectory on the plane $\left(x_{1}, x_{2}\right)$ and the output of system for $\varepsilon_{2}=0.2$; the linear stability sector and the nonlinearity $\varphi^{2}(\sigma)$.
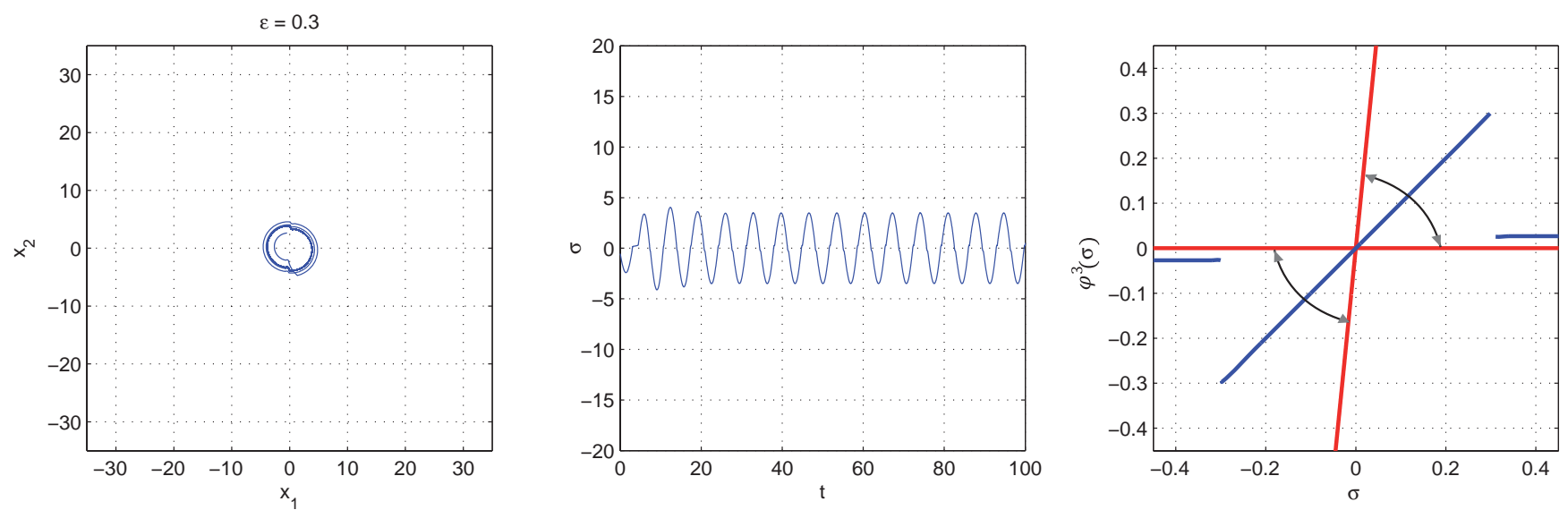

Fig. 40. A projection of trajectory on the plane $\left(x_{1}, x_{2}\right)$ and the output of system for $\varepsilon_{3}=0.3$; the linear stability sector and the nonlinearity $\varphi^{3}(\sigma)$.
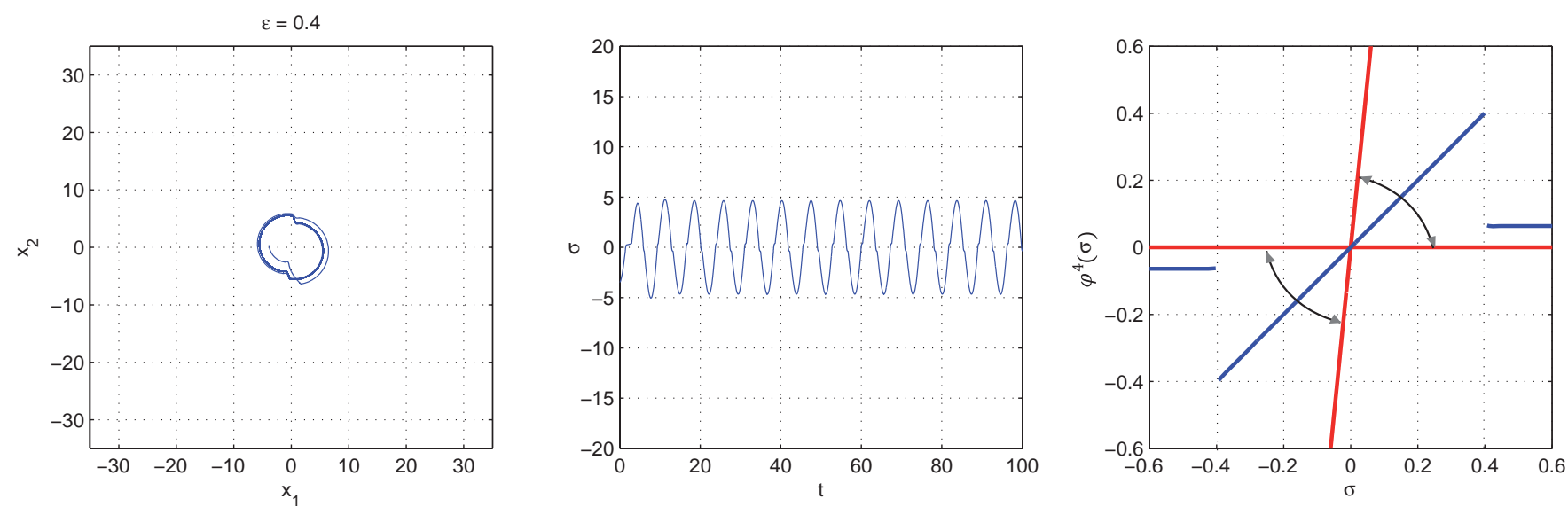

Fig. 41. A projection of trajectory on the plane $\left(x_{1}, x_{2}\right)$ and the output of system for $\varepsilon_{4}=0.4$; the linear stability sector and the nonlinearity $\varphi^{4}(\sigma)$. 

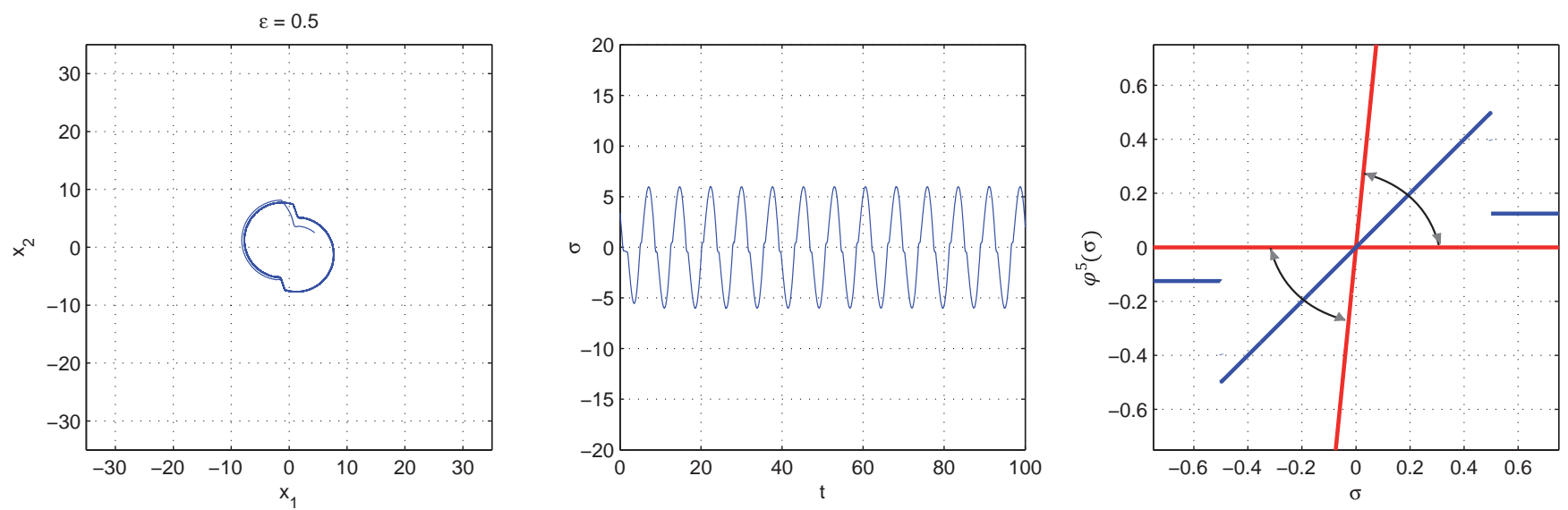

Fig. 42. A projection of trajectory on the plane $\left(x_{1}, x_{2}\right)$ and the output of system for $\varepsilon_{5}=0.5$; the linear stability sector and the nonlinearity $\varphi^{5}(\sigma)$.
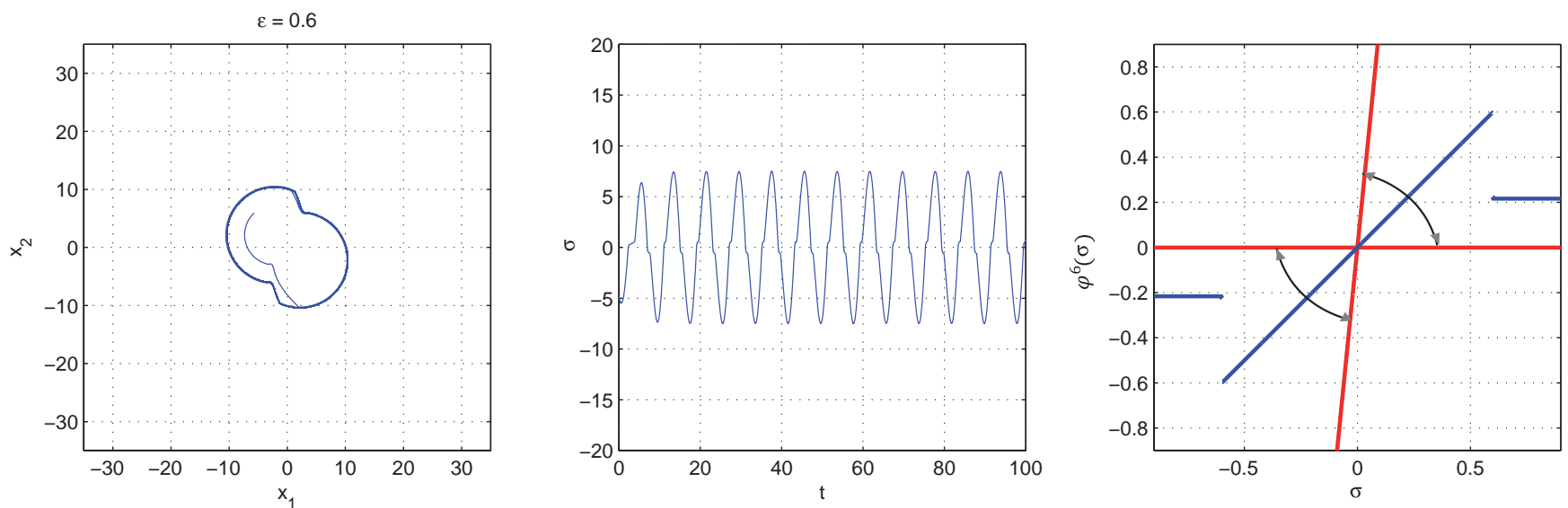

Fig. 43. A projection of trajectory on the plane $\left(x_{1}, x_{2}\right)$ and a system output for $\varepsilon_{6}=0.6$; the linear stability sector and the nonlinearity $\varphi^{6}(\sigma)$.
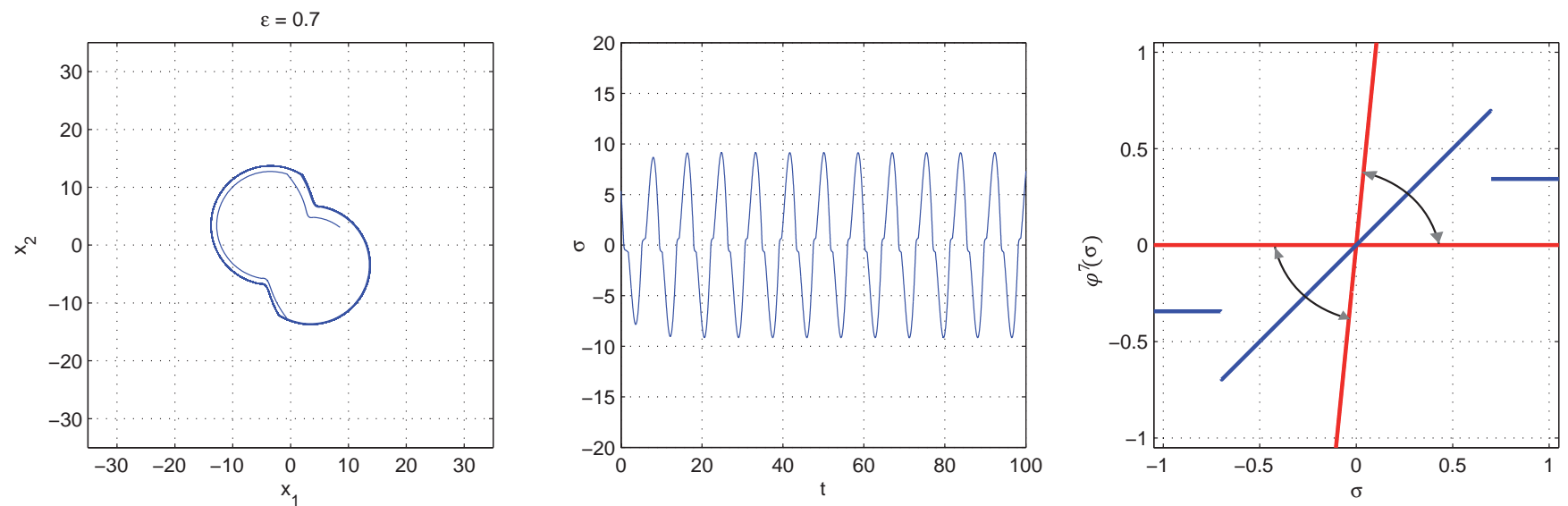

Fig. 44. A projection of trajectory on the plane $\left(x_{1}, x_{2}\right)$ and the output of system for $\varepsilon_{7}=0.7$; the linear stability sector and the nonlinearity $\varphi^{7}(\sigma)$. 

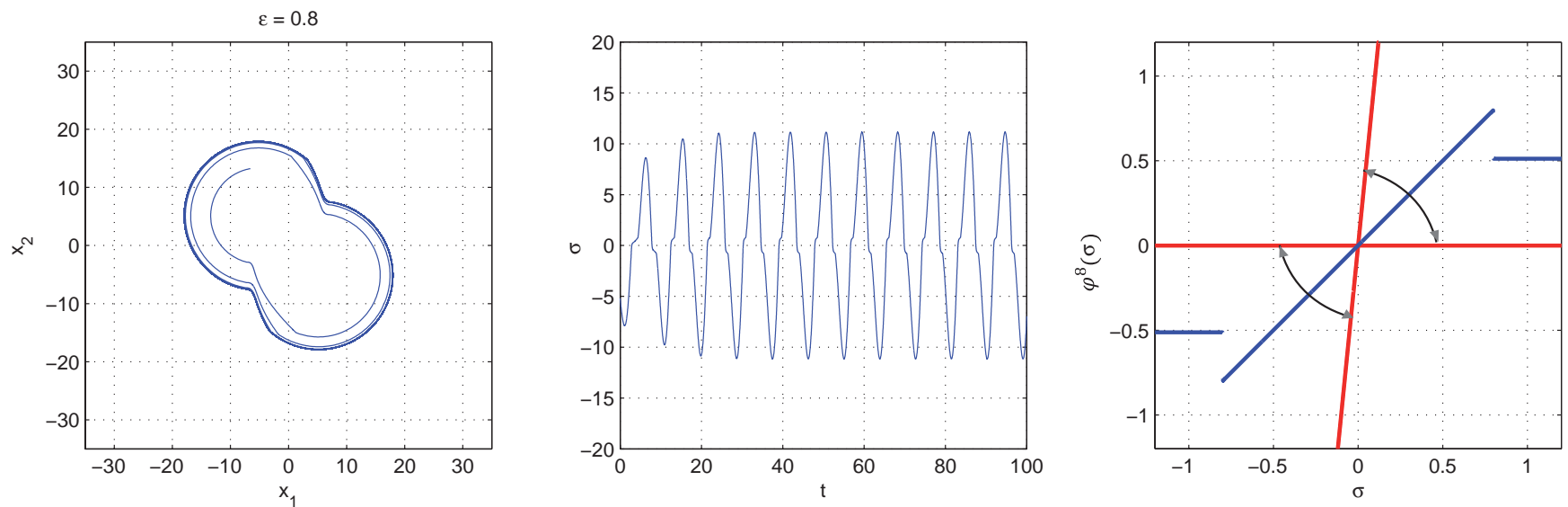

Fig. 45. A projection of trajectory on the plane $\left(x_{1}, x_{2}\right)$ and the output of system for $\varepsilon_{8}=0.8$; the linear stability sector and the nonlinearity $\varphi^{8}(\sigma)$.
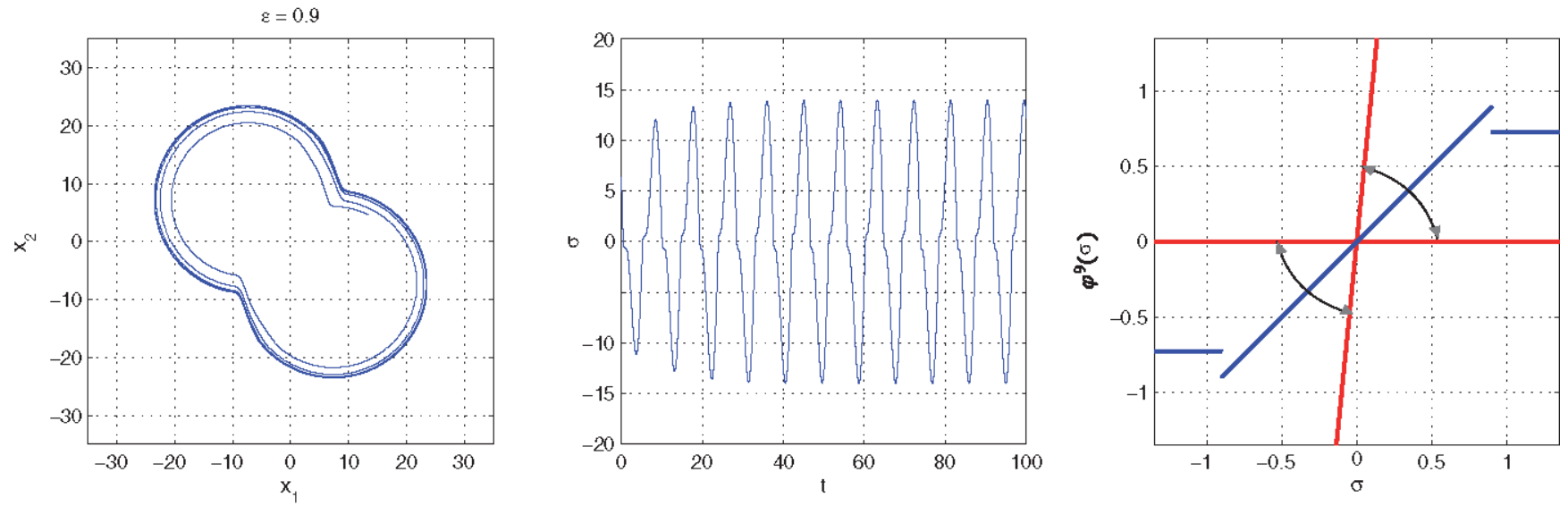

Fig. 46. A projection of trajectory on the plane $\left(x_{1}, x_{2}\right)$ and the output of system for $\varepsilon_{9}=0.9$; the linear stability sector and the nonlinearity $\varphi^{9}(\sigma)$.
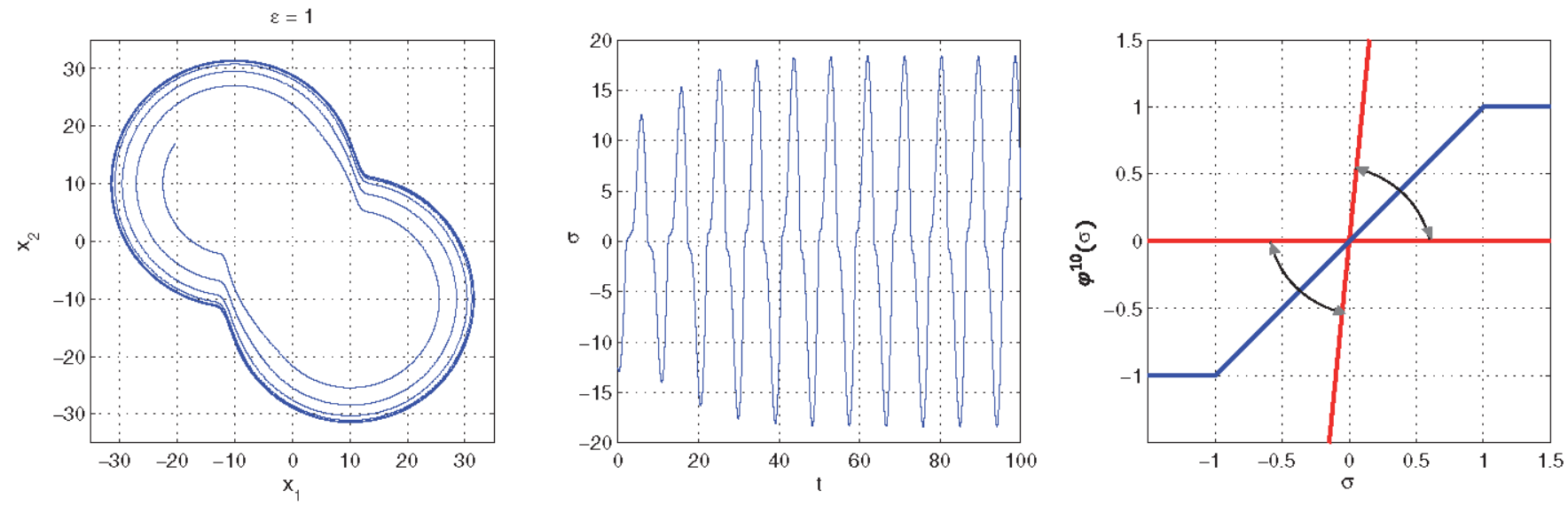

Fig. 47. A projection of trajectory on the plane $\left(x_{1}, x_{2}\right)$ and the output of system for $\varepsilon_{10}=1$; the linear stability sector and the nonlinearity $\operatorname{sat}(\sigma)=\varphi^{10}(\sigma)$. 

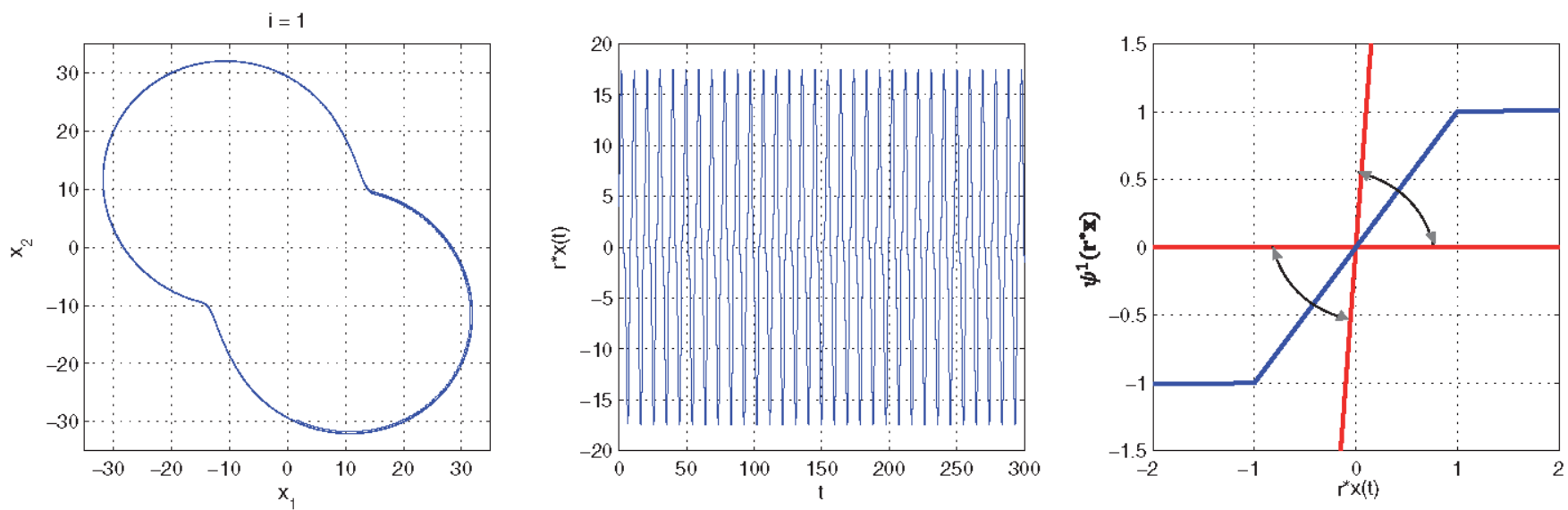

Fig. 48. A projection of trajectory on the plane $\left(x_{1}, x_{2}\right)$ and the output of system for $i=1$; the linear stability sector and the nonlinearity $\psi^{1}(\sigma)$.
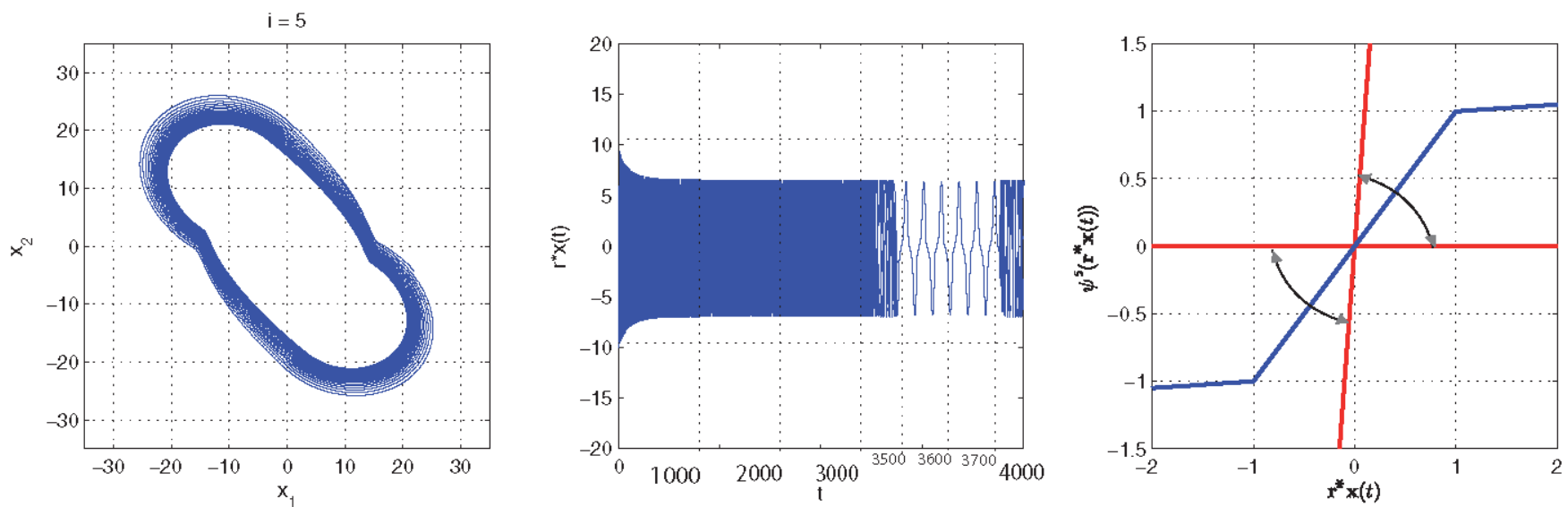

Fig. 49. A projection of trajectory on the plane $\left(x_{1}, x_{2}\right)$ and the output of system for $i=5$; the linear stability sector and the nonlinearity $\psi^{5}(\sigma)$.
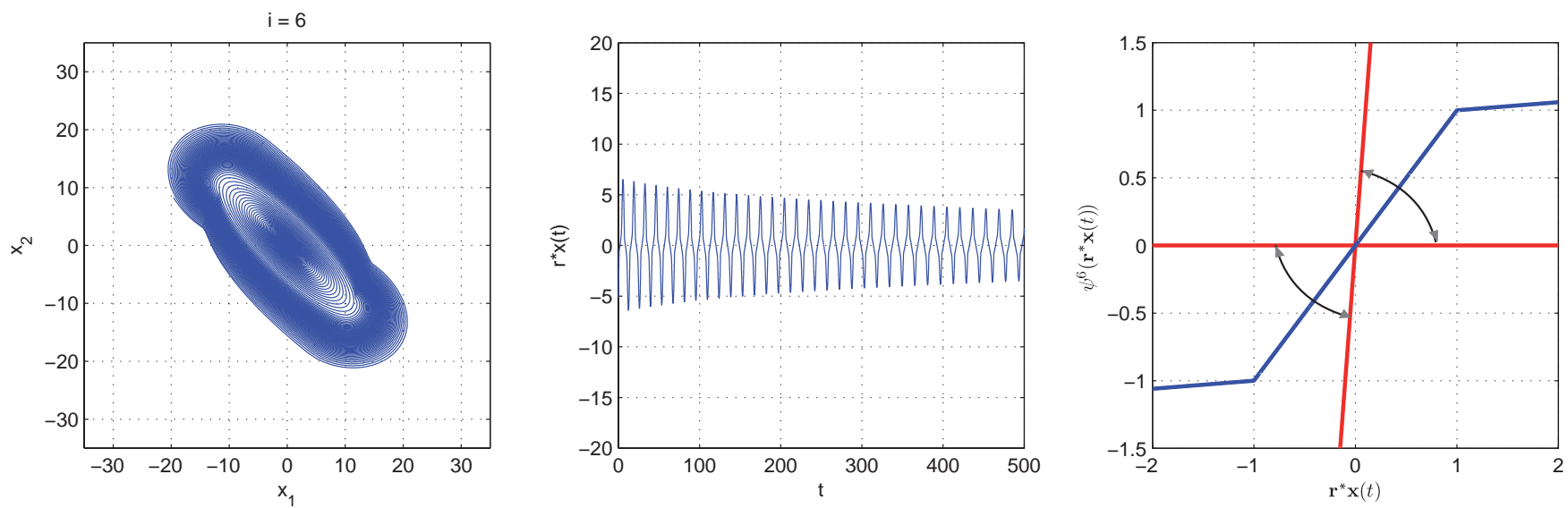

Fig. 50. A projection of trajectory on the plane $\left(x_{1}, x_{2}\right)$ and the output of system for $i=6$; the linear stability sector and the nonlinearity $\psi^{6}(\sigma)$. 

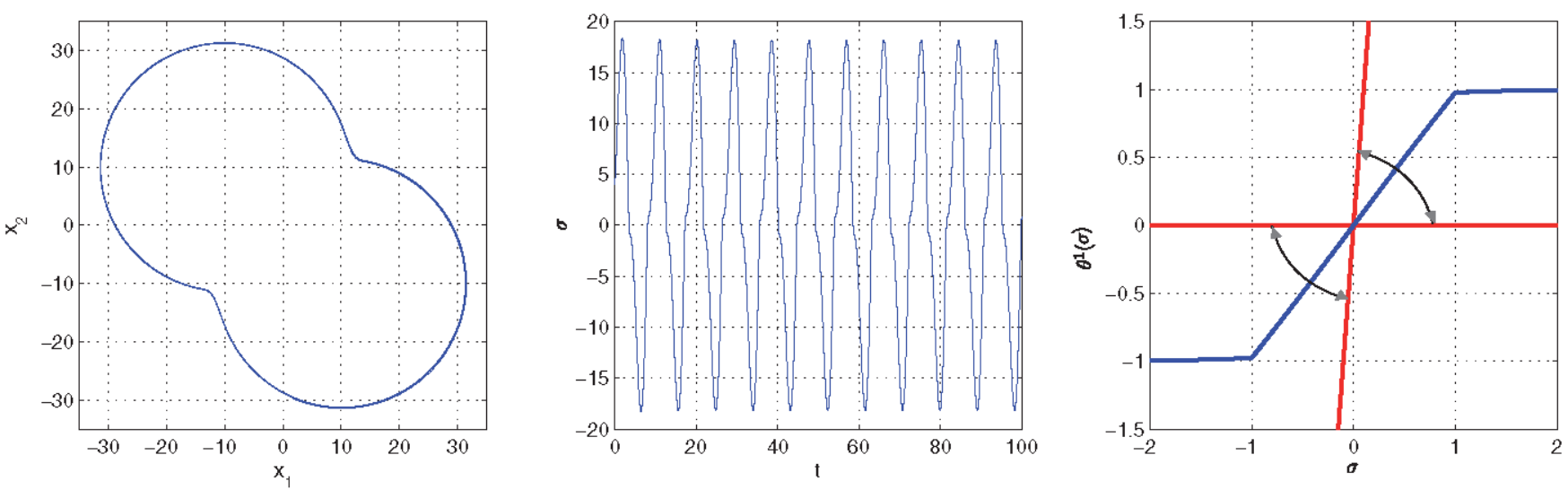

Fig. 51. A projection of trajectory on the plane $\left(x_{1}, x_{2}\right)$ and the output of system for $i=1$; the linear stability sector and the nonlinearity $\theta^{1}(\sigma)$.

where $i=1, \ldots, 10, m=10$,

$\tanh (\sigma)=\frac{e^{\sigma}-e^{-\sigma}}{e^{\sigma}+e^{-\sigma}}, \quad 0<\frac{d}{d \sigma} \tanh (\sigma) \leq 1 \quad \forall \sigma$.

For $i=1$, starting from the point $\mathbf{x}^{10}(T)$, one can find a periodic solution and continue the procedure of computing periodic solutions for $i=$ $2, \ldots, 10$. The result of the algorithm operation is shown in Figs. 51-53.

Thus, it can be obtained a periodic solution for a system with the smooth monotonically increasing nonlinearity $\theta^{10}(\sigma)=\tanh (\sigma)$, satisfying Kalman's condition.

Compare the described algorithm with investigations, devoted to Kalman's problem, and show its application to the construction of counterexamples.

Consider the only widely cited in literature (see, e.g. [Blondel \& Megretski, 2004; Hsu \& Meyer, 1968; Westphal, 2001]) Fitts' counterexample [Fitts,
1966]. In [Fitts, 1966], a simulation of system (98) is given for $n=4$ with the transfer function

$$
W(p)=\frac{p^{2}}{\left[(p+\beta)^{2}+(0.9)^{2}\right]\left[(p+\beta)^{2}+(1.1)^{2}\right]}
$$

and with cubic nonlinearity $\varphi(\sigma)=k \sigma^{3}$ for certain parameters $\beta$ and $k$. Subsequently, for a part of parameters $\beta \in(0.572,0.75)$, considered by Fitts, it was shown [Barabanov, 1982, 1988] that the results of Fitts' experiments are not valid. The authors of the present paper have made computer simulations of the considered above system and for values of parameters $\beta=0.01$ and $k=10$, a "periodic" solution with very small domain of attraction was obtained. The smallness of this domain makes difficult the use of the algorithm suggested in the present paper. Consider simulation of Fitts' system for $\beta=0.01$ and $k=10$. Restoring the system by
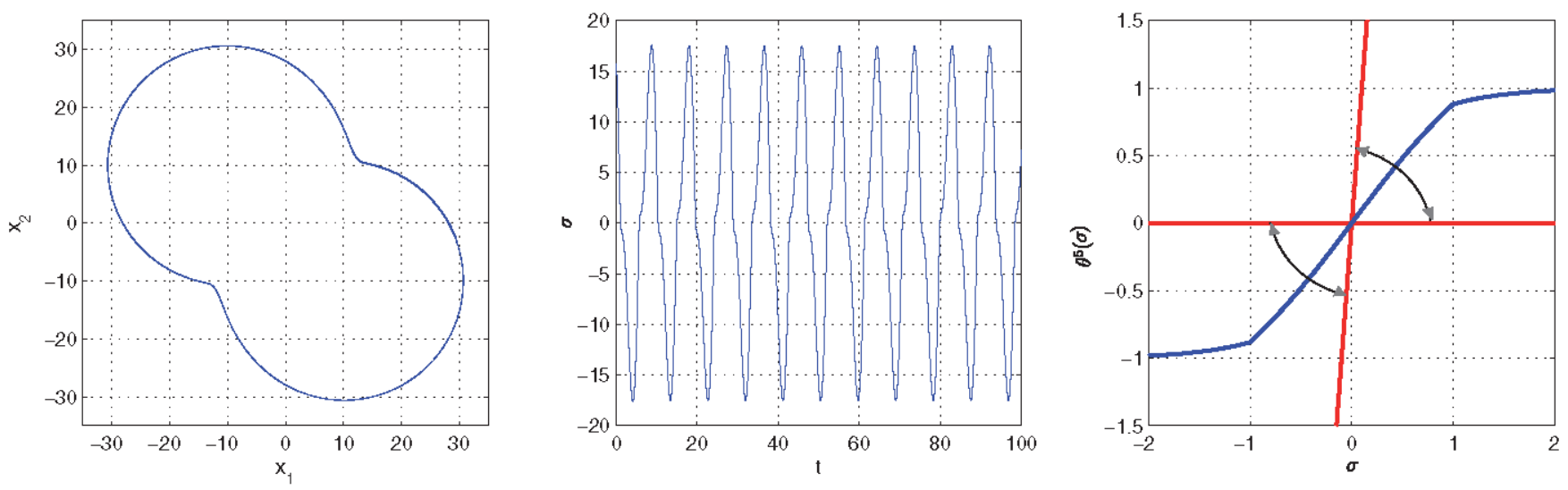

Fig. 52. A projection of trajectory on the plane $\left(x_{1}, x_{2}\right)$ and the output of system for $i=5$; the linear stability sector and the nonlinearity $\theta^{5}(\sigma)$. 

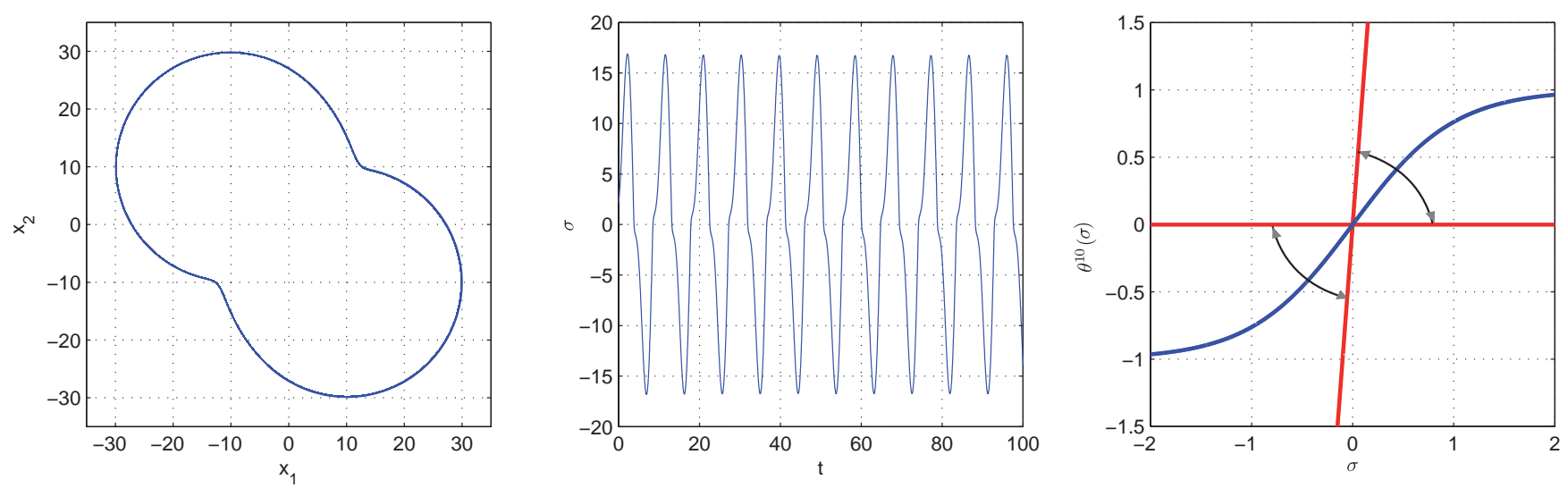

Fig. 53. A projection of trajectory on the plane $\left(x_{1}, x_{2}\right)$ and the output of system for $i=10$; the linear stability sector and the nonlinearity $\tanh (\sigma)=\theta^{10}(\sigma)$.

transfer function (163), one can obtain

$$
\begin{aligned}
\dot{x}_{1}= & x_{2}, \\
\dot{x}_{2}= & x_{3}, \\
\dot{x}_{3}= & x_{4}, \\
\dot{x}_{4}= & -0.9803 x_{1}-0.0404 x_{2}-2.0206 x_{3} \\
& \quad-0.0400 x_{4}+\varphi\left(-x_{3}\right), \quad \varphi(\sigma)=10 \sigma^{3} .
\end{aligned}
$$

By simulation of this system with the initial data $x_{1}(0)=85.1189, \quad x_{2}(0)=0.9222, \quad x_{3}(0)=$ $-2.0577, x_{4}(0)=-2.6850$, a "periodic" solution (Fig. 54) can be defined. These initial data were obtained empirically after the authors' long search in four-dimensional phase space.

Let us proceed to the system, considered by Barabanov [1982, 1988]. As in [Barabanov, 1988], the proof of existence of system (98) for $n=4$, for which Kalman's problem has negative solution, is, in essence, the "existence theorem" and, therefore, it needs to be carefully checked.

Consider a system studied in [Barabanov, 1988]

$$
\begin{aligned}
& \dot{x}_{1}=x_{2}, \\
& \dot{x}_{2}=-x_{4}, \\
& \dot{x}_{3}=x_{1}-2 x_{4}-\varphi\left(x_{4}\right), \quad \varphi(\sigma)=\operatorname{sign}(\sigma) . \\
& \dot{x}_{4}=x_{1}+x_{3}-x_{4}-\varphi\left(x_{4}\right),
\end{aligned}
$$

Here a sector of linear stability is $(0,+\infty)$ and, according to [Barabanov, 1988], this system has a periodic solution, which survives under small perturbation of nonlinearity (i.e. for construction of a counterexample to Kalman's conjecture, it is necessary somehow to choose small perturbation of $\varphi(\sigma)$ in order to obtain a strictly increasing function). On the existence of periodic solutions in system (165) Bernat and Llibre [1996] wrote “...we checked numerically that in the region where he
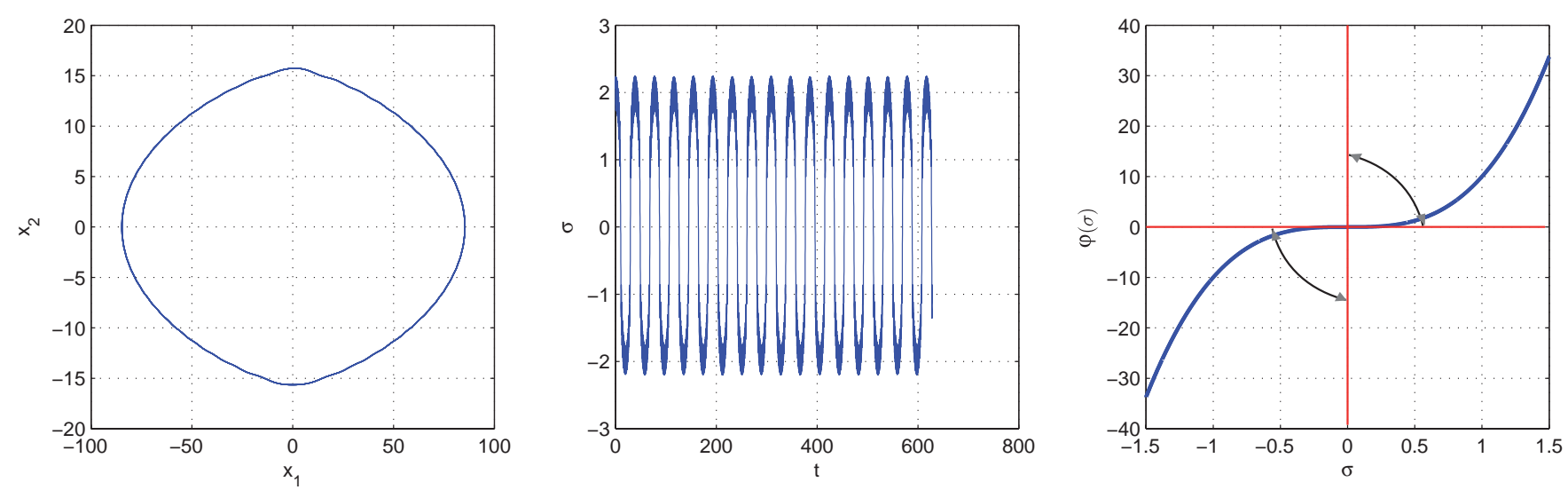

Fig. 54. A projection of trajectory on the plane $\left(x_{1}, x_{2}\right)$, the output of system $\sigma(t)$, the graph of $\varphi(\sigma)$. 
[Barabanov] tries to find the periodic orbit all solutions have $\omega$-limit equal to the origin".

We should pay attention here to the difficulties of simulation of systems with the nonlinearity $\operatorname{sign}(\sigma)$.

Consider, for example, a classical differential equation

$$
\dddot{x}+A \ddot{x}+B \dot{x}+x=-\operatorname{sign}(\ddot{x}),
$$

describing the operation of Watt's regulator under dry friction [Andronov \& Maier, 1947; Yakobovich et al., 2004]. Having made in Eq. (166) the change $y_{1}=\ddot{x}, y_{2}=-B \dot{x}-x, y_{3}=-\dot{x}$, one can obtain the following system

$$
\begin{aligned}
& \dot{y}_{1}=-A y_{1}+y_{2}-\operatorname{sign}\left(y_{1}\right), \\
& \dot{y}_{2}=-B y_{1}+y_{3}, \\
& \dot{y}_{3}=-y_{1} .
\end{aligned}
$$

It is well known [Yakobovich et al., 2004], that in this system the remaining segment $y_{1}=0, y_{3}=0$, $y_{2} \in[-1,1]$ is stable in the large for $A B>1$.

Suppose that $A=1.5, B=1.1$, and carry out a trajectories' simulation of system (167) with the initial data, $y_{1}(0)=-0.5, y_{2}(0)=1$, $y_{3}(0)=1.2$

Figure 55 shows that the computation of trajectories in the neighborhood of segment of rest after a transient process (Fig. 55, on the left) gives small oscillations, arising due to the errors of computation of trajectories and the discontinuity of nonlinearity $\operatorname{sign}(\cdot)$ (Fig. 55, on the right). On the other hand, if in system $(167)$, in changing $\operatorname{sign}(\cdot)$ to the continuous in zero nonlinearity sat $(\cdot)$, a similar numerical procedure "converges" to zero (Fig. 56).

Let us apply our algorithm to system (165) considered by Barabanov, having changed the form of nonlinearity to

$$
\varphi(\sigma)= \begin{cases}5 \sigma, & \forall|\sigma| \leq \frac{1}{5} \\ \operatorname{sign}(\sigma) & \forall|\sigma|>\frac{1}{5} .\end{cases}
$$

Reduce the system to form (157) by the transformation matrix $\mathbf{S}$, and compute $\mathbf{x}(0)$ using (160). Then at the first step of algorithm for system (165) with $\varphi(\sigma)$ of the form (168) one obtains the initial data $\mathbf{S x}(0)=(0,0.2309,0.2309,0)$. The results of algorithm operation are shown in Figs. 57-59.

Continue sequential construction of periodic solutions, make change nonlinearity (168) to a strictly increasing function $\psi^{i}(\sigma)(161)$, where $\mu=$ $1, N=0.01$. The obtained periodic solution for $i=3$ is shown in Fig. 60 .

Thus, a counterexample to Kalman's conjecture is constructed in a modified Barabanov's system.
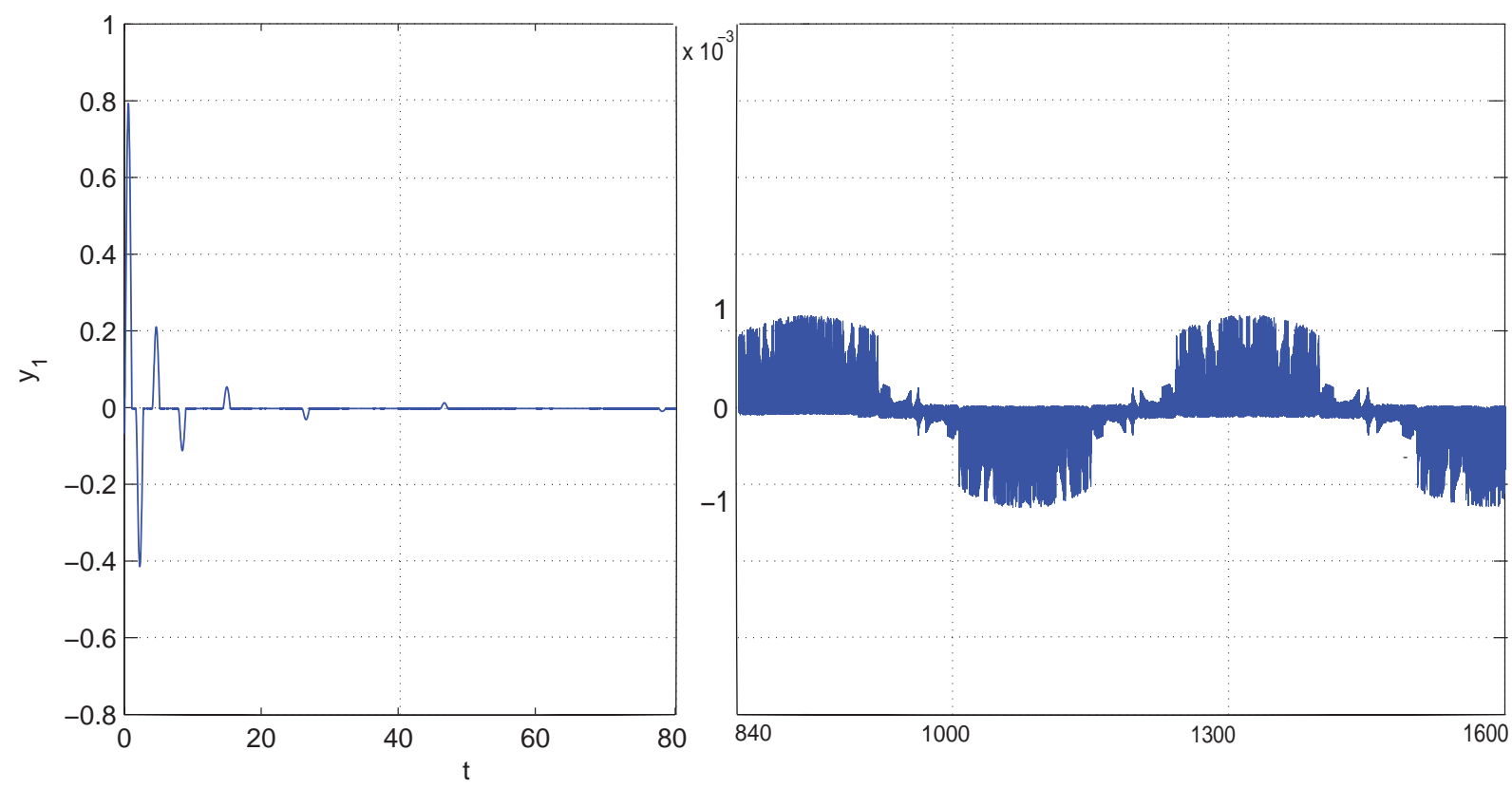

Fig. 55. The output $y_{1}$ of system with the nonlinearity $\operatorname{sign}\left(y_{1}\right)$. 

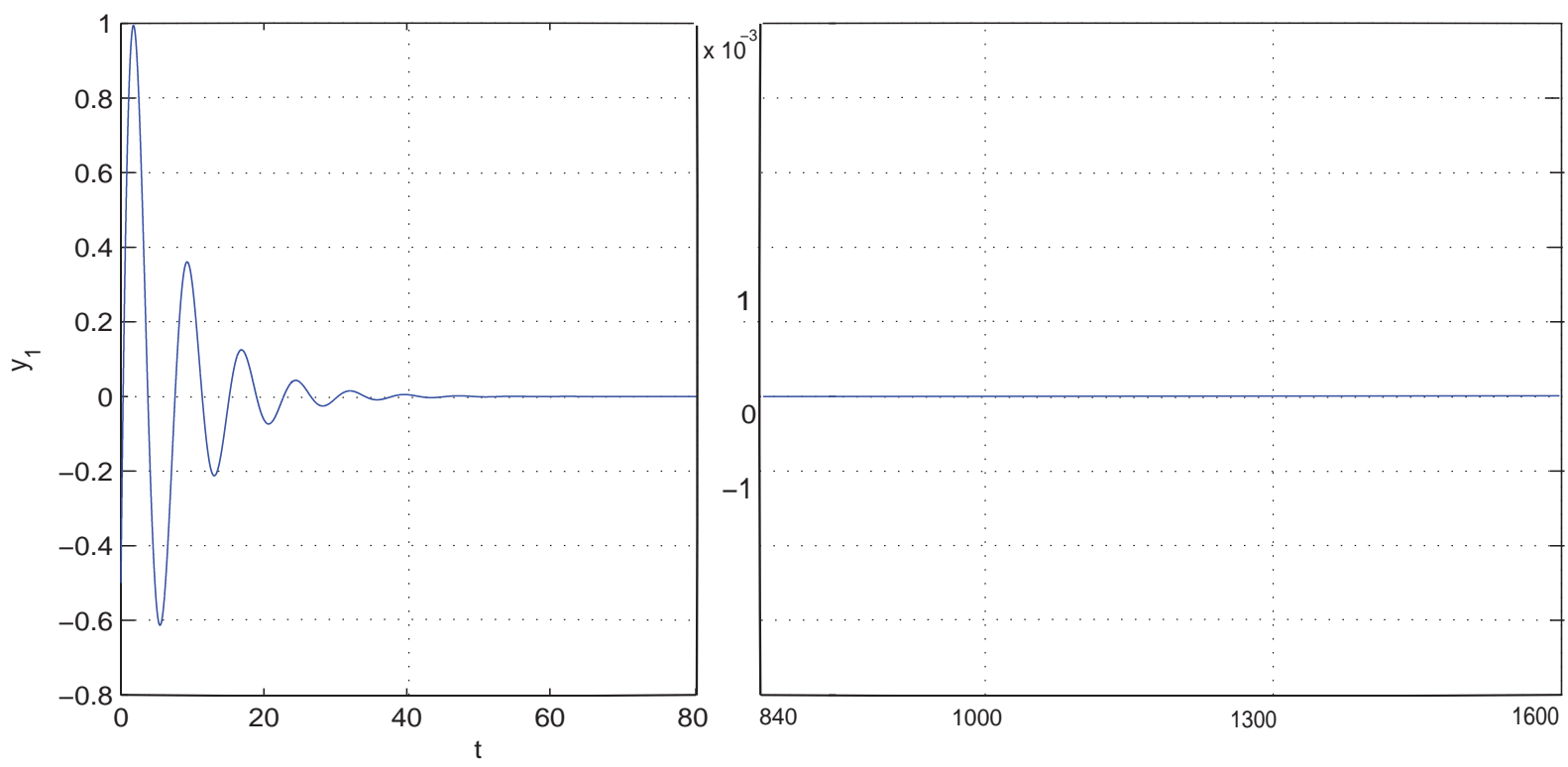

Fig. 56. The output $y_{1}$ of system with the nonlinearity sat $\left(y_{1}\right)$.
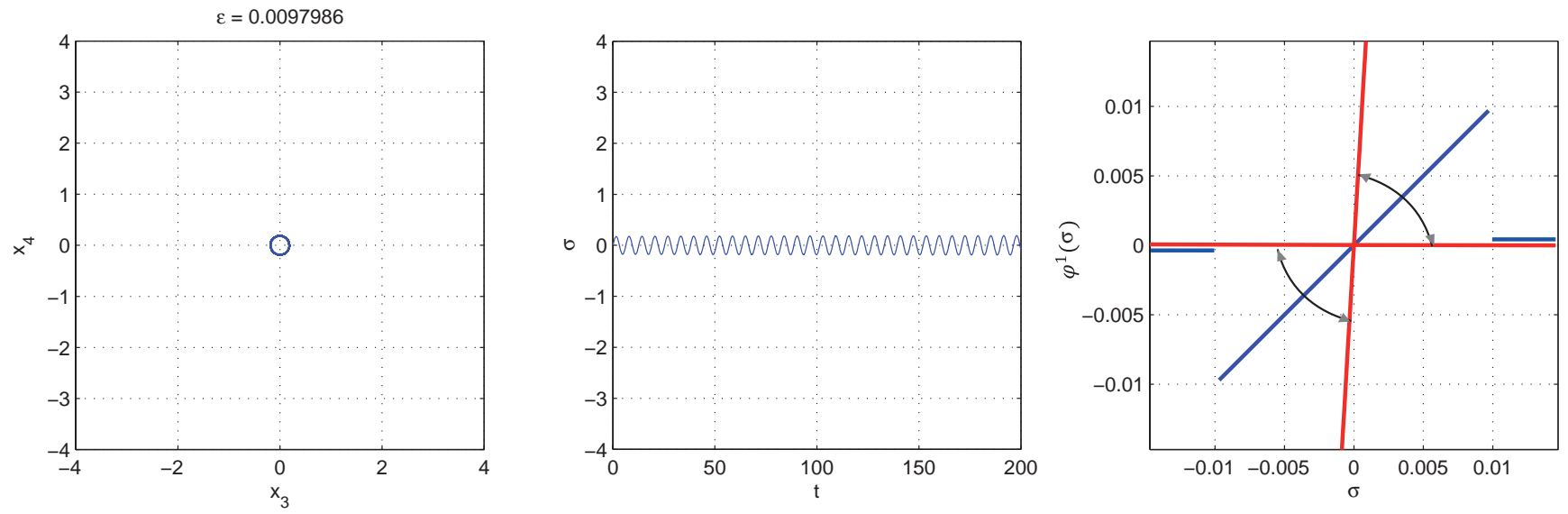

Fig. 57. A projection of trajectory on the plane $\left(x_{3}, x_{4}\right)$; the output of system; the nonlinearity and the linear stability sector; $j=1$.
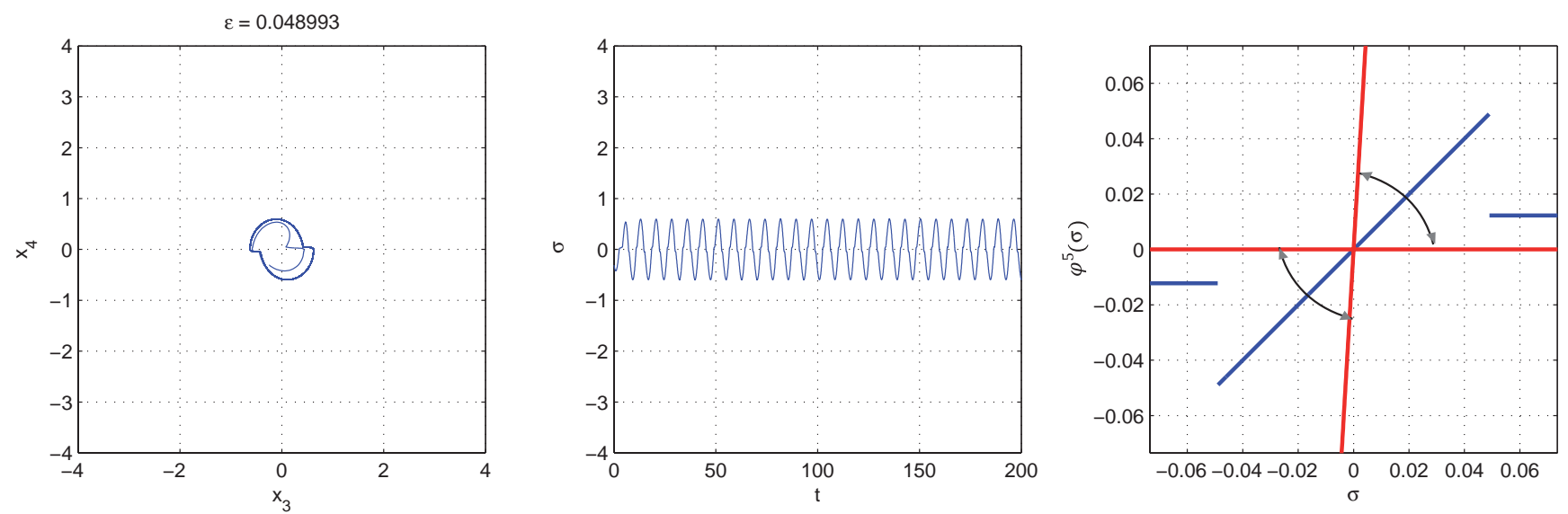

Fig. 58. A projection of trajectory on the plane $\left(x_{3}, x_{4}\right)$; the output of system; the nonlinearity and the linear stability sector; $j=5$. 

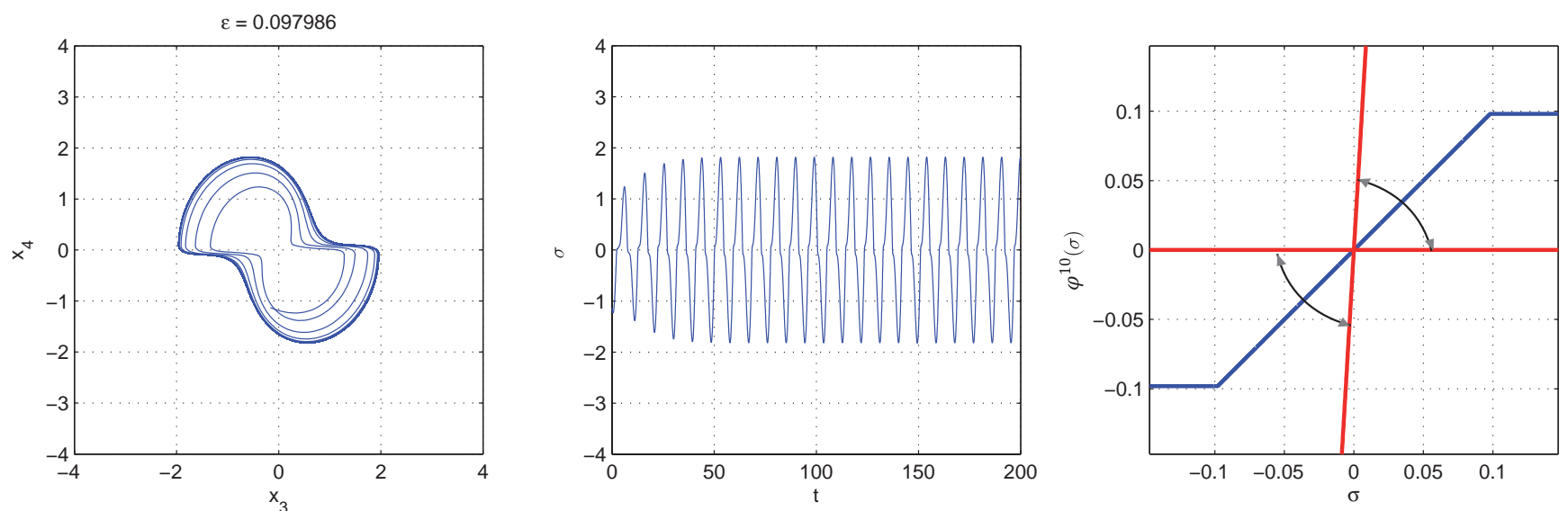

Fig. 59. A projection of trajectory on the plane $\left(x_{3}, x_{4}\right)$; the output of system; the nonlinearity $\varphi(\sigma)$ and the linear stability sector; $j=10$.

Proceed now to Bernat and Llibre's investigations [Bernat \& Llibre, 1996; Meisters, 1996; Glutsyuk, 1998], there were revealed some "gaps" in the work [Barabanov, 1988]: thus, in [Bernat \& Llibre, 1996] it is written "He [Barabanov] tried to prove that this system and systems close to this have a periodic orbit. But his arguments are not complete", in [Meisters, 1996] it can be found the following remark "In 1988, Barabanov gave ideas for constructing a class $C^{1}$ MY-SYSTEM [Markus-Yamabe system] in four dimensions with a nonconstant periodic orbit - and hence a counterexample to MYC [Markus-Yamabe Conjecture] in $\mathbb{R}^{4}$. But the details of his paper were in some doubt", in [Glutsyuk, 1998] one can read "In 1988, Barabanov made an attempt to construct a counterexample to this theorem in $\mathbb{R}^{n}$ for $n \geq$ 4. Errors in his paper were found recently." In [Bernat \& Llibre, 1996] an attempt was made to overcome these "gaps" by analytical-numerical methods, based on Andronov's point transformation method [Andronov et al., 1966].

Now our algorithm will be applied to the system proposed in [Bernat \& Llibre, 1996],

$$
\begin{aligned}
& \dot{x}_{1}=x_{2}, \\
& \dot{x}_{2}=-x_{4}, \\
& \dot{x}_{3}=x_{1}-2 x_{4}-\frac{9131}{900} \varphi\left(x_{4}\right), \\
& \dot{x}_{4}=x_{1}+x_{3}-x_{4}-\frac{1837}{180} \varphi\left(x_{4}\right),
\end{aligned}
$$

where

$$
\varphi(\sigma)= \begin{cases}\sigma, & \forall|\sigma| \leq \frac{900}{9185} \\ \operatorname{sign}(\sigma) \frac{900}{9185}, & \forall|\sigma|>\frac{900}{9185}\end{cases}
$$
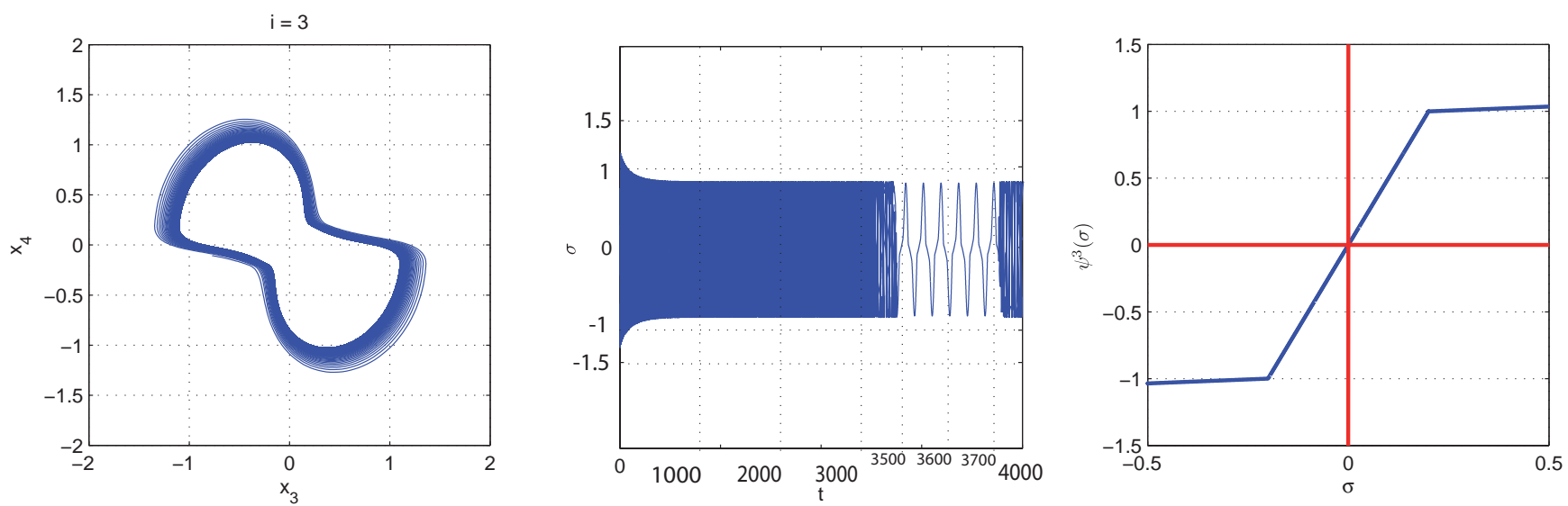

Fig. 60. A projection of trajectory on the plane $\left(x_{3}, x_{4}\right)$; the nonlinearity and the linear stability sector; $i=3$. 

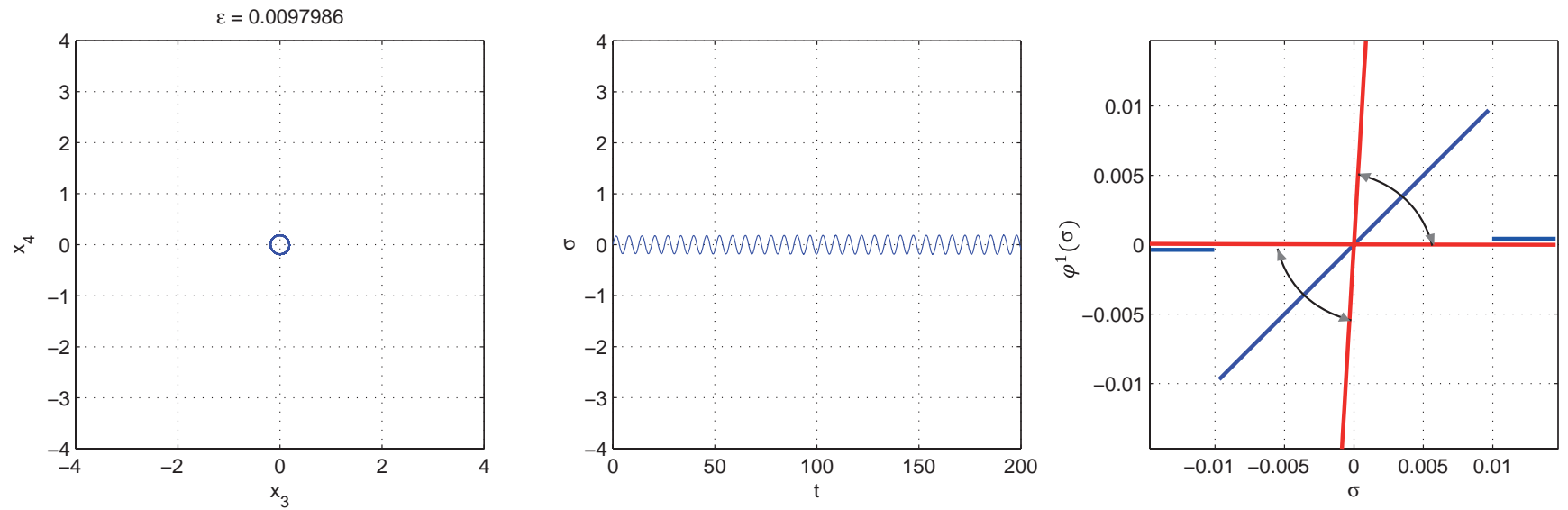

Fig. 61. A projection of trajectory on the plane $\left(x_{3}, x_{4}\right)$; the output of system; a nonlinearity and stability sector; $j=1$.
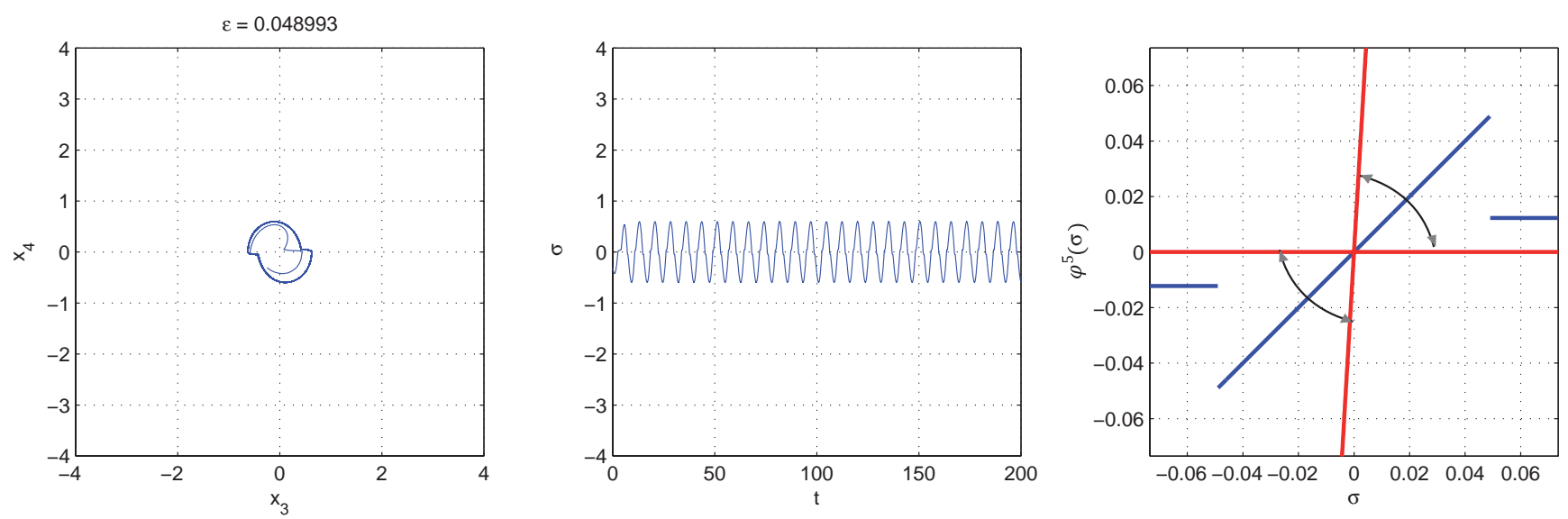

Fig. 62. A projection of trajectory on the plane $\left(x_{3}, x_{4}\right)$; the output of system; a nonlinearity and stability sector; $j=5$.
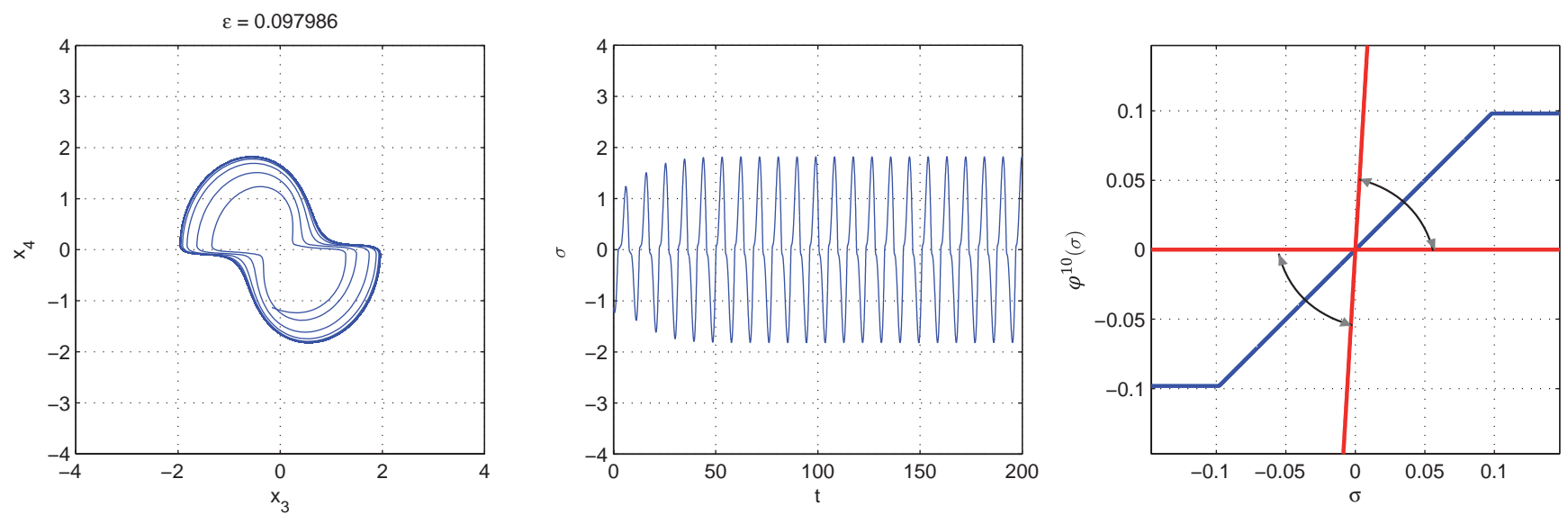

Fig. 63. A projection of trajectory on the plane $\left(x_{3}, x_{4}\right)$; the output of system; a nonlinearity and stability sector; $j=10$. 

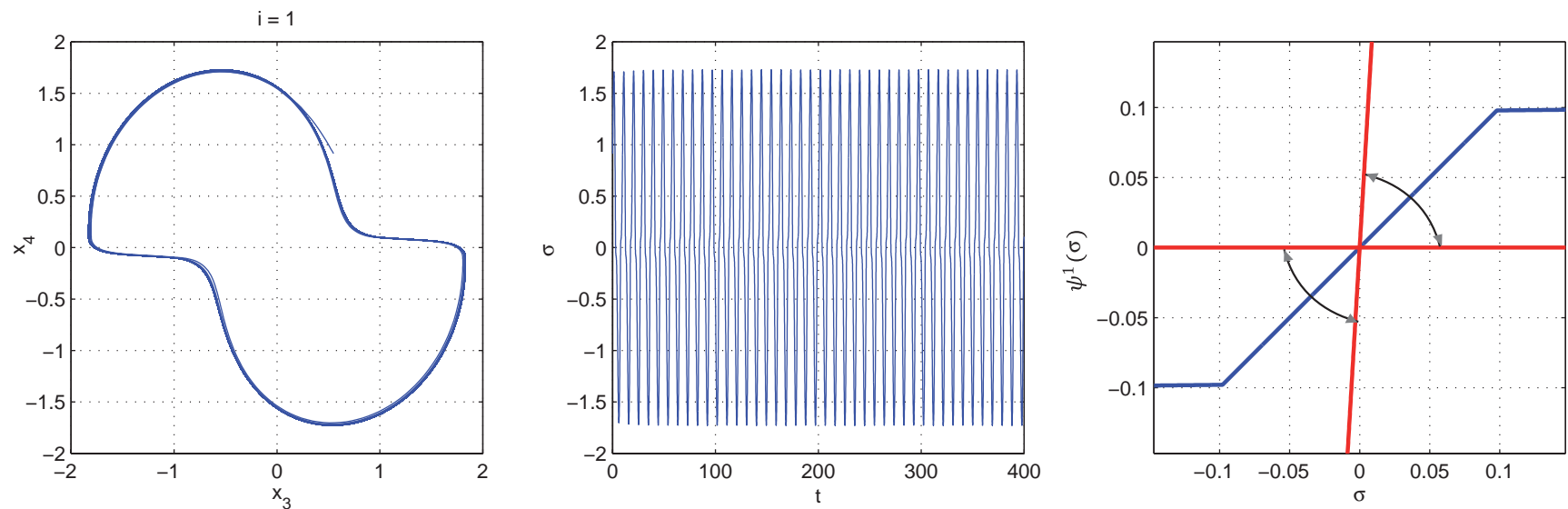

Fig. 64. A projection of trajectory on the plane $\left(x_{3}, x_{4}\right)$; the output of system; a nonlinearity and stability sector; $i=1$.
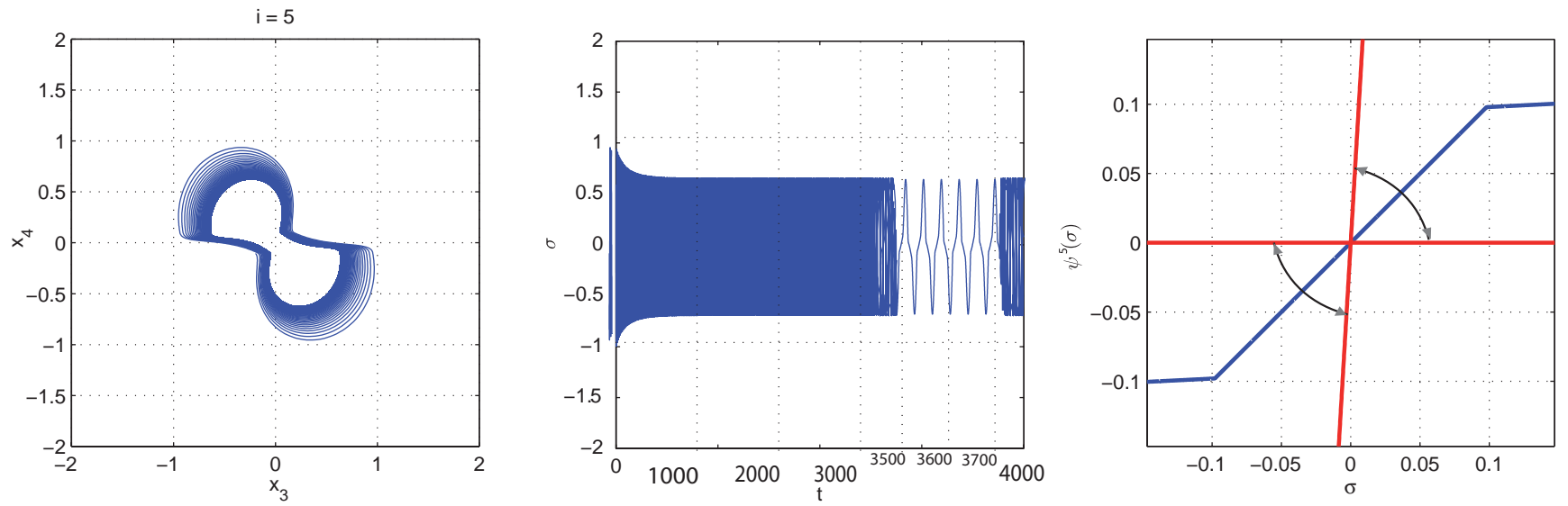

Fig. 65. A projection of trajectory on the plane $\left(x_{3}, x_{4}\right)$; the output of system; a nonlinearity and stability sector; $i=5$.
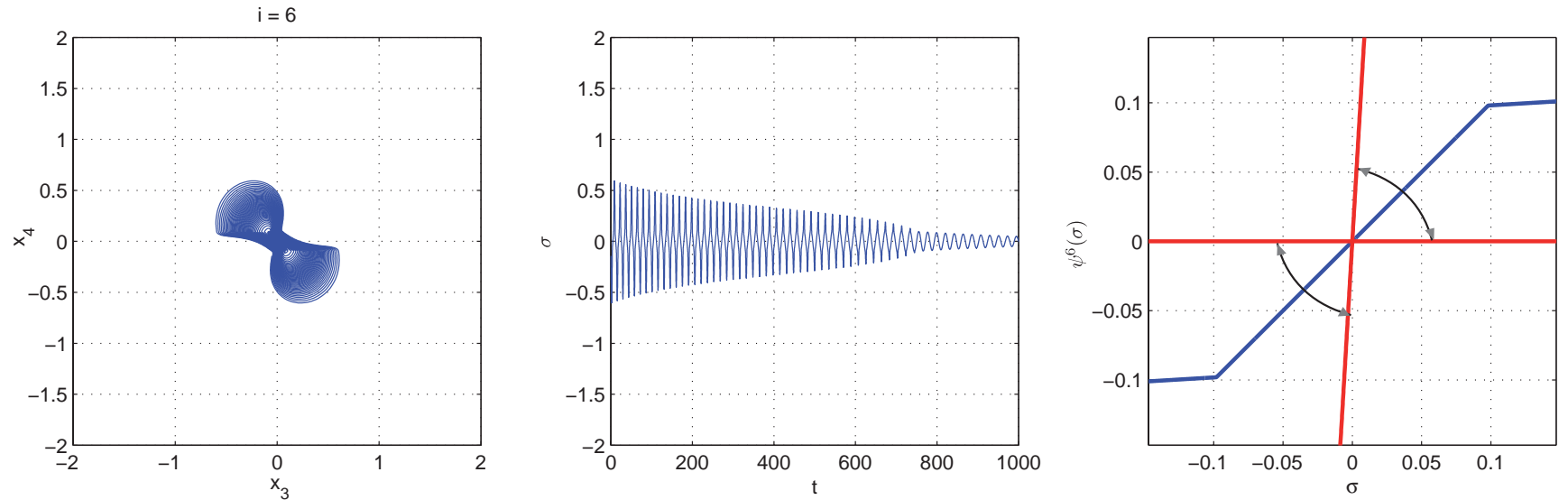

Fig. 66. A projection of trajectory on the plane $\left(x_{3}, x_{4}\right)$; the output of system; a nonlinearity and stability sector; $i=6$. 
Reducing the system to the form (157) by the transformation matrix $\mathbf{S}$ and computing $\mathbf{x}(0)$ by (160), for system (169) one can obtain at the first step of algorithm, the initial data $\mathbf{S x}(0)=$ $(0,0.1722,0.1722,0)$.

The result of operation of the algorithm, suggested above, is shown in Figs. 61-63.

Here the nonlinearity $\varphi(\sigma)$ is changed to the strictly increasing function $\psi^{i}(\sigma)$ (161) with $\mu=$ $1, \varepsilon_{m}=1, N=0.01$, for $i=1, \ldots, 6$. The periodic solutions obtained are shown in Figs. 64 and 65.

In computing a solution for $i=6$, a periodic solution (Fig. 66) vanishes.

Note that the second part of the algorithm, where the saturation zone of the nonlinearities considered arise and counterexamples to Kalman's problem are constructed, complements the numerical results from [Barabanov, 1988; Bernat \& Llibre, 1996], where the nonlinearities $\operatorname{sign}(\sigma)$ and $\operatorname{sat}(\sigma)$ are considered, that do not satisfy Kalman's conditions. Thus, here we see that the algorithm suggested can be used for the case of Barabanov's and Bernat's and Llibre's modified systems.

\section{Hidden Attractor in Chua's Circuits}

The development of modern computers allows one to perform the numerical simulation of nonlinear chaotic systems, and to obtain new information on the structure of their trajectories. Classical attractors in well-known chaotic dynamical systems of Lorenz [1963], Chua [Chua \& Lin, 1990], Chen [Chen \& Ueta, 1999], and many others are selfexcited attractors and can be obtained numerically by the standard computational procedure. However, there are chaotic attractors of another type: hidden chaotic attractors, for which the possibility of such simple computational approach turn out to be highly limited. In 2010, for the first time, a hidden chaotic attractor was discovered [Kuznetsov et al., 2010; Bragin et al., 2011; Kuznetsov et al., 2011a; Leonov et al., 2011c, 2012a] in Chua's circuit, described by three-dimensional dynamical system.

Note that Chua himself, in analyzing various cases of attractors' existence in Chua's circuit [Chua \& Lin, 1990] did not admit the existence of hidden attractor (discovered later) in the circuit.

Below we will demonstrate the application of the above algorithm to the localization of hidden chaotic attractor in Chua's system. For this purpose, write Chua's system in the form (98)

$$
\frac{d \mathbf{x}}{d t}=\mathbf{P} \mathbf{x}+\mathbf{q} \psi\left(\mathbf{r}^{*} \mathbf{x}\right), \quad \mathbf{x} \in \mathbb{R}^{3} .
$$

Here

$$
\begin{gathered}
\mathbf{P}=\left(\begin{array}{ccc}
-\alpha\left(m_{1}+1\right) & \alpha & 0 \\
1 & -1 & 1 \\
0 & -\beta & -\gamma
\end{array}\right), \\
\mathbf{q}=\left(\begin{array}{c}
-\alpha \\
0 \\
0
\end{array}\right), \quad \mathbf{r}=\left(\begin{array}{l}
1 \\
0 \\
0
\end{array}\right), \\
\psi(\sigma)=\left(m_{0}-m_{1}\right) \operatorname{sat}(\sigma) .
\end{gathered}
$$

Introduce a coefficient $k$ and a small parameter $\varepsilon$, and represent system (171) as (103)

$$
\frac{d \mathbf{x}}{d t}=\mathbf{P}_{\mathbf{0}} \mathbf{x}+\mathbf{q} \varepsilon \varphi\left(\mathbf{r}^{*} \mathbf{x}\right),
$$

where

$$
\begin{aligned}
\mathbf{P}_{\mathbf{0}} & =\mathbf{P}+k \mathbf{q r}^{*} \\
& =\left(\begin{array}{ccc}
-\alpha\left(m_{1}+1+k\right) & \alpha & 0 \\
1 & -1 & 1 \\
0 & -\beta & -\gamma
\end{array}\right), \\
\lambda_{1,2}^{\mathbf{P}_{0}} & = \pm i \omega_{0}, \quad \lambda_{3}^{\mathbf{P}_{0}}=-d, \\
\varphi(\sigma) & =\psi(\sigma)-k \sigma=\left(m_{0}-m_{1}\right) \operatorname{sat}(\sigma)-k \sigma .
\end{aligned}
$$

By the nonsingular linear transformation $\mathbf{x}=\mathbf{S y}$, system (172) is reduced to the form (106)

$$
\frac{d \mathbf{y}}{d t}=\mathbf{A y}+\mathbf{b} \varepsilon \varphi\left(\mathbf{c}^{*} \mathbf{y}\right)
$$

where

$$
\begin{gathered}
\mathbf{A}=\left(\begin{array}{ccc}
0 & -\omega_{0} & 0 \\
\omega_{0} & 0 & 0 \\
0 & 0 & -d
\end{array}\right), \\
\mathbf{b}=\left(\begin{array}{c}
b_{1} \\
b_{2} \\
1
\end{array}\right), \quad \mathbf{c}=\left(\begin{array}{c}
1 \\
0 \\
-h
\end{array}\right) .
\end{gathered}
$$

The transfer function $W_{\mathbf{A}}(p)$ of system (173) can be represented as

$$
W_{\mathbf{A}}(p)=\frac{-b_{1} p+b_{2} \omega_{0}}{p^{2}+\omega_{0}^{2}}+\frac{h}{p+d} .
$$


Further, using the equality of transfer functions of systems (172) and (173), one can obtain

$$
W_{\mathbf{A}}(p)=\mathbf{r}^{*}\left(\mathbf{P}_{0}-p \mathbf{I}\right)^{-1} \mathbf{q} .
$$

This implies the following relations

$$
\begin{aligned}
& k=\frac{-\alpha\left(m_{1}+m_{1} \gamma+\gamma\right)+\omega_{0}^{2}-\gamma-\beta}{\alpha(1+\gamma)}, \\
& d=\frac{\alpha+\omega_{0}^{2}-\beta+1+\gamma+\gamma^{2}}{1+\gamma} \\
& h=\frac{\alpha\left(\gamma+\beta-(1+\gamma) d+d^{2}\right)}{\omega_{0}^{2}+d^{2}} \\
& b_{1}=\frac{\alpha\left(\gamma+\beta-\omega_{0}^{2}-(1+\gamma) d\right)}{\omega_{0}^{2}+d^{2}} \\
& b_{2}=\frac{\alpha\left((1+\gamma-d) \omega_{0}^{2}+(\gamma+\beta) d\right)}{\omega_{0}\left(\omega_{0}^{2}+d^{2}\right)} .
\end{aligned}
$$

Since by the nonsingular linear transformation $\mathbf{x}=$ Sy system (172) can be reduced to the form (173), for the matrix $\mathbf{S}$ the following relations

$$
\mathbf{A}=\mathbf{S}^{-1} \mathbf{P}_{0} \mathbf{S}, \quad \mathbf{b}=\mathbf{S}^{-1} \mathbf{q}, \quad \mathbf{c}^{*}=\mathbf{r}^{*} \mathbf{S}
$$

are valid. Having solved these matrix equations, one can obtain the transformation matrix

$$
\mathbf{S}=\left(\begin{array}{lll}
s_{11} & s_{12} & s_{13} \\
s_{21} & s_{22} & s_{23} \\
s_{31} & s_{32} & s_{33}
\end{array}\right)
$$

where

$$
\begin{aligned}
& s_{11}=1, \quad s_{12}=0, \quad s_{13}=-h, \\
& s_{21}=m_{1}+1+k, \quad s_{22}=-\frac{\omega_{0}}{\alpha}, \\
& s_{23}=-\frac{h\left(\alpha\left(m_{1}+1+k\right)-d\right)}{\alpha}, \\
& s_{31}=\frac{\alpha\left(m_{1}+k\right)-\omega_{0}^{2}}{\alpha}, \\
& s_{32}=-\frac{\alpha(\beta+\gamma)\left(m_{1}+k\right)+\alpha \beta-\gamma \omega_{0}^{2}}{\alpha \omega_{0}}, \\
& s_{33}=h \frac{\alpha\left(m_{1}+k\right)(d-1)+d(1+\alpha-d)}{\alpha} .
\end{aligned}
$$

By (119), for sufficiently small $\varepsilon$, at the first step of multistage localization procedure, one obtains the initial data

$$
\mathbf{x}(0)=\mathbf{S y}(0)=\mathbf{S}\left(\begin{array}{c}
a_{0} \\
0 \\
0
\end{array}\right)=\left(\begin{array}{l}
a_{0} s_{11} \\
a_{0} s_{21} \\
a_{0} s_{31}
\end{array}\right) .
$$

Returning to Chua's system denotations, for the determination of initial data of starting solution for multistage procedure, one can obtain

$$
\begin{gathered}
x(0)=a_{0}, \quad y(0)=a_{0}\left(m_{1}+1+k\right), \\
z(0)=a_{0} \frac{\alpha\left(m_{1}+k\right)-\omega_{0}^{2}}{\alpha} .
\end{gathered}
$$

Consider system (172) with the parameters

$$
\begin{gathered}
\alpha=8.4562, \quad \beta=12.0732, \quad \gamma=0.0052, \\
m_{0}=-0.1768, \quad m_{1}=-1.1468 .
\end{gathered}
$$

Note that for the considered values of parameters there are three equilibria in the system: a locally stable zero equilibrium and two saddle equilibria. Now let us apply the above procedure of hidden attractors localization to Chua's system (171) with parameters (177). For this purpose, compute a starting frequency and a coefficient of harmonic linearization:

$$
\omega_{0}=2.0392, \quad k=0.2098 .
$$

Then, we compute solutions of system (172) with the nonlinearity $\varepsilon \varphi(x)=\varepsilon(\psi(x)-k x)$, sequentially increasing $\varepsilon$ from the value $\varepsilon_{1}=0.1$ to $\varepsilon_{10}=1$ with step 0.1. By (174) and (176), the initial data can be obtained

$x(0)=9.4287, \quad y(0)=0.5945, \quad z(0)=-13.4705$

for the first step of multistage procedure. For $\varepsilon_{1}=$ 0.1 , after a transient process, the computational procedure reaches starting oscillation $\mathbf{x}^{1}(t)$. Further, by the numerical procedure and the sequential transformation $\mathbf{x}^{j}(t)$ with increasing parameter $\varepsilon_{j}$, for original Chua's system (171) the set $\mathcal{A}_{\text {hidden }}$ is computed. This set is shown in Fig. 67.

It should be noted that the decreasing of integration step, the increasing of integration time, and the computation of different trajectories of the original system with initial data from a small neighborhood of $\mathcal{A}_{\text {hidden }}$ lead to the localization of the same set $\mathcal{A}_{\text {hidden }}$ (all computed trajectories densely trace the set $\left.\mathcal{A}_{\text {hidden }}\right)$. Note also that for the computed trajectories, we observe 

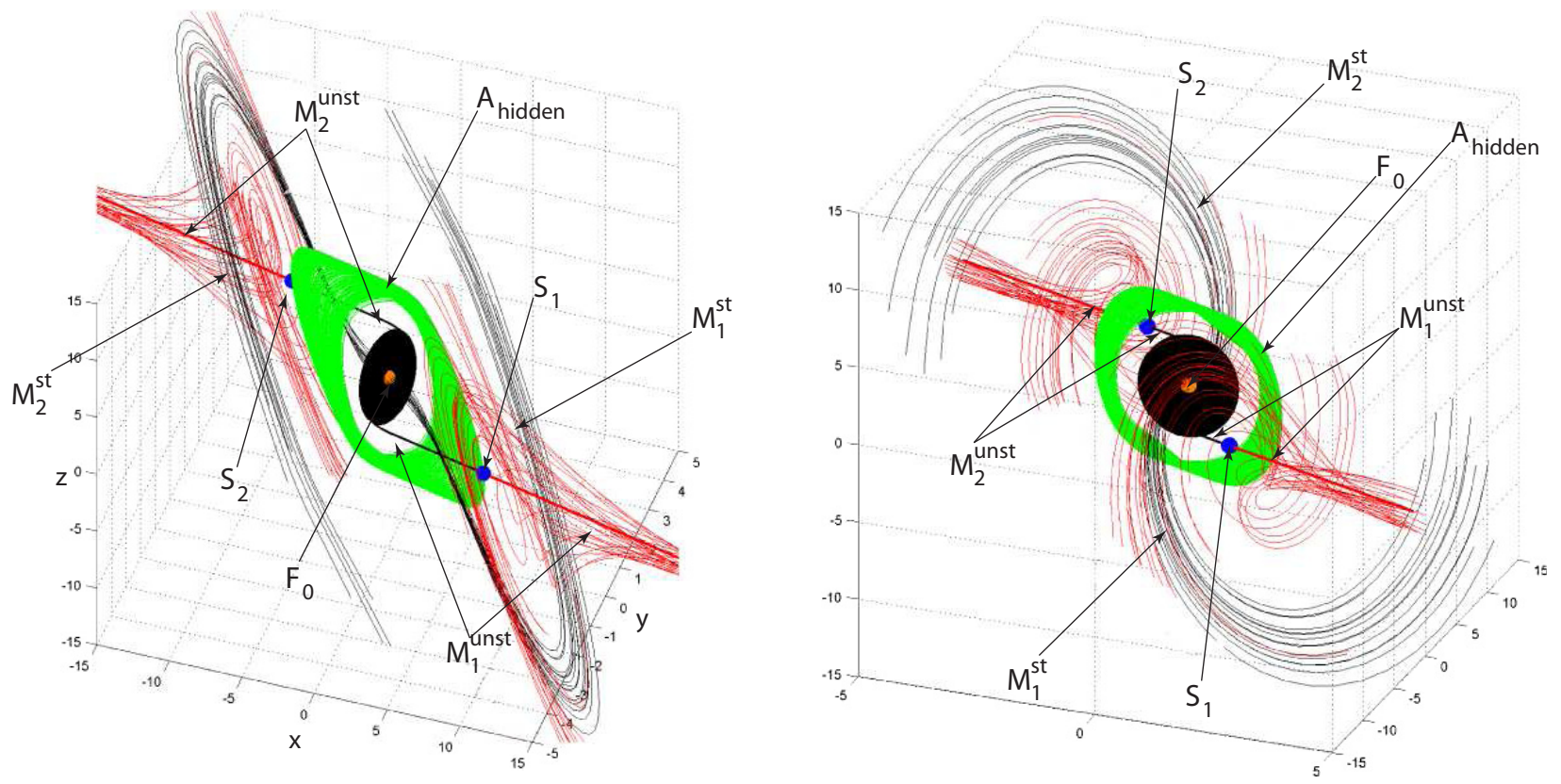

Fig. 67. Equilibria, saddles manifolds, hidden attractor localization.

Zhukovsky instability and the positiveness of Lyapunov exponent [Leonov \& Kuznetsov, 2007b]. ${ }^{3}$

The behavior of system trajectories in a neighborhood of equilibria is shown in Fig. 67. Here $M_{1,2}^{\text {unst }}$ are unstable manifolds, $M_{1,2}^{\text {st }}$ are stable manifolds. Thus, in phase space of system, there are stable separating manifolds of saddles.

The above results together with the remark on the existence, in system, of locally stable zero equilibrium $F_{0}$, attracting the stable manifolds $M_{1,2}^{\text {st }}$ of two symmetric saddles $S_{1}$ and $S_{2}$, lead to the conclusion that in $\mathcal{A}_{\text {hidden }}$ a hidden strange attractor is computed.

Also, hidden attractors were computed in modified Chua systems with smooth nonlinearity $\tanh (\cdot)$ [Leonov et al., 2012a] and with nonlinearity $\operatorname{sign}(\cdot)$ [Kuznetsov et al., 2013]. The described approach can be essentially used for investigating other modifications of Chua circuit (see, e.g. [Shi et al., 2008; Banerjee, 2012], etc.).

\section{Conclusions}

In this survey, the notion of hidden attractor, suggested by the authors, is considered. Its connection with well-known fundamental problems and applied models has been demonstrated. New effective analytical and numerical methods for the study of hidden periodic oscillations and hidden chaotic attractors are discussed. The approach, suggested by the authors, are based on the use of modern computers, the development of numerical methods, and the applied bifurcation theory.

At the present time, for the terms hidden oscillations and hidden attractors, Internet searcher system Google gives about 1000 references, including http://en.wikipedia.org/wiki/Hidden_oscillation.

\section{Acknowledgments}

This work was supported by the Academy of Finland, Russian Ministry of Education and Science

\footnotetext{
${ }^{3}$ Lyapunov exponents (LEs) were introduced by Lyapunov [1892] for the analysis of stability by the first approximation for regular time-varying linearizations, where negativeness of the largest Lyapunov exponent indicates stability. In 1948 Chetaev proved that for time-varying linearizations, positive Lyapunov exponent indicates instability in the sense of Lyapunov, but a gap in his proof was found and filled recently for more weak definition of instability [Leonov \& Kuznetsov, 2007b]. While there is no general method for checking regularity of linearization and there are known Perron effects [Leonov \& Kuznetsov, 2007b] of the largest Lyapunov exponent sign inversions for nonregular time-varying linearizations, the computation of Lyapunov exponents for linearization of nonlinear autonomous system along nonstationary trajectories is widely used for the investigation of chaos, where the positiveness of the largest Lyapunov exponent is often considered as indication of chaotic behavior in considered nonlinear system.
} 
(Federal target program), Russian Foundation for Basic Research and Saint-Petersburg State University.

\section{References}

Aizerman, M. \& Gantmacher, F. [1964] Absolute Stability of Regulator Systems (Holden-Day, San Francisco).

Aizerman, M. A. [1949] "On a problem concerning the stability in the large of dynamical systems," Uspekhi Mat. Nauk 4, 187-188 (in Russian).

Aizerman, M. A. [1953] "Physical foundations of the use of small-parameter methods to solve non-linear problems in automatic control theory," Avtomatika $i$ Telemekhanika 14, 597-603 (in Russian).

Albarakati, W. A., Lloyd, N. G. \& Pearson, J. M. [2000] "Transformation to Lienard form," Electron. J. Diff. Eq. 2000, 1-11.

Allgower, E. L. \& Georg, K. [1990] Numerical Continuation Methods: An Introduction (Springer-Verlag, NY).

Alli-Oke, R., Carrasco, J., Heath, W. \& Lanzon, A. [2012] "A robust Kalman conjecture for first-order plants," Preprints of the 7th IFAC Symp. Robust Control Design, pp. 27-32.

Andronov, A. \& Maier, A. [1947] "On the Vyshnegradkii problem in the theory of direct control," Avtomatika $i$ Telemekhanika 8 (in Russian).

Andronov, A. A., Vitt, A. A. \& Khaikin, S. E. [1966] Theory of Oscillators (Pergamon, Oxford).

Aracil, J. \& Gordillo, F. [2004] "Describing function method for stability analysis of PD and PI fuzzy controllers," Fuzzy Sets Syst. 143, 233-249.

Arnold, V. I. [2005] Experimental Mathematics [in Russian] (Fazis, Moscow).

Artes, J. C. \& Llibre, J. [1997] "Quadratic vector fields with a weak focus of third order," Publ. Math. 41, 7-39.

Artes, J. C., Llibre, J. \& Schlomiuk, D. [2006] "The geometry of quadratic differential systems with a weak focus of second order," Int. J. Bifurcation and Chaos 16, 3127-3194.

Artes, J. C., Llibre, J. \& Vulpe, N. [2008] "Singular points of quadratic systems: A complete classification in the coefficient space $R^{12}$," Int. J. Bifurcation and Chaos 18, 313-362.

Bamon, R. [1985] "The solution of Dulac's problem for quadratic vector fields," Ann. Acad. Bris. Cienc., 111-142.

Banerjee, T. [2012] "Single amplifier biquad based inductor-free Chua's circuit," Nonlin. Dyn. 68, 565573.

Barabanov, N. E. [1982] "Meetings of the Leningrad mathematical society (page 164, "Existence of periodic orbits in nonlinear control systems and the
Kalman problem")," Uspekhi Mat. Nauk 37, 163-167 (in Russian).

Barabanov, N. E. [1988] "On the Kalman problem," Sib. Math. J. 29, 333-341.

Bautin, N. N. [1939] "On the number of limit cycles generated on varying the coefficients from a focus or centre type equilibrium state," Doklady Akademii Nauk SSSR 24, 668-671 (in Russian).

Bautin, N. N. [1949] The Behaviour of Dynamical Systems Close to the Boundaries of a Stability Domain (Gostekhizdat, Leningrad, Moscow) [in Russian].

Bautin, N. N. [1952] "On the number of limit cycles appearing on varying the coefficients from a focus or centre type of equilibrium state," Mat. Sb. (N.S.) 30, 181-196 (in Russian).

Bautin, N. N. \& Leontovich, Y. A. [1976] Methods and Procedures for the Qualitative Investigation of Dynamical Systems in a Plane (Nauka, Moscow) [in Russian].

Bellescize, H. [1932] "La réception synchrone," L'onde Électrique 11, 230-340.

Belousov, B. P. [1959] "A periodic reaction and its mechanism," Collection of Short Papers on Radiation Medicine for 1958 (Moscow: Med. Publ.) [in Russian].

Belyustina, L., Bykov, V., Kiveleva, K. \& Shalfeev, V. [1970] "On the size of pull-in range of PLL with proportional-integrating filter," Izv. Vuzov. Radiofizika 13 (in Russian).

Bergen, A. R. \& Franks, R. L. [1971] "Justification of the describing function method," SIAM J. Contr. 9, 568-589.

Bernat, J. \& Llibre, J. [1996] "Counterexample to Kalman and Markus-Yamabe conjectures in dimension larger than 3," Dyn. Contin. Discr. Impul. Syst. 2, 337-379.

Beyn, W. J., Champneys, A., Doedel, E. J., Govaerts, W., Kuznetsov, Y. A. \& Sandstede, B. [2002] "Numerical continuation and computation of normal forms," Handbook of Dynamical Systems, Vol. 2 (Elsevier Science), pp. 149-219.

Bilotta, E. \& Pantano, P. [2008] A Gallery of Chua Attractors, Series A, Vol. 61 (World Scientific).

Blondel, V. \& Megretski, A. [2004] Unsolved Problems in Mathematical Systems and Control Theory (Princeton University Press).

Bogolyubov, N. N. \& Mitropolskii, Y. A. [1961] Asymptotic Methods in the Theory of Non-Linear Oscillations (Gordon and Breach, NY).

Borodzik, M. \& Żołądek, H. [2008] "Small amplitude limit cycles for the polynomial Lienard system," J. Diff. Eqs. 245, 2522-2533.

Bragin, V. O., Vagaitsev, V. I., Kuznetsov, N. V. \& Leonov, G. A. [2011] Algorithms for finding hidden 
oscillations in nonlinear systems. The Aizerman and Kalman conjectures and Chua's circuits," J. Comput. Syst. Sci. Int. 50, 511-543.

Bulgakov, B. V. [1943] "Self-excited oscillations of control systems," Prikl. Mat. Mekh. 7, 97-108 (in Russian).

Bulgakov, B. V. [1954] Oscillations (Gostekhizdat, Moscow) [in Russian].

Cesari, L. [1959] Asymptotic Behavior and Stability Problems in Ordinary Differential Equations (Springer, Berlin).

Chance, B., Pye, E. K., Ghosh, A. K. \& Hess, B. [1973] Biological and Biochemical Oscillators (Academic Press, NY).

Chavarriga, J. \& Grau, M. [2003] "Some open problems related to 16th Hilbert problem," Sci. Ser. A Math. Sci. (N.S.) 9, 1-26.

Chen, G. \& Ueta, T. [1999] "Yet another chaotic attractor," Int. J. Bifurcation and Chaos 9, 1465-1466.

Chen, L. S. \& Wang, M. S. [1979] "The relative position and number of limit cycles of the quadratic differential systems," Acta Math. Sin. 22, 751-758.

Chen, Y., Liu, J. \& Meng, G. [2012] "An incremental method for limit cycle oscillations of an airfoil with an external store," Int. J. Non-Lin. Mech. 47, 75-83.

Cherkas, L. A. \& Zhilevich, L. I. [1970] "Some indications of the lack of uniqueness of limit cycles," Diff. Uravn. 6, 891-897 (in Russian).

Cherkas, L. A. [1986] "Absence of limit cycles around a triple focus in a quadratic system in a plane," Diff. Uravn. 22, 2015-2017 (in Russian).

Christopher, C. \& Li, C. [2007] Limit Cycles of Differential Equations, Advanced Courses in Mathematics (Birkhauser-Verlag, CRM Barcelona, Basel).

Chua, L. \& Lin, G. [1990] "Canonical realization of Chua's circuit family," IEEE Trans. Circuits Syst. 37, 885-902.

Chua, L. [1992] "A zoo of strange attractors from the canonical Chua's circuits," Proc. IEEE 35th Midwest Symp. Circuits and Systems (Cat. No. 92CH3099-9) 2, 916-926.

Cima, A., van den Essen, A., Gasull, A., Hubbers, E. \& Mañosas, F. [1997] "A polynomial counterexample to the Markus-Yamabe conjecture," Adv. Math. 131, 453-457.

Collera, B. \& Chamara, P. [2004] "Structural nonlinearities and the nature of the classic flutter instability," J. Sound Vibr. 277, 711-739.

Coppel, W. A. [1989] "Some quadratic systems with at most one limit cycle. Dynamical report," Expositions Dynamical System (N.S.), pp. 61-88.

Coppel, W. A. [1991] "A new class of quadratic systems," J. Diff. Eqn. 92, 360-372.

de Bruin, J., Doris, A., van de Wouw, N., Heemels, W. \& Nijmeijer, H. [2009] "Control of mechanical motion systems with non-collocation of actuation and friction: A Popov criterion approach for input-to-state stability and set-valued nonlinearities," Automatica 45, 405-415.

de Maesschalck, P. \& Dumortier, F. [2011] "Classical Lienard equations of degree $n \geq 6$ can have $\left[\frac{n-1}{2}+2\right]$ limit cycles," J. Diff. Eqs. 245, 2126-2176.

Doedel, E. [1986] AUTO: Software for Continuation and Bifurcation Problems in Ordinary Differential Equations (California Institute of Technology).

Dulac, H. [1923] "Sur les cycles limites," Bull. Soc. Math. France 51, 45-188.

Dumortier, F., Llibre, J. \& Artes, J. [2006] Qualitative Theory of Planar Differential Systems (Springer, NY).

Earnshaw, R. \& Wiseman, N. [1992] An Introductory Guide to Scientific Visualization (Springer-Verlag).

Ecalle, J. [1992] Introduction aux Functions Analysables et Preuve Constructive de la Conjecture de Dulac (Hermann Publ., Paris).

Efendiev, A. \& Balitinov, M. [1968] "On asymptotic stability in large of a nonlinear system," Diff. Uravneniya 4, 618 (in Russian).

Erugin, N. P. [1952] "A problem in the theory of stability of automatic control systems," Prikl. Mat. Mekh. (in Russian), pp. 620-628.

Feng, L. \& Yirong, L. [2012] "Classification of the centers and isochronicity for a class of quartic polynomial differential systems," Commun. Nonlin. Sci. Numer. Simul. 17, 2270-2291.

Feßler, R. [1995] "A proof of the two-dimensional Markus-Yamabe stability conjecture and a generalization," Ann. Polon. Math. 62, 45-47.

Filippov, A. F. [1988] Differential Equations with Discontinuous Right-Hand Sides (Kluwer, Dordrecht).

Fitts, R. E. [1966] "Two counterexamples to Aizerman's conjecture," Trans. IEEE AC-11, 553-556.

Frommer, M. [1928] "Die integralkurven einer gewonlichen differentialgleichungen erster ordnung in der umgebung rationaler unbestimmtheitsstellen," Quelle: Mathematische Annalen 99, 222-272.

Garber, Y. D. \& Rozenvasser, Y. N. [1965] "The investigation of the periodic modes of non-linear systems using the filter hypothesis," Avtomatika $i$ Telemekhanika 26, 277-287 (in Russian).

Gardner, F. [1966] Phase-Lock Techniques (John Wiley, NY).

Gasull, A., Guillamon, A. \& Manosa, V. [1997] "An explicit expression of the first Liapunov and period constants with applications," J. Math. Anal. Appl. 211, 190-212.

Giné, J. \& Santallusia, X. [2004] "Implementation of a new algorithm of computation of the PoincareLiapunov constants," J. Comput. Appl. Math. 166, 465-476. 
Giné, J. [2007] "On some problems in planar differential systems and Hilbert's 16th problem," Chaos Solit. Fract. 31, 1118-1134.

Giné, J. [2009] "On the number of algebraically independent Poincare-Liapunov constants," Appl. Math. Comput. 188, 1870-1877.

Giné, J. [2012] "Limit cycle bifurcations from a nondegenerate center," Appl. Math. Comput. 218, 47034709 .

Glutsyuk, A. A. [1995] "Asymptotic stability of linearizations of a planar vector field with a singular point implies global stability," Funct. Anal. Its Appl. 29, 238-247.

Glutsyuk, A. A. [1998] "Meetings of the Moscow mathematical society (1997)," Russ. Math. Surv. 53, 413417.

Grabowski, P. [2011] "Absolute stability criteria for infinite dimensional discrete lur'e systems with application to loaded distortionless electric RLCGtransmission line," J. Diff. Eqs. Appl., doi:10.1080/ 10236198.2011.639366.

Gubar', N. A. [1961] "Investigation of a piecewise linear dynamical system with three parameters," J. Appl. Math. Mech. 25, 1011-1023.

Gutierrez, C. [1995] "A solution to the bidimensional global asymptotic stability conjecture," Ann. Inst. H. Poincaré Anal. Non Lineaire. 12, 627-671.

Hairer, E. \& Wanner, G. [1991] Solving Ordinary Differential Equations II (Stiff and Differential-Algebraic Problems) (Springer-Verlag, Berlin).

Han, M. \& Li, J. [2012] "Lowerbounds for the Hilbert number of polynomial systems," J. Diff. Eqs. 252, 3278-3304.

Hilbert, D. [1901-1902] "Mathematical problems," Bull. Amer. Math. Soc., 437-479.

Hsu, J. C. \& Meyer, A. U. [1968] Modern Control Principles and Applications (McGraw-Hill).

Huang, J., Wang, F., Wang, L. \& Yang, J. [2008] "A quartic system and a quintic system with fine focus of order 18," Bull. Sci. Math. 132, 205-217.

Ilyashenko, Y. [1985] "Dulac's memoir 'On limit cycles' and related problems of the local theory of differential equations," Russ. Math. Surv. 40, 1-49.

Ilyashenko, Y. [1991] Finiteness for Limit Cycles (Amer. Math. Soc., Providence, RI).

Jones, D. S., Plank, M. J. \& Sleeman, B. D. [2010] Differential Equations and Mathematical Biology (CRC Press, London).

Kalman, R. E. [1957] "Physical and mathematical mechanisms of instability in nonlinear automatic control systems," Trans. ASME 79, 553-566.

Kapranov, M. [1956] "Locking band for phase-locked loop," Radiofizika 2, 37-52 (in Russian).

Khalil, H. K. [2002] Nonlinear Systems (Prentice Hall, NJ).
Kiseleva, M. A., Kuznetsov, N. V., Leonov, G. A. \& Neittaanmäki, P. [2012] "Drilling systems failures and hidden oscillations," NSC 2012 — 4th IEEE Int. Conf. Nonlinear Science and Complexity, pp. 109112.

Krasovsky, N. N. [1952] "Theorems on the stability of motions determined by a system of two equations," Prikl. Mat. Mekh. 16, 547-554 (in Russian).

Krauskopf, B., Osinga, H. M. \& Galan-Vioque, J. E. [2007] Numerical Continuation Methods for Dynamical Systems (Springer, Dordrecht, The Netherlands).

Krylov, A. N. [1936] The Vibration of Ships (GRSL, Moscow) [in Russian].

Krylov, N. \& Bogolyubov, N. [1947] Introduction to NonLinear Mechanics (Princeton Univ. Press, Princeton).

Kudrewicz, J. \& Wasowicz, S. [2007] Equations of PhaseLocked Loop. Dynamics on Circle, Torus and Cylinder, A, Vol. 59 (World Scientific).

Kuznetsov, N. V. [2008] Stability and Oscillations of Dynamical Systems: Theory and Applications (Jyvaskyla University Printing House).

Kuznetsov, N. V. \& Leonov, G. A. [2008a] "Computation of Lyapunov quantities," 6th EUROMECH Nonlinear Dynamics Conf. (http://lib.physcon.ru/?item=1802).

Kuznetsov, N. V. \& Leonov, G. A. [2008b] "Lyapunov quantities, limit cycles and strange behavior of trajectories in two-dimensional quadratic systems," J. Vibroengin. 10, 460-467.

Kuznetsov, N. V., Leonov, G. A. \& Vagaitsev, V. I. [2010] "Analytical-numerical method for attractor localization of generalized Chua's system," IFAC Proc. Vol. (IFAC-PapersOnline) 4, pp. 29-33.

Kuznetsov, N. V., Kuznetsova, O. A., Leonov, G. A. \& Vagaytsev, V. I. [2011a] "Hidden attractor in Chua's circuits," ICINCO 2011 - Proc. 8th Int. Conf. Informatics in Control, Automation and Robotics, pp. 279283.

Kuznetsov, N. V., Leonov, G. A. \& Seledzhi, S. M. [2011b] "Hidden oscillations in nonlinear control systems," IFAC Proc. Vol. (IFAC-PapersOnline) 18, $2506-2510$.

Kuznetsov, N. V., Leonov, G. A., Yuldashev, M. V. \& Yuldashev, R. V. [2011c] "Analytical methods for computation of phase-detector characteristics and PLL design," ISSCS 2011 - Int. Symp. Signals, Circuits and Systems, Proc., pp. 7-10.

Kuznetsov, N. V., Kuznetsova, O. A. \& Leonov, G. A. [2013] "Visualization of four normal size limit cycles in two-dimensional polynomial quadratic system," Diff. Eqs. Dyn. Syst. 21, 29-34.

Kuznetsov, N., Kuznetsova, O., Leonov, G. \& Vagaitsev, V. [2013] "Analytical-numerical localization of hidden attractor in electrical Chua's circuit," Informatics in Control, Automation and Robotics, Lecture Notes in 
Electrical Engineering, Vol. 174, Part 4 (Springer), pp. 149-158.

LaBryer, A. \& Attar, P. [2010] "A harmonic balance approach for large-scale problems in nonlinear structural dynamics," Comput. Struct. 88, 1002-1014.

Lauvdal, T., Murray, R. \& Fossen, T. [1997] "Stabilization of integrator chains in the presence of magnitude and rate saturations: A gain scheduling approach," Proc. IEEE Control and Decision Conf., pp. 44044005.

Lefschetz, S. [1957] Differential Equations: Geometric Theory (Interscience, NY-London).

Lefschetz, S. L. [1965] Stability of Nonlinear Control Systems (Academic Press, NY-London).

Leonov, G. A. [1970] "On the necessity for the frequency condition of absolute stability of time-independent systems in the critical case of a pair of imaginary roots," Doklady Akademii Nauk SSSR 193, 756-759 (in Russian).

Leonov, G. A. [1981] "The stability in large of nonlinear systems in the critical case of two zero roots," Prikl. Mat. Mekh. 45, 752-755 (in Russian).

Leonov, G. A., Ponomareko, D. V. \& Smirnova, V. B. [1996] Frequency-Domain Methods for Nonlinear Analysis. Theory and Applications (World Scientific, Singapore).

Leonov, G. A. [1997] "Two-dimensional quadratic systems as a Lienard equation," Diff. Eqs. Dyn. Syst. 5, 289-297.

Leonov, G. A. [1998] "The problem of estimation of the number of cycles of two-dimensional quadratic systems from nonlinear mechanics point of view," Ukr. Math. J. 50, 48-57.

Leonov, G. A. [2006] "Families of transverse curves for two-dimensional systems of differential equations," Vestnik St. Petersburg University, pp. 48-78.

Leonov, G. A. \& Kuznetsov, N. V. [2007a] "Computation of the first Lyapunov quantity for the second-order dynamical system," IFAC Proc. Vol. (IFAC-PapersOnline) 3, 87-89.

Leonov, G. A. \& Kuznetsov, N. V. [2007b] "Time-varying linearization and the Perron effects," Int. J. Bifurcation and Chaos 17, 1079-1107.

Leonov, G. A., Kuznetsov, N. V. \& Kudryashova, E. V. [2011a] "Cycles of two-dimensional systems. Computer calculations, proofs, and experiments," Vestnik St. Petersburg Univ. Mathematics 41, 216-250.

Leonov, G. A. [2008a] "Hilbert's 16th problem for quadratic system. New method based on a transformation to the Lienard equation," Int. J. Bifurcation and Chaos 18, 877-884.

Leonov, G. A. [2008b] Strange Attractors and Classical Stability Theory (St. Petersburg University Press, St. Petersburg).
Leonov, G. A. \& Kuznetsova, O. A. [2009] "Evaluation of the first five Lyapunov exponents for the Lienard system," Dokl. Phys. 54, 131-133.

Leonov, G. A., Kuznetsov, N. V. \& Seledzhi, S. M. [2009] "Nonlinear analysis and design of phase-locked loops," Automation Control - Theory and Practice (In-Tech), pp. 89-114.

Leonov, G. A. [2009a] "Limit cycles of the Lienard equation with discontinuous coefficients," Dokl. Phys. 54, 238-241.

Leonov, G. A. [2009b] "On Aizerman problem," Automat. Remote Contr. 7, 37-49.

Leonov, G. A. [2009c] "On the harmonic linearization method," Dokl. Math. 79, 144-146.

Leonov, G. A. [2009d] "On the method of harmonic linearization," Automat. Remote Contr. 70, 800810.

Leonov, G. A. \& Kuznetsova, O. A. [2010] "Lyapunov quantities and limit cycles of two-dimensional dynamical systems. Analytical methods and symbolic computation," Regul. Chaot. Dyn. 15, 354-377.

Leonov, G. A. \& Kuznetsov, N. V. [2010] "Limit cycles of quadratic systems with a perturbed weak focus of order 3 and a saddle equilibrium at infinity," Dokl. Math. 82, 693-696.

Leonov, G. A. [2010a] "Effective methods for periodic oscillations search in dynamical systems," Appl. Math. Mech. 74, 24-50.

Leonov, G. A. [2010b] "Necessary and sufficient conditions for the boundedness of solutions to two-dimensional quadratic systems in a positively invariant half-plane," Dokl. Math. 81, 31-33.

Leonov, G. A., Bragin, V. O. \& Kuznetsov, N. V. [2010a] "Algorithm for constructing counterexamples to the Kalman problem," Dokl. Math. 82, 540-542.

Leonov, G. A., Bragin, V. O. \& Kuznetsov, N. V. [2010b] "On problems of Aizerman and Kalman," Vestnik St. Petersburg Univ. Mathematics 43, 148-162.

Leonov, G. A., Vagaitsev, V. I. \& Kuznetsov, N. V. [2010c] "Algorithm for localizing Chua attractors based on the harmonic linearization method," Dokl. Math. 82, 693-696.

Leonov, G. A. [2011] "Four normal size limit cycles in two-dimensional quadratic systems," Int. J. Bifurcation and Chaos 21, 425-429.

Leonov, G. A. \& Kuznetsov, N. V. [2011a] "Algorithms for searching for hidden oscillations in the Aizerman and Kalman problems," Dokl. Math. 84, 475-481.

Leonov, G. A. \& Kuznetsov, N. V. [2011b] "Analyticalnumerical methods for investigation of hidden oscillations in nonlinear control systems," IFAC Proc. Vol. (IFAC-PapersOnline) 18, 2494-2505.

Leonov, G. A., Kuznetsov, N. V. \& Kudryashova, E. V. [2011a] "A direct method for calculating Lyapunov 
quantities of two-dimensional dynamical systems," Proc. Steklov Instit. Math. 272(Suppl. 1), S119-S127.

Leonov, G. A., Kuznetsov, N. V., Kuznetsova, O. A., Seledzhi, S. M. \& Vagaitsev, V. I. [2011b] "Hidden oscillations in dynamical systems," Trans. Syst. Contr. 6, 54-67.

Leonov, G. A., Kuznetsov, N. V. \& Vagaitsev, V. I. [2011c] "Localization of hidden Chua's attractors," Phys. Lett. A 375, 2230-2233.

Leonov, G. A., Kuznetsov, N. V., Yuldahsev, M. V. \& Yuldashev, R. V. [2011d] "Computation of phase detector characteristics in synchronization systems," Dokl. Math. 84, 586-590.

Leonov, G. A., Kuznetsov, N. V. \& Vagaitsev, V. I. [2012a] "Hidden attractor in smooth Chua systems," Physica D 241, 1482-1486.

Leonov, G. A., Kuznetsov, N. V., Yuldahsev, M. V. \& Yuldashev, R. V. [2012b] "Analytical method for computation of phase-detector characteristic," IEEE Trans. Circuits Syst.-II: Express Briefs 59, 633-647.

Leonov, G. \& Kuznetsov, N. V. [2013a] "Analyticalnumerical methods for hidden attractors' localization: The 16th Hilbert problem, Aizerman and Kalman conjectures, and Chua circuits," Numerical Methods for Differential Equations, Optimization, and Technological Problems, Computational Methods in Applied Sciences, Vol. 27, Part 1 (Springer), pp. 41-64.

Leonov, G. A. \& Kuznetsov, N. V. [2013b] "Hidden oscillations in dynamical systems: 16 Hilbert's problem, Aizerman's and Kalman's conjectures, hidden attractors in Chua's circuits," J. Math. Sci. [in print].

Li, C. [1986] "Non-existence of limit cycle around a weak focus of order three for any quadratic system," Chin. Ann. Math. 7B, 174-190.

Li, J. [2003] "Hilbert's 16-th problem and bifurcations of planar polynomial vector fields," Int. J. Bifurcation and Chaos 13, 47-106.

Li, J., Chen, Y., Zhang, W. \& Tian, Y. [2008] "Computation of Lyapunov values for two planar polynomial differential systems," Appl. Math. Comput. 204, 240248.

Li, C., Liu, C. \& Yanga, J. [2009] "A cubic system with thirteen limit cycles," J. Diff. Eqs. 246, 3609-3619.

Li, H., Wu, Y. \& Jin, Y. [2012] "Local bifurcation of limit cycles and integrability of a class of nilpotent systems," Adv. Diff. Eqs. 20, doi:10.1186/1687-18472012-20.

Liberzon, M. R. [2006] "Essays on the absolute stability theory," Automat. Rem. Contr. 67, 1610-1644.

Lindsey, W. [1972] Synchronization Systems in Communication and Control (Prentice-Hall, NJ).

Liu, L., Thomas, J., Dowell, E., Attar, P. \& Hall, K. [2006] "An incremental method for limit cycle oscillations of an airfoil with an external store," J. Comput. Phys. 215, 298-320.
Llibre, J., Ponce, E. \& Ros, J. [2011] "Algebraic determination of limit cycles in a family of three-dimensional piecewise linear differential systems," Nonlin. Anal. 74, 6712-6727.

Lloyd, N. G. [1988] "Limit cycles of polynomial systems - some recent developments," New Direction in Dynamical Systems (Cambridge Univ. Press), pp. $192-234$.

Lorenz, E. N. [1963] "Deterministic nonperiodic flow," J. Atmos. Sci. 20, 130-141.

Lyapunov, A. M. [1892] The General Problem of the Stability of Motion (Kharkov).

Lynch, S. [2010] Dynamical Systems with Applications using Maple (Birkhäuser, Springer, Boston).

Malkin, I. G. [1952] "On the stability of automatic control systems," Prikl. Mat. Mekh., 495-499 (in Russian).

Margaliota, M. \& Yfoulis, C. [2006] "Absolute stability of third-order systems: A numerical algorithm," Automatica 42, 1705-1711.

Margaris, W. [2004] Theory of the Non-Linear Analog Phase Locked Loop (Springer-Verlag, NJ).

Markus, L. \& Yamabe, H. [1960] "Global stability criteria for differential systems," Osaka Math. J. 12, 305317.

Marsden, J. \& McCracken, M. [1976] Hopf Bifurcation and Its Applications (Springer, NY).

Matsumoto, T. [1990] "A chaotic attractor from Chua's circuit," IEEE Trans. Circuits Syst. 31, 1055-1058.

Mees, A. I. \& Bergen, A. R. [1975] "Describing functions revisited," IEEE Trans. Automat. Contr. AC20, 473-478.

Meisters, G. [1996] "A biography of the Markus-Yamabe conjecture," http://www.math.unl.edu/ gmeisters1/ papers/HK1996.pdf.

Migulin, V. V., Medvedev, V. I., Mustel, Y. R. \& Parygin, V. N. [1978] Foundations of the Oscillation Theory (Nauka, Moscow) (in Russian).

Mihajlovic, N., van Veggel, A., van de Wouw, N. \& Nijmeijer, H. [2004] "Analysis of friction-induced limit cycling in an experimental drill-string system," $J$. Dyn. Syst. Meas. Contr. 126, 709-720.

Noldus, E. [1971] "A counterpart of Popov's theorem for the existence of periodic solutions," Int. J. Contr. 13, 705-719.

Pearson, J. \& Lloyd, N. [2009] "Kukles revisited: Advances in computing techniques," Comput. Math. Appl. 60, 2797-2805.

Petrovskii, I. G. \& Landis, Y. M. [1955] "On the number of limit cycles of the equation $\frac{d y}{d x}=\frac{P(x, y)}{Q(x, y)}$, where $P$ and $Q$ are 2-nd degree polynomials," Mat. Sb. (N.S.) 37, 209-250.

Petrovskii, I. G. \& Landis, Y. M. [1959] "Corrections to the papers on the number of limit cycles of the 
equation $\frac{d y}{d x}=\frac{P(x, y)}{Q(x, y)}$, where $P$ and $Q$ are 2-nd degree polynomials and on the number of limiting cycles of the equation $\frac{d y}{d x}=\frac{P(x, y)}{Q(x, y)}$, where $P$ and $Q$ are polynomials," Mat. Sb. (N.S.) 48, 253-255.

Pliss, V. A. [1958] Some Problems in the Theory of the Stability of Motion (Izd LGU, Leningrad) [in Russian].

Poincaré, H. [1881] "Mémoire sur les courbes définies par les équations différentielles," J. de Math. 37, 375-422.

Poincaré, H. [1885] "Memoire sur les courbes definies par lesequations diffeentielles," J. de. Math. Pures et Appliquees, 167-244.

Qiu, Y. \& Yang, J. [2009] "On the focus order of planar polynomial differential equations," J. Diff. Eqs. 246, 3361-3379.

Rasvan, V. [2004] "Delay independent and delay dependent Aizerman," Unsolved Problems in Mathematical Systems and Control Theory, eds. Blondel, V. D. \& Megretski, A. (Princeton University Press), pp. 212220.

Rayleigh, J. W. S. [1877] The Theory of Sound (MacMillan, London).

Reyn, J. W. [1994] A Bibliography of the Qualitative Theory of Quadratic Systems of Differential Equations in the Plane. 3rd edition (Report 94-02. Delft University of Technology).

Roussarie, R. [1998] Bifurcations of Planar Vector Fields and Hilbert's Sixteenth Problem, Progress in Mathematics, Vol. 164 (Birkhauser, Basel-Boston-Berlin).

Sabatini, M. \& Chavarriga, J. [1999] "A survey of isochronous centers," Qualit. Th. Dyn. Syst. 1, $1-70$.

Schlomiuk, D. \& Pal, J. [2001] "The geometry in the neighborhood of infinity of quadratic differential systems with a weak focus," Qualit. Th. Dyn. Syst. 2, $1-43$.

Schlomiuk, D. \& Vulpe, N. [2005] "Geometry of quadratic differential systems in the neighborhood of infinity," J. Diff. Eqs. 215, 357-400.

Schuko, S. D. [1968] "Lyapunov quantities computation with the help of EVZM," Trudy Gor'kovskogo Inst. Inzh. Vodn. Transp. 94, 97-109 (in Russian).

Serebryakova, N. [1959] "Behavior of dynamical system with one degree of freedom near the points of boundaries of domain of stability in the case when a safe boundary goes to the dangerous one," Izvestiya AN SSSR. Division of Technical Sciences, Mechanics and Engineering, pp. 178-182 (in Russian).

Shafer, S. [2009] "Symbolic computation and the cyclicity problem for singularities," J. Symbol. Comput. 47, 1140-1153.

Shakhgil'dyan, V. \& Lyakhovkin, A. [1972] Sistemy Fazovoi Avtopodstroiki Chastoty (Phase Locked Systems) (Svyaz', Moscow) [in Russian].
Shi, S. [1980] "A concrete example of the existence of four limit cycles for plane quadratic systems," Scientific Sinica 23, 153-158.

Shi, Z., Hong, S. \& Chen, K. [2008] "Experimental study on tracking the state of analog Chua's circuit with particle filter for chaos synchronization," Phys. Lett. A 372, 5575-5580.

Smale, S. [1998] "Mathematical problems for the next century," The Math. Intell. 20, 7-15.

Stoker, J. J. [1950] Nonlinear Vibrations in Mechanical and Electrical Systems (Interscience, N.Y: L.).

Strogatz, H. [1994] Nonlinear Dynamics and Chaos. With Applications to Physics, Biology, Chemistry, and Engineering (Westview Press).

Suarez, A. \& Quere, R. [2003] Stability Analysis of Nonlinear Microwave Circuits (Artech House, NJ).

Tesi, A., Abed, E., Genesio, R. \& Wang, H. [1996] "Harmonic balance analysis of period-doubling bifurcations with implications for control of nonlinear dynamics," Automatica 32, 1255-1271.

Timoshenko, S. [1928] Vibration Problems in Engineering (Van Nostrand, NY).

Tricomi, F. [1933] "Integrazione di unequazione differenziale presentatasi in elettrotechnica," Annali della $R$. Shcuola Normale Superiore di Pisa 2, 1-20.

Tzipkin, Y. Z. [1984] Relay Control Systems (Cambridge Univ Press., Cambridge).

Ueda, Y., Akamatsu, N. \& Hayashi, C. [1973] "Computer simulations and non-periodic oscillations," Trans. IEICE Japan 56A, 218-255.

van der Pol, B. [1926] "On relaxation-oscillations," Philos. Mag. J. Sci. 7, 978-992.

Viterbi, A. [1966] Principles of Coherent Communications (McGraw-Hill, NY).

Wendt, K. \& Fredentall, G. [1943] "Automatic frequency and phase control of synchronization in TV receivers," Proc. IRE 31, 1-15.

Westphal, L. C. [2001] Handbook of Control Systems Engineering (Springer).

Yakobovich, V., Leonov, G. A. \& Gelig, A. [2004] Stability of Stationary Sets in Control Systems with Discontinuous Nonlinearities (World Scientific, Singapore).

Yakubovich, V. A. [1958] "On boundedness and stability in large of solutions of some nonlinear differential equations," Doklady Akademii Nauk SSSR 121, 984986 (in Russian).

Yang, J. \& Han, M. [2012] "Computation of expansion coefficients of Melnikov functions near a nilpotent center," Computers and Mathematics with Applications, in press.

Ye, Y.-Q. et al. [1986] Theory of Limit Cycles (Amer. Math. Soc., Providence, RI).

$\mathrm{Yu}, \mathrm{P}$. [1998] "Computation of normal forms via a perturbation technique," J. Sound Vibr. 211, 19-38. 
Yu, P. \& Chen, G. [2008] "Computation of focus values with applications," Nonlin. Dyn. 51, 409-427.

Yu, P. \& Corless, R. [2009] "Symbolic computation of limit cycles associated with Hilberts 16th problem," Commun. Nonlin. Sci. Numer. Simul. 14, 4041-4056.

Zhang, P. [2002] "On the distribution and number of limit cycles for quadratic systems with two foci," Qual. Th. Dyn. Syst. 3, 437-463.
Zhou, J. \& Zhang, L. [2005] "Incremental harmonic balance method for predicting amplitudes of a multid.o.f. non-linear wheel shimmy system with combined Coulomb and quadratic damping," J. Sound Vibr. 279, 403-416.

Żołądek, H. [1995] "Eleven small limit cycles in a cubic vector field," Nonlinearity 8, 843-860. 